\title{
Long slit spectroscopy of a sample of isolated spirals with and without an $\mathrm{AGN}^{\star, \star \star, \star \star \star}$
}

\author{
I. Márquez ${ }^{1}$, F. Durret ${ }^{2}$, J. Masegosa ${ }^{1}$, M. Moles ${ }^{1}$, J. Varela ${ }^{1}$, R. M. González Delgado ${ }^{1}$, J. Maza ${ }^{3}$, \\ E. Pérez ${ }^{1}$, and M. Roth ${ }^{4}$ \\ 1 Instituto de Astrofísica de Andalucía (CSIC), Apartado 3004, 18080 Granada, Spain \\ 2 Institut d'Astrophysique de Paris, CNRS, 98bis Bd Arago, 75014 Paris, France \\ 3 Departamento de Astronomía, Universidad de Chile, Casilla 36D, Santiago, Chile \\ ${ }^{4}$ Observatories of the Carnegie Institution of Washington, 813 Barbara Street, Pasadena, CA91101, USA
}

Received 24 July 2003 / Accepted 18 November 2003

\begin{abstract}
We present the kinematical data obtained for a sample of active (Seyfert) and non active isolated spiral galaxies, based on long slit spectra along several position angles in the $\mathrm{H} \alpha$ line region and, in some cases, in the $\mathrm{Ca}$ triplet region as well. Gas velocity distributions are presented, together with a simple circular rotation model that allows us to determine the kinematical major axes. Stellar velocity distributions are also shown. The main result is that active and control galaxies seem to be equivalent in all kinematical aspects. For both subsamples, the departure from pure circular rotation in some galaxies can be explained by the presence of a bar and/or of a spiral arm. They also present the same kind of peculiarities, in particular, S-shape structures are quite common near the nuclear regions. They define very similar Tully-Fisher relations. Emission line ratios are given for all the detected HII regions; the analysis of the $[\mathrm{NII}] / \mathrm{H} \alpha$ metallicity indicator shows that active and non-active galaxies have indistinguishable disk metallicities. These results argue in favour of active and non-active isolated spiral galaxies having essentially the same properties, in agreement with our previous results based on the analysis of near infrared images. It appears now necessary to confirm these results on a larger sample.
\end{abstract}

Key words. galaxies: spiral - galaxies: kinematics and dynamics - galaxies: structure - galaxies: interactions

\section{Introduction}

The DEGAS collaboration was started a few years ago to search for a sufficient condition for a galaxy to host an active nucleus. The active galaxies have been chosen with the following criteria: (a) catalogued as Seyfert 1 or 2 in Véron-Cetty $\&$ Véron's catalogue (we have checked that they were all classified as Seyferts in the NED database and give their AGN type in Table 1); (b) with morphological information in the RC3 catalogue (de Vaucouleurs et al. 1991); (c) isolated, in the sense of not having a companion within $0.4 \mathrm{Mpc}$

Send offprint requests to: I. Márquez, e-mail: isabel@iaa.es

* Based on observations made with WHT operated on the island of La Palma by ING in the Spanish Observatorio del Roque de Los Muchachos of the Instituto de Astrofísica de Canarias, the European Southern Observatory (La Silla), Calar Alto Observatory (Almería, Spain) and Las Campanas Observatories (Chile).

$\star \star$ Table 3 and Figs. 3, 5, 7, 9, 11, 13, 15, 17, 19, 21, 23, 25, 28, $30,32,34,36,38,40,42,44,46,48,50$ and 52 are only available in electronic form at http://www. edpsciences . org

$\star \star \star$ Table 5 is only available in electronic form at the CDS via anonymous ftp to cdsarc.u-strasbg.fr (130.79.128.5) or via http://cdsweb.u-strasbg.fr/cgi-bin/qcat?]/A+A/416/475
$\left(H_{0}=75 \mathrm{~km} \mathrm{~s}^{-1} \mathrm{Mpc}^{-1}\right)$ and $c z<500 \mathrm{~km} \mathrm{~s}^{-1}$, or companions catalogued by Nilson without known redshift; (d) nearby, $c z<6000 \mathrm{~km} \mathrm{~s}^{-1}$; and (e) intermediate inclination $\left(30\right.$ to $65^{\circ}$ ). Note however that the sample of active galaxies thus defined may not be representative of the whole AGN population, since the selection criteria of the Véron-Cetty \& Véron catalogue are not necessarily complete. The non-active galaxies have been selected among spirals satisfying the same conditions (b), (c), (d) and (e), and with morphologies (given by the complete de Vaucouleurs coding, not just the Hubble type) similar to those of the active spirals. A total of 17 active galaxies and 16 non active galaxies (used as control sample), with similar distribution of morphological types (including the bar type), were selected. Since all galaxies were chosen to be isolated under the criteria defined above, side effects that could be produced by the gravitational interaction with nearby galaxies are avoided. The details of the program and sample definition are given in Márquez et al. $(1999,2000)$, where it has been shown that the non-active galaxies are well suited to be used as a control sample. Basic properties of the galaxies of our sample are given in Table 1. The observations we carried out included infrared and optical imaging and long slit spectroscopy to derive the kinematical properties of the ionized gas and, for 
some of them, of the stellar component. Infrared HST images of our selected galaxies were also retrieved and analyzed when available.

The data already presented have shown some interesting results: the analysis of the infrared images indicated that there are no morphological differences between active and non active galaxies in our sample. We also found that most galaxies have bars and sometimes nuclear bars within the large bars. Some of these inner bars were reported for the first time (Márquez et al. 1999, 2000). The full analysis of NGC 6951, the first galaxy of our sample for which all the required data became available (Pérez et al. 2000), led us to discover the decoupling between the stellar and gas kinematics in the central regions, and to hint the presence of a younger stellar population there. Velocity drops were detected in the nuclear regions of five of our sample galaxies. The analysis of the calcium triplet (CaT) lines equivalent widths indicated the presence of a different stellar population spatially coincident with the kinematical drops. Further analysis of the HST images of a total of 14 galaxies with reported velocity dispersion drops (including our five), show evidence for central elongations in most of them, which could be related with the mechanism invoked in numerical models to reproduce such drops (see Márquez et al. 2003 and references therein).

The present work includes the kinematical data on the gas and stars obtained for the galaxies of our sample, together with the emission line ratios measured in the nuclear and extranuclear HII regions of the same galaxies. The metallicities of these HII regions, estimated from the observed line ratios, are briefly discussed. A general discussion based on the DEGAS data will be presented in a forthcoming paper.

The paper is organized as follows. The observations and data reduction procedures are presented in Sect. 2. Characteristics of the rotation curves for each galaxy, together with a discussion of the positions of our sample galaxies in the Tully-Fisher relation are presented in Sect. 3. The properties of nuclear and extra-nuclear HII regions are described in Sect. 4. The summary and conclusions are presented in Sect. 5 .

\section{Observations and data reduction}

The spectroscopic data were collected with four different instruments: the Boller \& Chivens spectrograph attached to the $1.5 \mathrm{~m}$ ESO telescope at La Silla (Chile), the Modular Spectrograph at the $2.5 \mathrm{~m}$ Dupont telescope at Las Campanas (Chile), the ISIS Spectrograph at the $4.2 \mathrm{~m}$ William Herschel telescope in La Palma (Spain) and the TWIN Spectrograph attached to the $3.5 \mathrm{~m}$ telescope in Calar Alto (CAHA, Spain). The setup and main characteristics of the observations are given in Table 2.

Standard IRAF ${ }^{1}$ procedures were used for the reduction of the spectroscopic data, following the standard steps of bias

${ }^{1}$ IRAF is the Image Analysis and Reduction Facility made available to the astronomical community by the National Optical Astronomy Observatories, which are operated by the Association of Universities for Research in Astronomy (AURA), Inc., under contract with the US National Science Foundation.
Table 1. Main properties of the galaxies.

\begin{tabular}{|c|c|c|c|}
\hline Galaxy & $\begin{array}{l}\text { Type } \\
\text { (RC3) } \\
(1)\end{array}$ & $\begin{array}{l}m_{B} \\
(\mathrm{mag}) \\
(2)\end{array}$ & $\begin{array}{l}\text { AGN } \\
\text { type } \\
\text { (3) }\end{array}$ \\
\hline UGC 1395 & .SAT3.. & 14.18 & 1.9 \\
\hline IC 184 & .SBR1* & 14.66 & 2 \\
\hline IC 1816 & .SBR2P? & 13.83 & 1 \\
\hline UGC 3223 & .SB.1.. & 13.83 & 1 \\
\hline NGC 2639 & RSAR1* & 12.59 & 1.9 \\
\hline IC 2510 & .SBT2* & $14.00^{*}$ & 2 \\
\hline NGC 3660 & .SBR4.. & 12.29 & 2 \\
\hline NGC 4507 & PSXT3.. & 12.78 & 2 \\
\hline NGC 4785 & PSBR3*. & 13.21 & 2 \\
\hline NGC 5347 & PSBT2.. & 13.16 & 2 \\
\hline NGC 5728 & .SXR1* & 12.37 & 2 \\
\hline ESO139-12 & PSAT4P* & 13.59 & 2 \\
\hline NGC 6814 & .SXT4.. & 11.85 & 1.5 \\
\hline NGC 6860 & PSBR3.. & 13.68 & 1 \\
\hline NGC 6890 & .SAT3.. & 13.05 & 2 \\
\hline NGC 6951 & .SXT4.. & 11.91 & 2 \\
\hline NGC 151 & .SBR4.. & 12.23 & \\
\hline NGC 1357 & .SAS2.. & 12.46 & \\
\hline IC 454 & SB.2.. & $14.50^{*}$ & \\
\hline NGC 2712 & .SBR3* & 12.38 & \\
\hline NGC 2811 & .SBT1.. & 12.66 & \\
\hline NGC 3571 & PSXT1* & 12.99 & \\
\hline NGC 3835 & $. S . .2 * /$ & 13.20 & \\
\hline NGC 4162 & RSAT4.. & 12.55 & \\
\hline NGC 4290 & .SBT2* & 12.66 & \\
\hline NGC 6012 & RSBR2*. & 12.69 & \\
\hline NGC 6155 &.$S ? \ldots$ & 13.20 & \\
\hline NGC 7328 & $. S . .2 .$. & 13.98 & \\
\hline
\end{tabular}

(1) Morphological type taken from the RC3 catalogue;

(2) $B$ magnitude as given in the RC3 catalogue;

(3) AGN Seyfert type taken from the NED.

* Taken from NED.

subtraction, flat field correction, wavelength calibration with arc lamps observed before and after the target, atmospheric extinction correction, and flux calibration using spectroscopic standards observed through an 8 arcsec wide slit for WHT runs. The sky background level was determined taking median averages over two strips on either side of the galaxy signal. The parameters of the lines were measured with the program splot. The errors in Table 5 have been calculated by quadratic 
Table 2. Observations.

\begin{tabular}{|c|c|c|c|c|c|c|c|c|c|}
\hline Code & Telescope & Date & Instrument & $\begin{array}{c}\text { Spectral } \\
\text { sampling } \\
(\AA / \text { pix })\end{array}$ & $\begin{array}{c}\text { Spectral } \\
\text { resolution } \\
(\AA ̊)\end{array}$ & $\begin{array}{l}\text { Range } \\
(\AA)\end{array}$ & $\begin{array}{c}\text { Slit } \\
\text { width } \\
(\operatorname{arcsec})\end{array}$ & $\begin{array}{c}\text { Spatial } \\
\text { sampling } \\
\text { (arcsec/pix) }\end{array}$ & $\begin{array}{l}\text { Average } \\
\text { seeing } \\
(\operatorname{arcsec})\end{array}$ \\
\hline ESO 96 & $1.5 \mathrm{~m}$ ESO & Jan. 1996 & Boller \& Chivens & 0.98 & 1.86 & $5493-7505$ & 2 & 0.78 & 1 \\
\hline LC 596 & Dupont & May 1996 & Boller \& Chivens & 1.0 & 1.10 & $6166-7190$ & 1.25 & 0.56 & 1.5 \\
\hline LC 896 & & Aug. 1996 & & 1.0 & 1.29 & $6149-7181$ & 1.2 & 0.67 & \\
\hline LC 898 & & Aug. 1998 & & 0.99 & 1.28 & $5540-7558$ & 1.2 & 0.584 & \\
\hline \multirow[t]{3}{*}{ WHT 96} & WHT & Aug. 1996 & ISIS & 0.39 & 0.81 & $4757-5141$ & 1.03 & 0.36 & 1.2 \\
\hline & & & & $"$ & " & $6493-6880$ & $"$ & " & " \\
\hline & & & & " & " & $8505-8881$ & " & $\prime \prime$ & $\prime \prime$ \\
\hline \multirow[t]{3}{*}{ WHT 99} & & Dec. 1999 & ISIS & 0.44 & 0.92 & $3654-5187$ & 1.03 & 0.20 & 1.5 \\
\hline & & & & $"$ & $"$ & $5354-6894$ & $"$ & $"$ & $"$ \\
\hline & & & & 0.39 & 0.81 & $8476-8872$ & " & 0.36 & " \\
\hline \multirow[t]{3}{*}{ WHT 00} & & May 2000 & ISIS & 0.44 & 0.92 & $3649-5185$ & 1.00 & 0.20 & 1.3 \\
\hline & & & & $"$ & $"$ & $5334-6874$ & $"$ & $"$ & " \\
\hline & & & & 0.39 & 0.81 & $8456-8852$ & " & 0.36 & $"$ \\
\hline \multirow[t]{2}{*}{ CAHA 00} & $3.5 \mathrm{~m}$ CAHA & Jan. 2000 & TWIN & 0.38 & 0.80 & $4600-5380$ & 1.20 & 0.56 & 1.2 \\
\hline & & & & " & " & $6240-7000$ & 1.20 & 0.56 & 1.2 \\
\hline
\end{tabular}

addition of the photon counting errors and of the errors on the continuum level determination.

We used cross-correlation techniques as described in Márquez \& Moles (1996) to extract the gas kinematical information. The spatial section in the 2D spectrum with the highest $\mathrm{S} / \mathrm{N}$ ratio (excluding the nuclear region for Seyferts ${ }^{2}$ ) was used as a template for the cross-correlation. The errors refer to the determination of the velocity shift with respect to the template spectrum. They are shown as error bars in the velocity distributions. For the stellar velocity distributions, the spectra in the $\mathrm{CaT}$ region were used, with cross-correlation techniques (as described in Pérez et al. 2000, and references therein), using as templates for the cross-correlation the observed spectra of several stars. The errors refer to the determination of the velocity shift with respect to the template spectrum. For clarity, error bars in the stellar velocity distributions are not plotted, but they typically amount to $50 \mathrm{~km} \mathrm{~s}^{-1}$. The best $\mathrm{S} / \mathrm{N}$ data have been used to look for the presence of nuclear dips in the stellar velocity dispersions, and are presented in Márquez et al. (2003).

The sample of active galaxies is more thoroughly observed (more PAs, higher $\mathrm{S} / \mathrm{N}$ ). Nevertheless, we do not expect this to produce a severe bias concerning general properties, since only the data along the major axes are used to characterize the rotation curves and use them to derive the Tully-Fisher (TF) relation, and to estimate the metallicity ${ }^{3}$ of disk HII regions. With respect to the analysis of central structures, the same kind of $\mathrm{S}$-shaped deviations seems to be present along all major axes.

\footnotetext{
${ }^{2}$ Notice that in the nuclear regions of AGNs there are often several components, which we have not attempted to fit individually.

${ }^{3}$ As given by $[\mathrm{NII}] / \mathrm{H} \alpha$, see below.
}

Besides, for a number of galaxies (most of them active spirals), enough position angles have been observed and therefore a guess can be made on what specific feature (disk or bar) may be the origin of such deviations (see below).

\section{The shape of the rotation curves}

The gas velocity distributions measured along various slit position angles for all the galaxies of our sample are displayed in Figs. 2 to 51, with the exception of NGC 2811 for which the [NII] emission lines are not spatially extended, and were detected in the nuclear region only ( $\mathrm{H} \alpha$ is not detected in emission). The kinematical center and systemic velocities were determined as in Márquez et al. (2002): the kinematical center was defined as the cross section leading to symmetrical differences between the two horizontal branches of the rotation curve. That center corresponds, within our resolution, to the photometric center, i.e., the maximum in the continuum distribution along the slit, the biggest differences in position always being smaller than 2 pixels. The redshift corresponding to that kinematical center was adopted as the redshift of the system.

The stellar velocity distribution, when available, is shown superimposed on the gas data. Model rotation curves that better account for the observed data are shown in the figures as full lines; a normalized arctangent rotation curve function has been used, $v(R)=v_{0}+\frac{2}{\pi} v_{\mathrm{c}} \arctan (R)$, with $R=\left(r-r_{0}\right) / r_{\mathrm{t}}$, where $v_{0}$ is the velocity of the center of rotation, $r_{0}$ is the spatial center of the galaxy, $v_{\mathrm{c}}$ is the asymptotic velocity and $r_{\mathrm{t}}$ is a radius that corresponds to the transition region between the rising and the flat parts of the rotation curve (see Márquez et al. 2002). Their 
Table 4. Parameters derived from the velocity distributions along the major axes.

\begin{tabular}{|c|c|c|c|c|c|c|c|c|c|c|c|c|c|}
\hline Galaxy & (1) & (2) & $\mathrm{PA}_{\text {maj }}^{\mathrm{RC} 3}$ & $\begin{array}{r}\mathrm{PA}_{\text {maj }}^{\text {kine }} \\
(4)\end{array}$ & $\begin{array}{l}r_{G} \\
\left({ }^{\prime \prime}\right) \\
(5)\end{array}$ & $\begin{array}{c}v_{G} \\
\left(\mathrm{~km} \mathrm{~s}^{-1}\right) \\
(6)\end{array}$ & $\begin{array}{l}r_{1} \\
\left({ }^{\prime \prime}\right) \\
(7)\end{array}$ & $\begin{array}{c}v_{1} \\
\left(\mathrm{~km} \mathrm{~s}^{-1}\right) \\
(8)\end{array}$ & $\begin{array}{l}r_{\mathrm{m}} \\
\left({ }^{\prime \prime}\right) \\
(9)\end{array}$ & $\begin{array}{c}v_{\mathrm{m}} \\
\left(\mathrm{km} \mathrm{s}^{-1}\right) \\
(10)\end{array}$ & $\begin{array}{c}c z \\
\left(\mathrm{~km} \mathrm{~s}^{-1}\right) \\
(11)\end{array}$ & $\begin{array}{c}r_{0} \\
\left({ }^{\prime \prime}\right) \\
(12)\end{array}$ & $\begin{array}{c}v_{0} \\
\left(\mathrm{~km} \mathrm{~s}^{-1}\right) \\
(13)\end{array}$ \\
\hline UGC 1395 & 0.10 & 1.10 & - & 165 & 1.79 & 29 & 8.8 & 78 & 34 & 140 & 5139 & 4.5 & 132 \\
\hline IC 184 & 0.31 & 1.02 & 7 & 7 & 0.54 & 114 & 9.0 & 127 & 28 & 163 & 5332 & 0.5 & 120 \\
\hline IC 1816 & 0.07 & 1.16 & - & 150 & & & 3.0 & 177 & 21 & 159 & 5095 & 1.5 & 130 \\
\hline UGC 3223 & 0.25 & 1.15 & 80 & 68 & 0.80 & 77 & 1.6 & 111 & 21 & 250 & 4330 & 4.5 & 158 \\
\hline NGC 2639 & 0.22 & 1.26 & 140 & 135 & & & 6.0 & 229 & 33 & 310 & 3177 & 5.5 & 291 \\
\hline IC 2510 & 0.27 & 1.10 & 148 & 148 & & & 12.0 & 110 & 27 & 130 & 2529 & 7.5 & 119 \\
\hline NGC 3660 & 0.09 & 1.43 & 115 & 115 & 1.50 & 50 & 19.0 & 110 & 64 & 130 & 3441 & 4.5 & 155 \\
\hline NGC 4507 & 0.10 & 1.22 & - & 65 & 2.10 & 174 & 5.0 & 240 & 36 & 220 & 3689 & 1.5 & 130 \\
\hline NGC 4785 & 0.31 & 1.29 & 81 & 81 & 0.93 & 123 & 8.9 & 254 & 50 & 257 & 3672 & 1.5 & 197 \\
\hline NGC 5347 & 0.10 & 1.23 & 130 & $130 ?$ & - & - & - & - & - & - & 2382 & - & - \\
\hline NGC 5728 & 0.24 & 1.49 & 0 & 0 & 0.30 & 85 & 5.0 & 194 & 56 & 189 & 2813 & 1.5 & 180 \\
\hline ESO139-12 & 0.11 & 1.20 & 35 & 15 & & & 10.0 & 218 & 114 & 252 & 5139 & 5.5 & 180 \\
\hline NGC 6814 & 0.03 & 1.48 & - & - & - & - & - & - & - & - & 1556 & - & - \\
\hline NGC 6860 & 0.25 & 1.13 & 34 & 34 & 0.98 & 38 & 10.0 & 184 & 40 & 190 & 4437 & 3.0 & 157 \\
\hline NGC 6890 & 0.10 & 1.19 & 152 & 152 & 1.70 & 55 & 6.0 & 120 & 54 & 124 & 2528 & 2.5 & 148 \\
\hline NGC 6951 & 0.08 & 1.59 & 170 & 170 & & & 7.5 & 145 & 96 & 153 & 1414 & 2.0 & 130 \\
\hline NGC 151 & 0.34 & 1.57 & 75 & 75 & 0.90 & 78 & 7.5 & 280 & 100 & 227 & 3727 & 1.5 & 170 \\
\hline NGC 1357 & 0.16 & 1.45 & 85 & - & & & 5.5 & 160 & 55 & 137 & 2033 & 2.5 & 115 \\
\hline IC 454 & 0.28 & 1.24 & 140 & 140 & 0.40 & 48 & 16.0 & 227 & 44 & 228 & 3973 & 3.5 & 155 \\
\hline NGC $2712^{* *}$ & 0.26 & 1.46 & 178 & - & 2.5 & 106 & 20 & 150 & 49 & 152 & 1833 & 1.5 & 115 \\
\hline NGC 2811 & 0.46 & 1.40 & 20 & - & - & - & - & - & - & - & 2514 & - & - \\
\hline NGC 3571 & 0.46 & 1.48 & 94 & - & 0.90 & 85 & 2.0 & 143 & 26 & 153 & 3771 & 0.8 & 106 \\
\hline NGC $3835^{* *}$ & 0.39 & 1.29 & 60 & - & 0.90 & 32 & 25 & 190 & 48 & 198 & 2452 & 6.5 & 150 \\
\hline NGC $4162^{* *}$ & 0.22 & 1.37 & 174 & - & 1.3 & 61 & 22 & 159 & 48 & 160 & 2542 & 2.5 & 130 \\
\hline NGC $4290^{* *}$ & 0.16 & 1.37 & 90 & - & 0.80 & 35 & 11 & 180 & 31 & 180 & 3035 & 3.5 & 145 \\
\hline NGC 6012 & 0.14 & 1.32 & 150 & 168 & - & - & 13.6 & 99 & 64 & 111 & 1988 & 8.2 & 150 \\
\hline NGC $6155^{* *}$ & 0.16 & 1.13 & 145 & - & 1.5 & 30 & 12 & 80 & 41 & 90 & 2429 & 5.5 & 95 \\
\hline NGC 7328 & 0.44 & 1.31 & 88 & 88 & 2.0 & 73 & 8.0 & 150 & 77 & 150 & 2793 & 2.0 & 107 \\
\hline
\end{tabular}

(1) and (2) inclination and isophotal diameter, respectively, as given in RC3 (in its units); (3) major axis position angle as given in RC3; (4) kinematical major axis as determined from our data; (5) distance to the center of the point out to which the solid body rotation is seen; (6) the velocity measured at $r_{G} ;(7)$ distance to the center of the point where the first maximum peak in velocity; (8) the velocity measured at $r_{1}$; (9) maximum distance to the center in the velocity distribution; (10) the velocity measured at $r_{\mathrm{m}} ;(11)$ systemic redshift as measured from our data; (12) and (13) parameters for the model rotation curve (see text).

* Taken from NED.

** Excepting the last two columns, the values are from Márquez et al. (1996).

corresponding parameters are given in Table 4. These do not correspond to best fits, but only to the models which by eye fit best the data points along the various PA, with priority given to the major axis. This model has been used for the sake of comparison with Márquez et al. (2002, hereafter M111) and is quite appropriate to describe the general shape of the rotation curves of spiral galaxies, in particular concerning the parameters used for Tully-Fisher studies (Courteau 1997; M111 and references therein). These models are also used to constrain the kinematical major axes, for which the use of a more refined mathematical form is not critical. In two cases (UGC 3223 and ESO 139-12) the kinematical major axis is found to be 


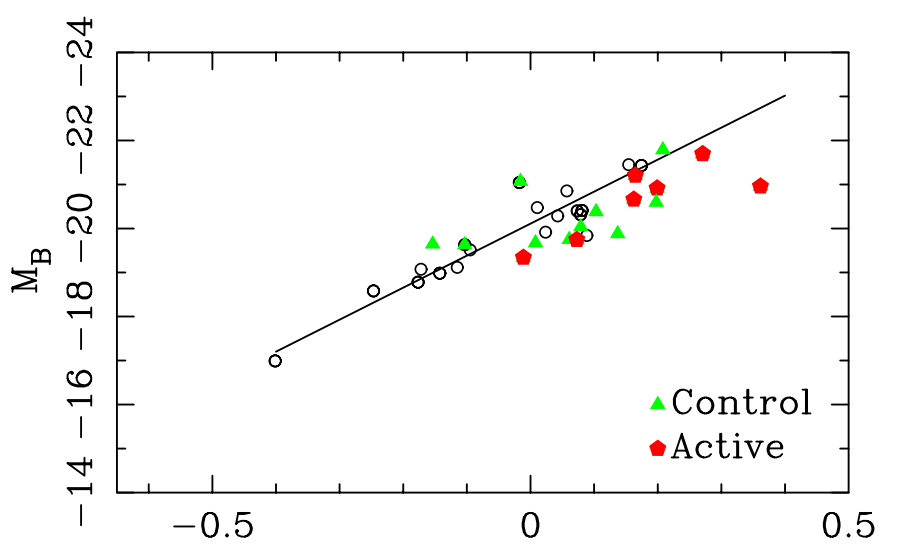

$$
\log \left(W_{\max }\right)-2.5
$$

Fig. 1. Tully-Fisher relation for the galaxies of our sample (pentagons for active galaxies and triangles for non-active ones), together with the isolated galaxies of the M111 sample (empty circles). $M_{B}$ is the $B$ corrected absolute magnitude, and $W_{\max }=2 \times V_{\max }\left(\mathrm{km} \mathrm{s}^{-1}\right)$ is the total amplitude of the rotation curve. The line indicates the TF relation by Tully \& Pierce (2000).

different from the photometric major axis (taken from the RC3 catalogue). For UGC 1395, IC 1186 and NGC 4507, no photometric PA is given in the RC3 (see Table 4 for the major axis PA derived from our kinematical data). In a number of cases the number of slit positions and data points were not sufficient to obtain a full kinematical model (see below).

To check whether the galaxies of the DEGAS sample obey the Tully-Fisher (TF) relation, we plot in Fig. 1 the absolute magnitude $B_{T 0}$ (obtained from the corrected $B$ magnitude given in the RC3) as a function of the rotation amplitude. The latter was estimated at $R_{\mathrm{opt}} \approx 1.2 \times R_{25}$. The extrapolation of the model curve was used to get $V_{\mathrm{opt}}=V\left(R_{\mathrm{opt}}\right)$, as in M111. It is clear from the figure that active and control galaxies populate the same area of the TF plane, indicating that their overall kinematical properties are similar. The small offset along the $x$-axis indicates that, within $1 \sigma$, Seyfert galaxies have median rotation velocities higher that control galaxies, for the same median luminosity. We also see that they are defining, within $1 \sigma$, the same TF relation as the isolated galaxies discussed in M111 (the DEGAS sample comprises earlier spirals, that tend to be more luminous and therefore are expected to be placed in the upper region of the diagram). We notice however that, even within $1 \sigma$, most of the active and control galaxies discussed here are located below the TF line defined in M111. The reason could be the differences in morphological content between the samples in M111 and here, or the need for more accurate absolute magnitudes than those provided by the RC3. Indeed, it also could be just an artifact of the small number statistics.

We give below details on the velocity curves of each galaxy with indications of particular aspects such as departures from the general rotation curve or differences between the gas and star velocity distributions. Most galaxies of our sample show rotation curves in global agreement with circular motions, but including some interesting details. S-shape structures are quite common near the nuclear regions, sometimes with different amplitudes on either side, and often showing a velocity dip along the minor axis. To better see the behaviour of such departures, the model rotation curve has been subtracted from the data for each PA and the corresponding residuals have been inspected. In fact, zero velocity residuals are expected along the major axis when the inner disk axis has the same major axis than the large scale one; a rotation-like sinusoidal feature is expected when these two axes are misaligned, with amplitudes differing for the different PAs. This allows hence a better discrimination between nuclear bar or nuclear disk as the origin of such S-shape features, although for a definitive conclusion integral field spectroscopy is required. The departure from simple rotation explained for some galaxies by the presence of a bar and/or of a spiral arm are usually also based on our previously published optical (Márquez \& Moles 1996) or infrared (Márquez et al. 1999) images.

\subsection{Active galaxies}

UGC 1395 (Fig. 2) has a rather smooth rotation curve. Only at $\mathrm{PA}=45^{\circ}$ and $135^{\circ}$ the observed velocity distribution slightly departs from the overall rotation pattern. There is no entry for the photometric major axis in the RC3. Our data indicate that the kinematical major axis is at $\mathrm{PA} \simeq 165^{\circ}$. The gas and stellar rotation curves look pretty similar along $\mathrm{PA}=80^{\circ}$ and $125^{\circ}$; but along PA $=35^{\circ}$ the stars follow very well the model \pm 3 arcsec from the nucleus, whereas the gas velocity distribution deviates from this curve by about $30 \mathrm{~km} \mathrm{~s}^{-1} 2$ arcsec NE of the nucleus (see also Fig. 3). This occurs at the position of the bent dust lane structure originating at the NE extremity of the nuclear bar (Pogge \& Martini 2002). The residual velocity plots ${ }^{4}$ indicate that the S-shape structure interior to \pm 5 arcsec, more clearly visible along $\mathrm{PA}=125^{\circ}$ and $\mathrm{PA}=35^{\circ}$, may originate in the inner bar structure (see Fig. 3).

The kinematical and photometric major axes in IC 184 are coincident. The gas (Fig. 4) shows a strong asymmetric departure from the model rotation curve, specially along $\mathrm{PA}=7^{\circ}$ and $97^{\circ}$ (major and minor axis, respectively) out to $\pm 10 \operatorname{arcsec}$ from the center, while the stellar velocities follow quite well the model along $\mathrm{PA}=97^{\circ}$ (the stellar velocity distribution along $\mathrm{PA}=7^{\circ}$ is given in Márquez et al. 2003). This is illustrated in the enlarged figure of the central zone along $\mathrm{PA}=97^{\circ}$ and in Fig. 1 of Márquez et al. (2003). For the gas, the $\approx 100 \mathrm{~km} \mathrm{~s}^{-1}$ difference between the data points and the rotation model $\mathrm{N}$ and $\mathrm{NE}$ of the nucleus along $\mathrm{PA}=7^{\circ}$ and $37^{\circ}$ are most probably due to the bar and north spiral arm; the bump observed along $\mathrm{PA}=97^{\circ}$ is most probably also due to the bar. Note that a drop in the stellar velocity dispersion is observed in this galaxy (Márquez et al. 2003). The residuals of the gas velocity distributions along $\mathrm{PA}=37^{\circ}$ and $\mathrm{PA}=157^{\circ}$ in the inner \pm 1 arcsec (see Fig. 5) hint for the presence of a nuclear disk, as those found in some active galaxies (see Márquez et al. 2003 and references therein). Although IC 184 has been selected as an isolated spiral following the criteria defined for the DEGAS sample, we recently came upon a paper indicating that it has several

\footnotetext{
${ }^{4}$ Available in electronic form only.
} 

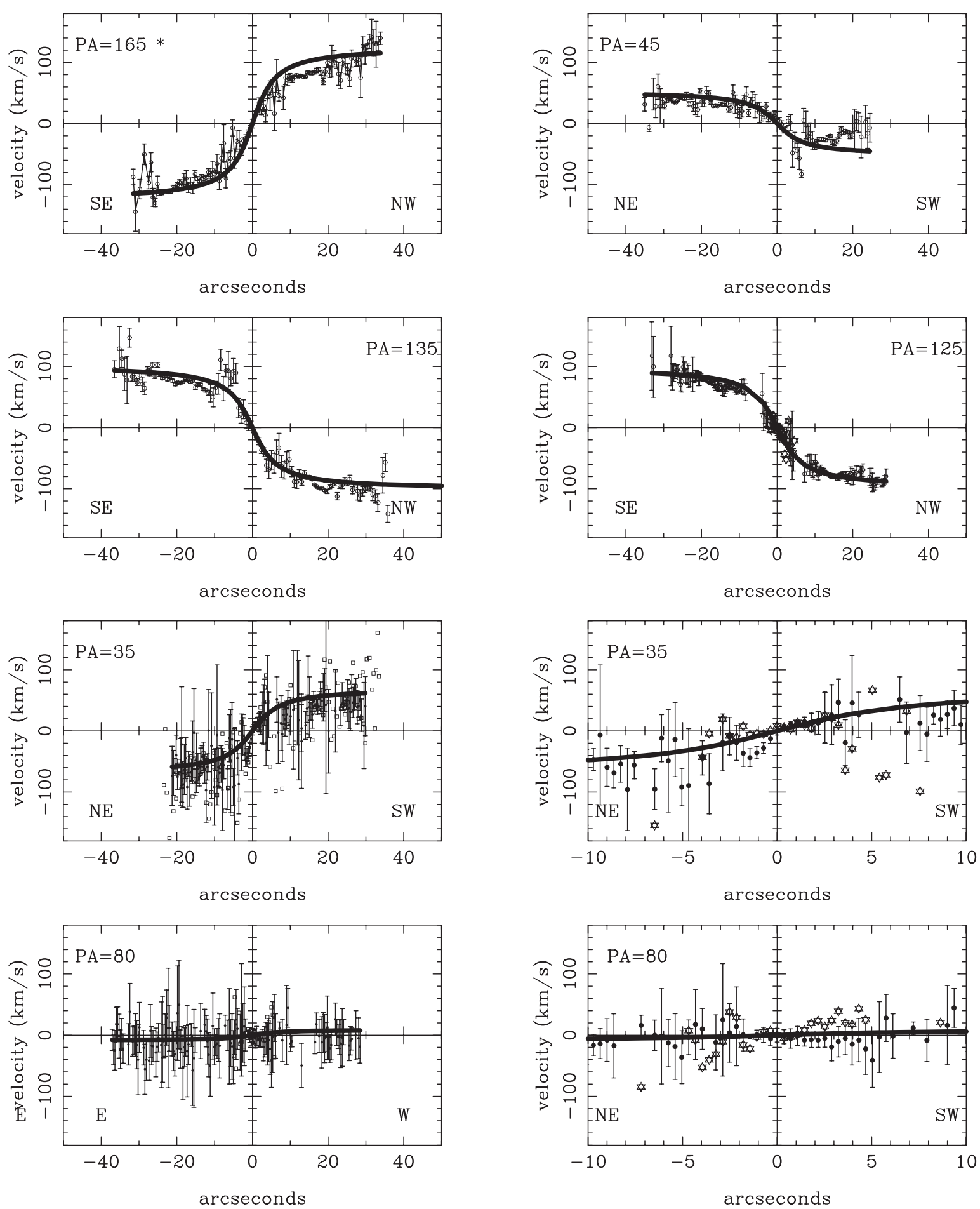

Fig. 2. Velocity distributions for UGC 1395. As for all similar figures, gas velocities derived from $\mathrm{H} \alpha$ and $\mathrm{H} \beta$ are shown as black dots (with error bars) and empty light circles respectively. Stellar velocities are shown with stars. For PA $=35$ and $80^{\circ}$ an enlarged part of the central region is plotted to see better the corresponding stellar kinematics. An asterisk after the PA indicates the slit position angle coinciding with the major axis. The line corresponds to the rotation model which best reproduces the data.

spectroscopically confirmed faint companions (Kollatschny \& Fricke 1987).

The ionized gas in IC 1816 (Fig. 6) presents a rather smooth velocity distribution along the two perpendicular PAs. Our data indicate that the kinematical major axis is at $\mathrm{PA} \simeq 150^{\circ}$. No value is given in the RC3 for the photometric major axis. The measured velocities north of the nucleus along $\mathrm{PA}=0^{\circ}$ are about $50 \mathrm{~km} \mathrm{~s}^{-1}$ higher than expected, probably due to the 

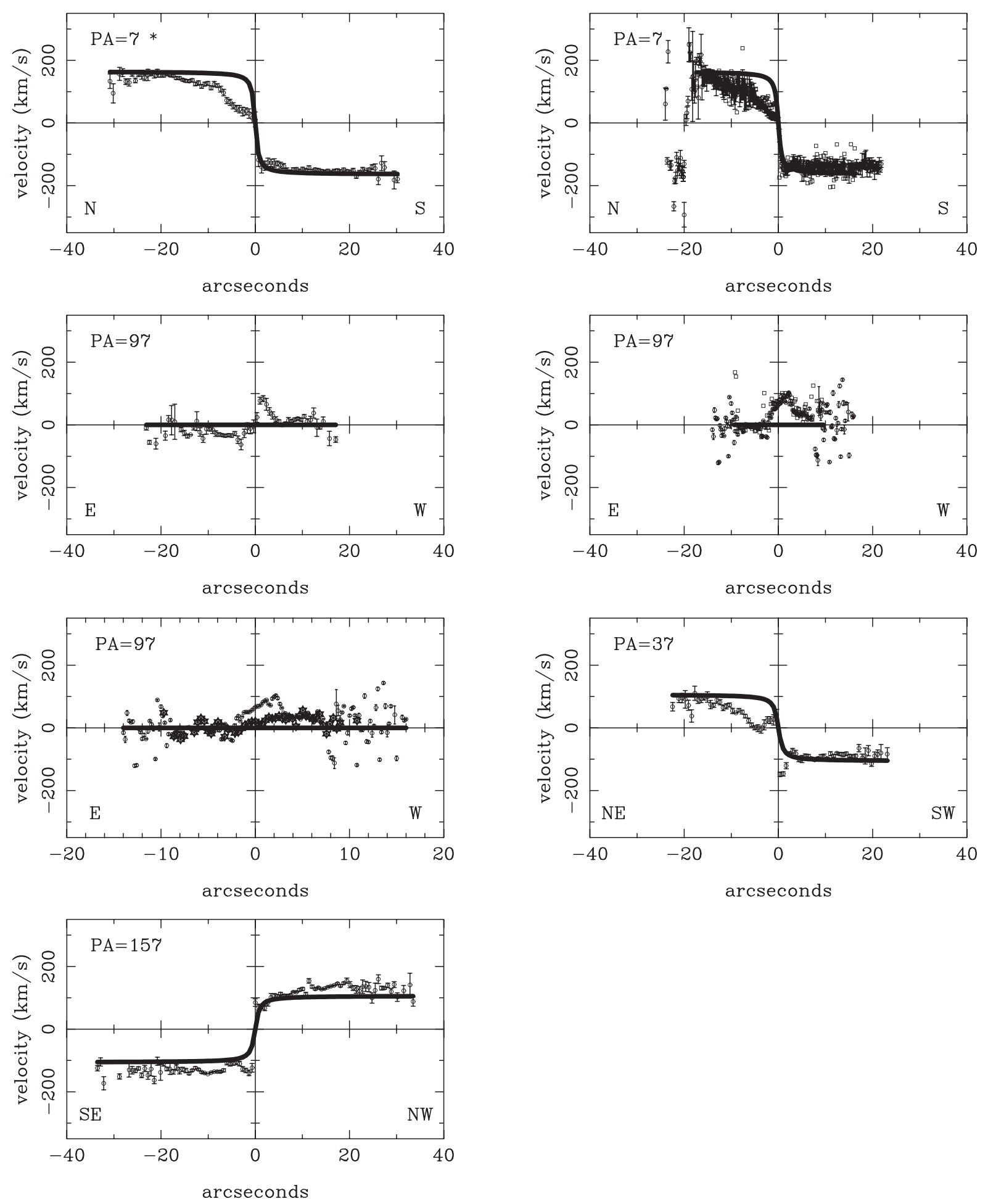

Fig. 4. Velocity distributions for IC 184. The two sets of two curves along PA = 7 and PA $=97$ correspond to the LC (left) and WHT (right) data. An enlarged part of the central zone is shown with stars indicating the stellar component.

presence of a spiral arm. The small counter-rotation observed roughly \pm 3 arcsec from the nucleus could be due to the presence of the inner bar, but it could also trace the presence of a nuclear disk (see Fig. 7).
UGC 3223 (Fig. 8) presents a rather smooth gas velocity distribution with a major axis in better agreement with $\mathrm{PA} \simeq 70^{\circ}$ than with the photometric major axis given in the RC3 $\left(\mathrm{PA}=80^{\circ}\right)$. An $\mathrm{S}$-shaped structure is also visible in this galaxy, 
IC 1816

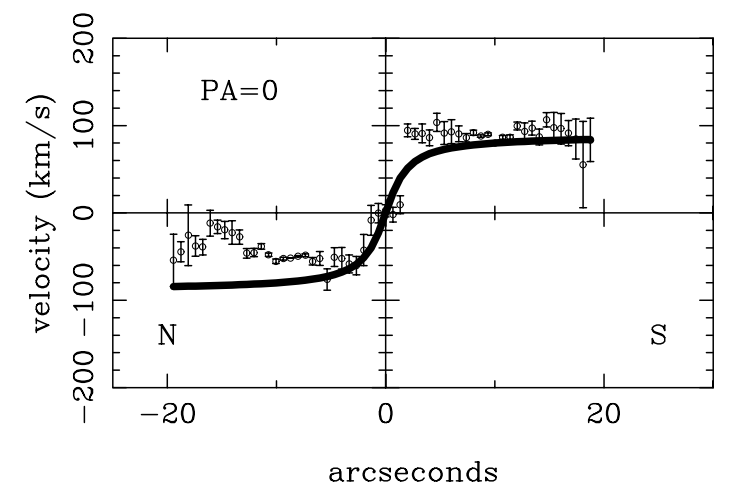

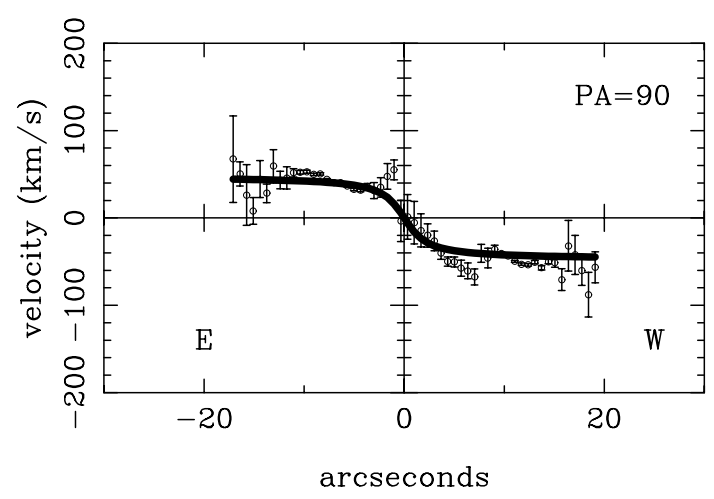

Fig. 6. Velocity distributions for IC 1816.

specially along $\mathrm{PA}=20^{\circ}$ and also along $\mathrm{PA}=170^{\circ}$ (close to the kinematical minor axis) where it is asymmetric in amplitude. The stellar and gas velocity curves in the central region along PA $=80^{\circ}$ are given in Fig. 2 of Márquez et al. (2003), where the presence of a drop in the stellar velocity dispersion is reported. An S-shape structure at \pm 4 arcsec in the residuals, observed in all position angles but more clearly visible at PA = $20^{\circ}$, can be attributed to the inner bar (see Fig. 9).

NGC 2639 (Fig. 10) shows smooth gas and star rotations along the three PA for which we have data. Along PA $=135^{\circ}$ (the photometric major axis is at $\mathrm{PA}=140^{\circ}$ ), the stars follow the model throughout while the gas shows some wiggles (see Fig. 11). Along $\mathrm{PA}=0^{\circ}$, the gas and stellar velocities follow each other, with an apparent counter-rotation in the innermost \pm 1 arcsec, which is also visible for the gas along $\mathrm{PA}=45^{\circ}$. The counter-rotation is more clearly evidenced in the residuals, which show that it is also present along $\mathrm{PA}=135^{\circ}$ (see Fig. 11). The rotation curves are given for the gas and stars along PA = $45^{\circ}$ in Márquez et al. (2003), where the presence both of a nuclear disk from HST images and of a drop in the stellar velocity dispersion are discussed. The residuals in the inner $\pm 2 \operatorname{arcsec}$ seem to be due to the inner bar.

IC 2510 (Fig. 12) shows rather smooth gas rotation, with small deviations about 5-12 arcsec SE of the nucleus along $\mathrm{PA}=148^{\circ}$ and $8-15 \operatorname{arcsec} \mathrm{NW}$ of the nucleus along $\mathrm{PA}=118^{\circ}$, both probably due to the presence of a bar. A small asymmetric S-shape structure is present in the central \pm 3 arcsec, more clearly detected at position angles other than the major axis in Fig. 12, and along all the PA in the residuals (see Fig. 13). This structure coincides with a region immediately SW of the major axis, which is an incomplete arc-like structure visible in the HST image. The global shape of the rotation curve along the major axis agrees with that given by Persic \& Salucci (1995).

NGC 3660 (Fig. 14), for which the kinematical and photometric major axes coincide, shows a stellar rotation pattern that is well fit by the rotation model along $\mathrm{PA}=115^{\circ}$. The velocity distribution of the gas shows a bump of about $100 \mathrm{~km} \mathrm{~s}^{-1}$ in the region between 3 and $5^{\prime \prime}$ to the NW of the center, with residuals around zero for $\mathrm{PA}=145^{\circ}$ (see Fig. 15); this could indicate the presence of a nuclear disk perpendicular to this PA. A possible stellar counter-rotation is seen in the inner \pm 1 arcsec along
$\mathrm{PA}=85^{\circ}$, but the stellar data for this PA are very noisy and no firm conclusion can be extracted. The outer parts of the rotation curve of the gas are not well accounted for by the model, probably due to the crossing of various spiral arms.

For NGC 4507 (Fig. 16) we estimate a kinematical major axis at $\mathrm{PA} \sim 65^{\circ}$ (no value is given in the RC3 for the photometric axis). The gas velocity distribution along $\mathrm{PA}=51^{\circ}$ is in general well represented by the model, but it is somewhat wiggly along $\mathrm{PA}=141^{\circ}$, where it shows an asymmetric Sshape structure with a strong dip (about $100 \mathrm{~km} \mathrm{~s}^{-1}$ ) between 2 and $10 \operatorname{arcsec} \mathrm{SE}$ of the nucleus (see Fig. 17).

NGC 4785 (Fig. 18) shows a smooth rotation pattern for the gas along $\mathrm{PA}=81^{\circ}$ (the major axis), with a velocity drop about 8 arcsec $\mathrm{W}$ of the nucleus, probably due to the bar and/or beginning of the spiral arm; the velocity field has a number of wiggles along $\mathrm{PA}=171^{\circ}$, with an S-shape structure in the inner \pm 10 arcsec, deeper to the South (see Fig. 19); no obvious morphological feature is seen in this region in our infrared image.

NGC 5347 (Fig. 20) has too sparse data to be analyzed in detail. Notice however that an apparent counter rotation is noticed in the central \pm 5 arcsec along both the photometric major $\left(\mathrm{PA}=130^{\circ}\right)$ and minor $\left(\mathrm{PA}=40^{\circ}\right)$ axes (see the inset shown in Fig. 21). This region coincides with the dust lane seen in the HST map (Pogge \& Martini 2002).

For NGC 5728 (Fig. 22), the photometric and kinematical major axes are in excellent agreement $\left(\mathrm{PA}=0^{\circ}\right)$. The gas kinematics show strong departures from the expected, standard disk rotation model along the four observed PA. The velocity gradient is very steep in the few arcseconds around the nucleus. Our data are in agreement with the previous determination by Schommer et al. (1988), who derived a position angle for the major axis at $2 \pm 5^{\circ}$. Our better spatial resolution allows us to detect a strong velocity discontinuity a few arcseconds on either side of the nucleus which is seen along $\mathrm{PA}=90^{\circ}$ (corresponding to the minor axis) and $\mathrm{PA}=150^{\circ}$ in Fig. 22 and along all the PAs in the residuals (see Fig. 23). These position angles are close to those used by Emsellem et al. (2001) to derive stellar velocity distributions within the central \pm 5 arcsec; the resulting stellar kinematics are very smooth, and show no velocity discontinuity. This seems to show evidence for a massive central gaseous disk, decoupled from the stars, somewhat similar to the one we found in NGC 6951 (Pérez et al. 2000). 

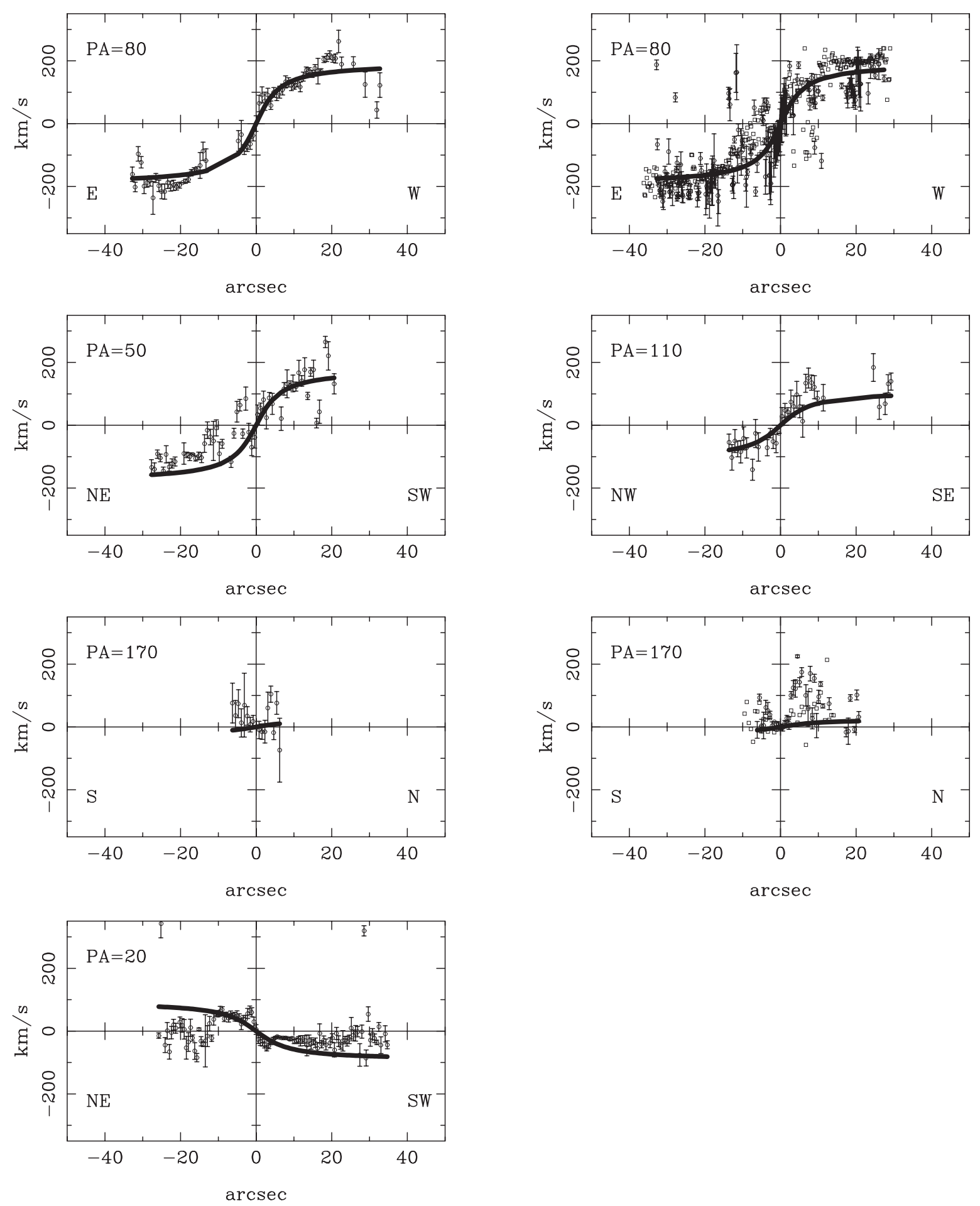

Fig. 8. Velocity distributions for UGC 3223. The two sets of curves along PA $=80^{\circ}$ and $\mathrm{PA}=170^{\circ}$ correspond to $4 \mathrm{~m}$ class telescopes (right) and ESO (left) data.

However, the presence of two components within the inner \pm 10 arcsec detected with a higher spectral resolution by Prada \& Gutiérrez (1999) could have an influence on the shapes of the rotation curves.
ESO 139-12 (Fig. 24) appears to be the only unbarred galaxy in our subsample of active galaxies, even after analysis of the infrared images (Márquez et al. 1999, 2000) and shows smooth rotation along all observed PA. The differences 

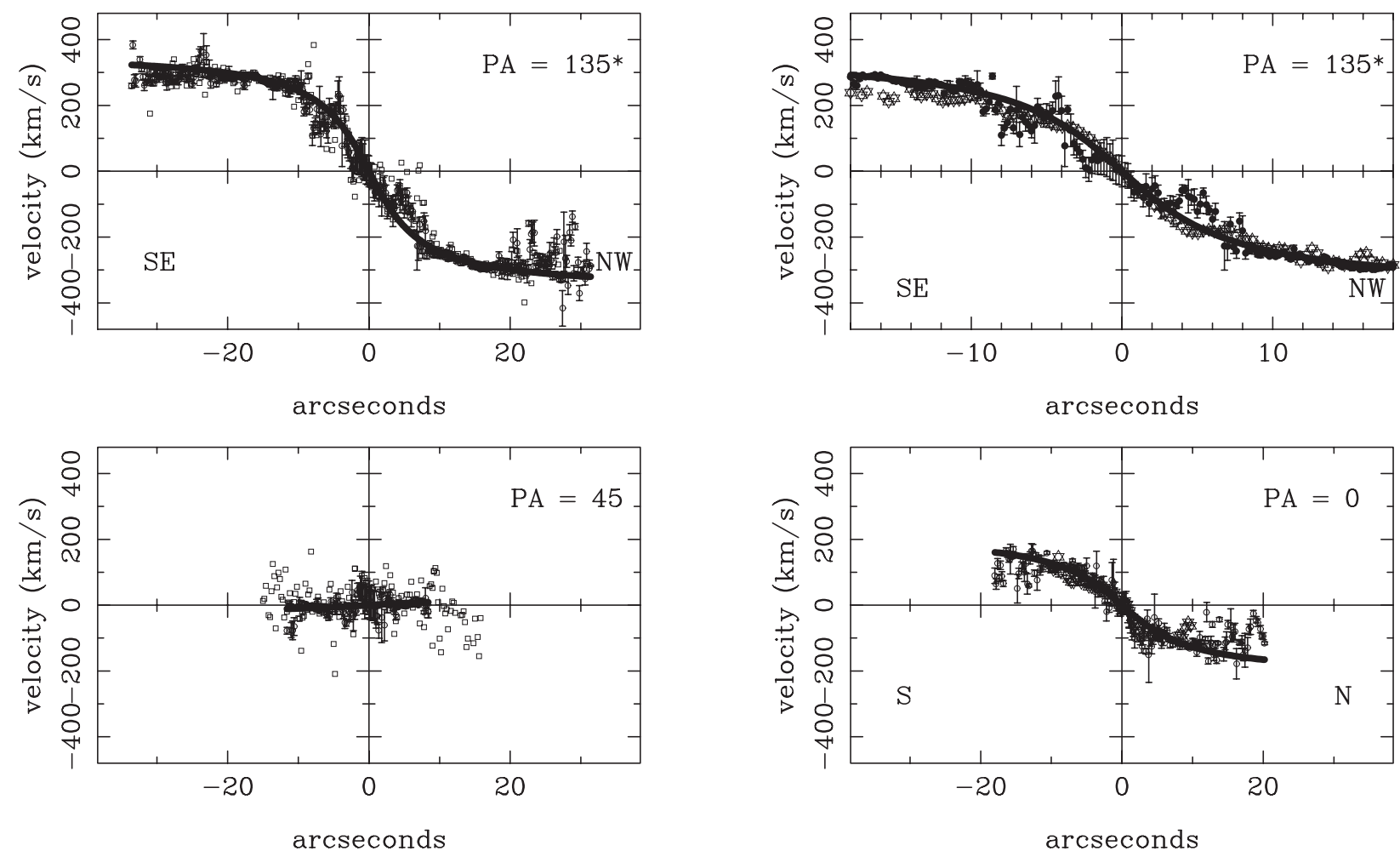

Fig. 10. Velocity distributions for NGC 2639. The top right figure shows an enlargement of the central region with the stellar velocities indicated in blue.

IC 2510
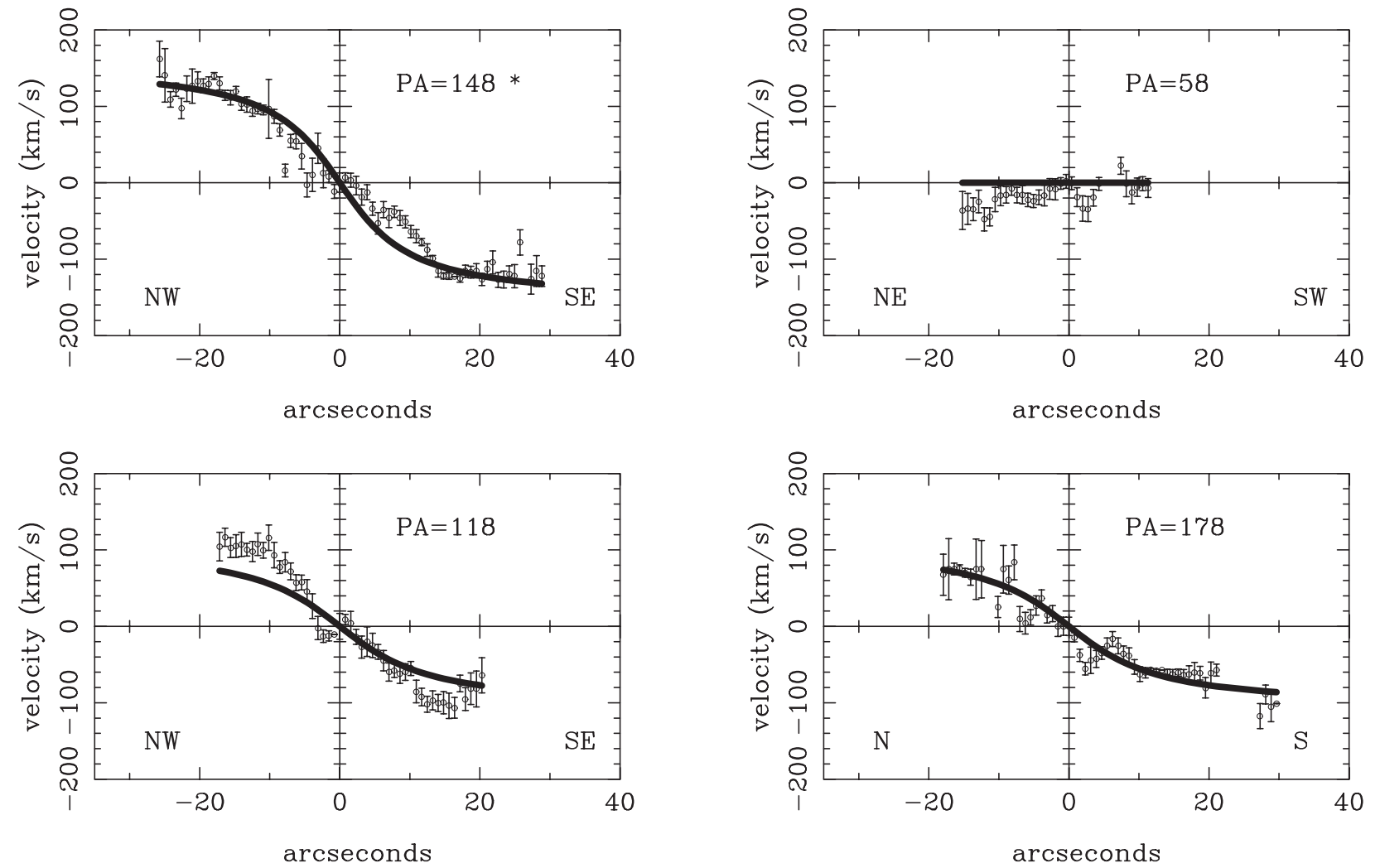

Fig. 12. Velocity distributions for IC 2510 . 

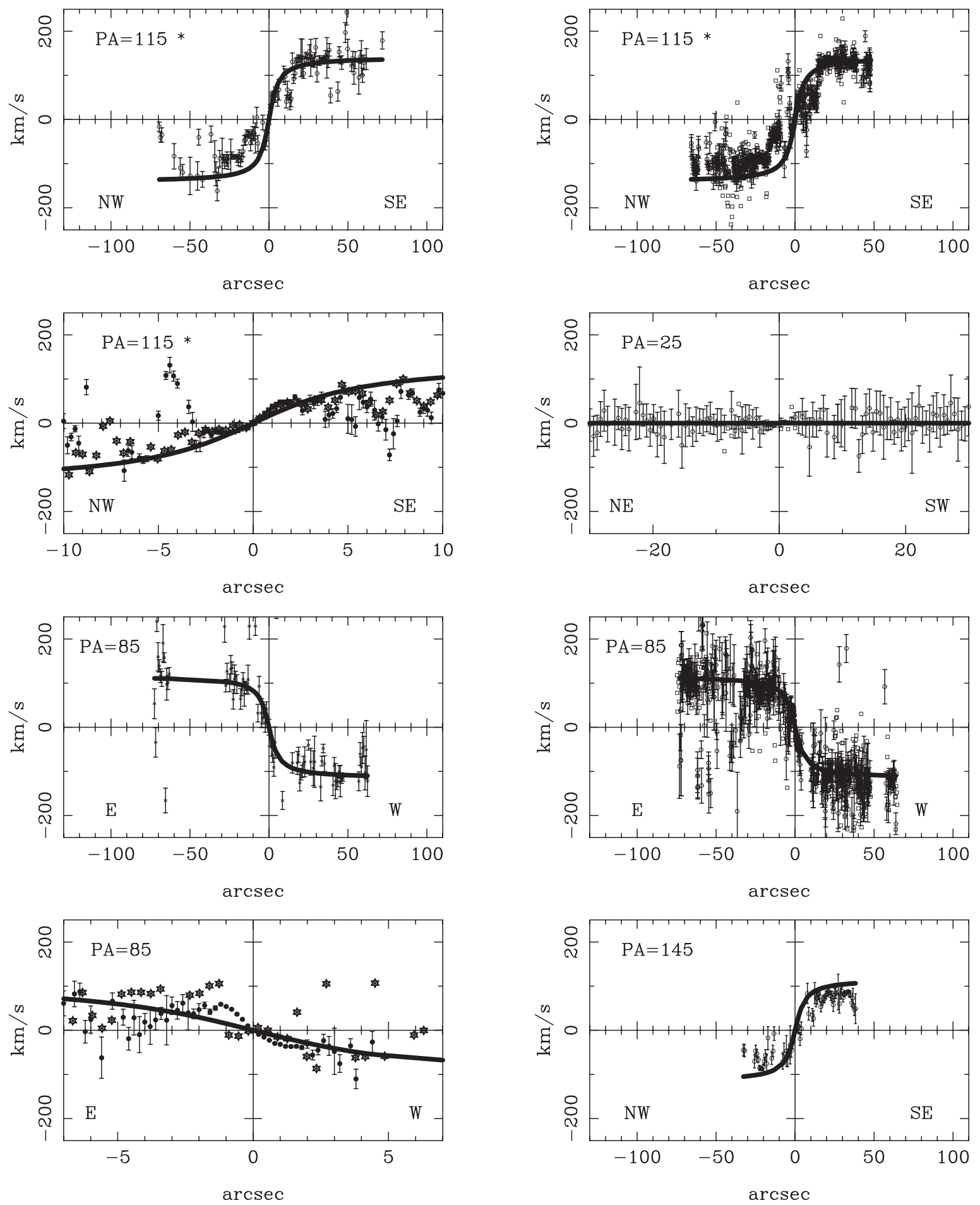

Fig. 14. Velocity distributions for NGC 3660. The ESO96 (left) and WHT99 (right) curves are on the top line for PA = 115. A zoom of the central region of the WHT99 data along PA $=115$ is shown in the second line, together with the curve along PA $=25$ where a few velocities from the $\mathrm{H} \beta$ line are visible in the very center. The ESO96 (left) and WHT99 (right) curves are on the third line for PA = 85. A zoom of the central region of the WHT00 data along PA $=85$ is shown in the fourth line.

between the model and the observed gas kinematics are minimized when the kinematical major axis is placed at $\mathrm{PA}=15^{\circ}$, instead of $35^{\circ}$ as given in the RC3 for the photometric axis. An S-shape structure is detected in the inner few arcseconds, specially visible along $\mathrm{PA}=120^{\circ}$ and $125^{\circ}$. This could correspond to an incomplete spiral-like feature detected in the innermost 1." 8 in the HST image. The residuals indicate that this 
NGC 4507

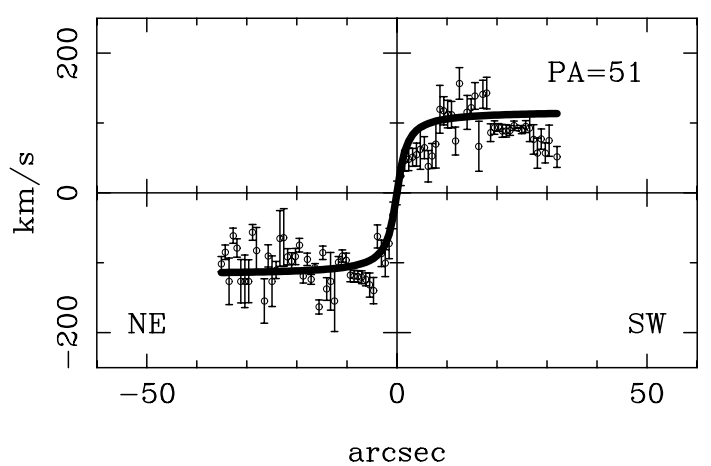

Fig. 16. Velocity distributions for NGC 4507.

NGC 4785

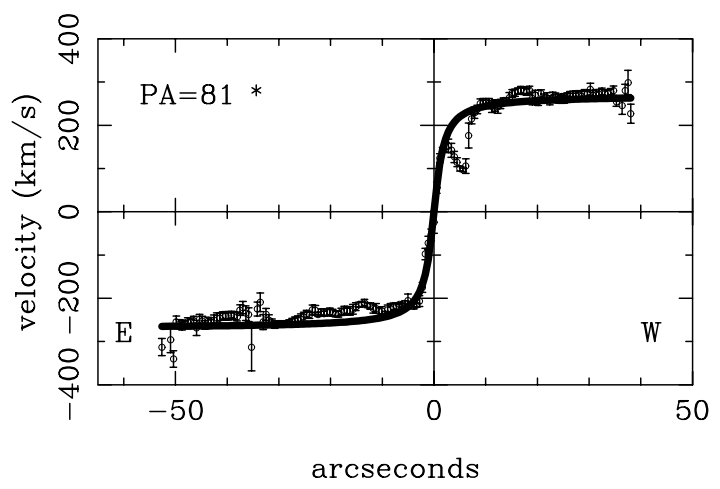

Fig. 18. Velocity distributions for NGC 4785.

NGC 5347

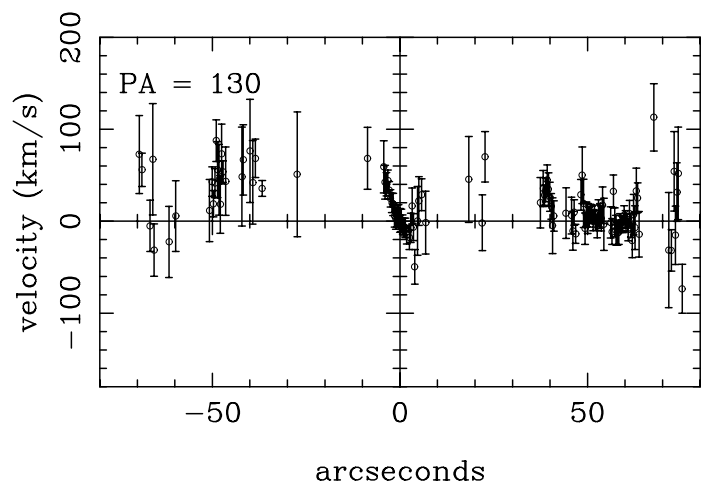

Fig. 20. Velocity distributions for NGC 5347.

feature may be produced by a nuclear disk with a minor axis close to $\mathrm{PA}=60-65^{\circ}$ (see Fig. 25).

NGC 6814 (Fig. 26) is seen too close to face-on for any kinematical model to be applied. Note however that an asymmetric S-shaped structure in the gas velocities is observed in the central arcseconds of all the spectra, with a dominant northern component reaching maximum amplitude about 4 arcsec from the nucleus. This feature may be due to the presence of some structure within the bar, most probably also related to the drop detected in the stellar velocity dispersion (Márquez et al. 2003). Notice that the stars follow the gas along PA $=30^{\circ} \mathrm{SW}$ of the nucleus but not in the other direction. Stellar and gas rotation
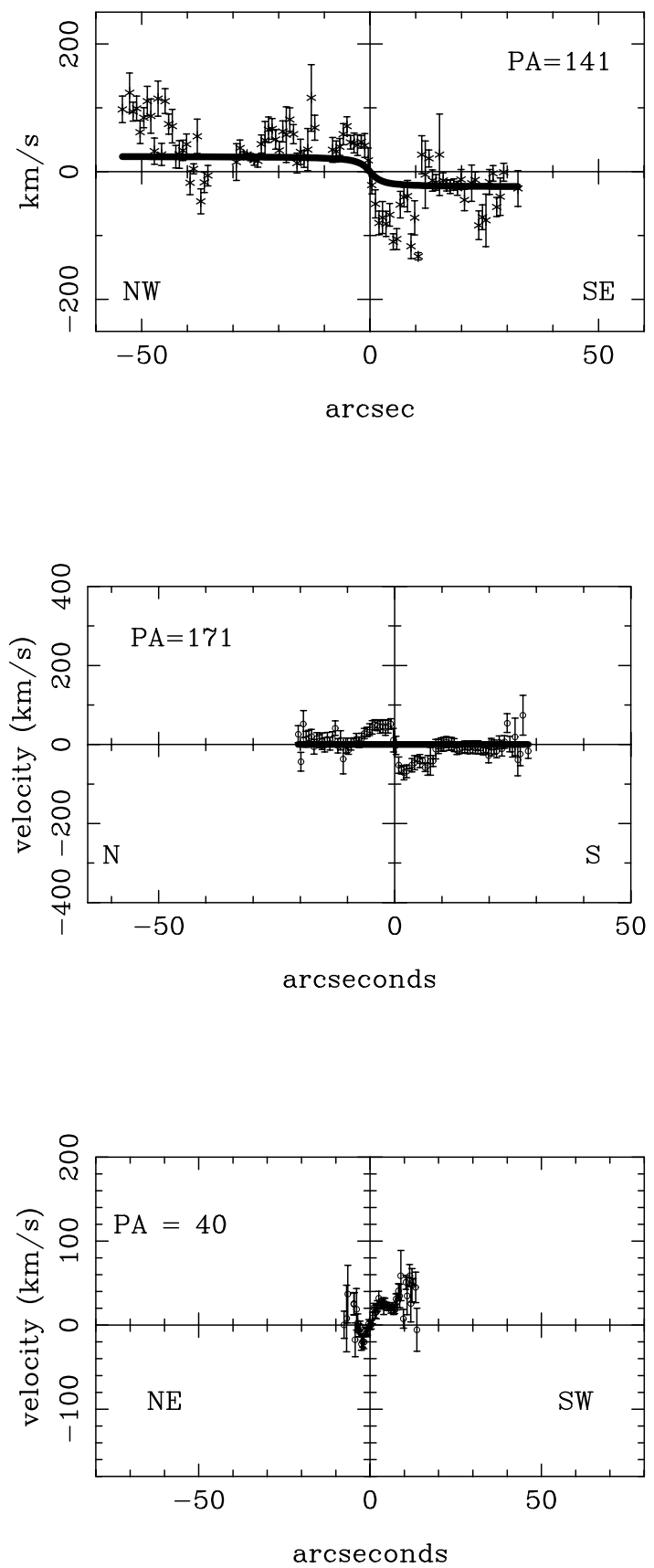

curves along $\mathrm{PA}=120^{\circ}$ are also presented in Márquez et al. (2003), where the presence of a broad drop in the stellar velocity dispersion is discussed.

NGC 6860 (Fig. 27) has kinematical and photometric major axes in excellent agreement and shows smooth gas rotation along its major axis but a number of features, most probably due to the presence of the bar, are observed along the other PAs, such as an asymmetric S-like feature close to the nucleus, with departures from circular rotation up to $100 \mathrm{~km} \mathrm{~s}^{-1}$ along the minor axis $\left(\mathrm{PA}=124^{\circ}\right)$. The velocity gradient in the central region along $\mathrm{PA}=64^{\circ}$ (which crosses the bar) is steeper than expected from a simple rotation model. The residuals along the 

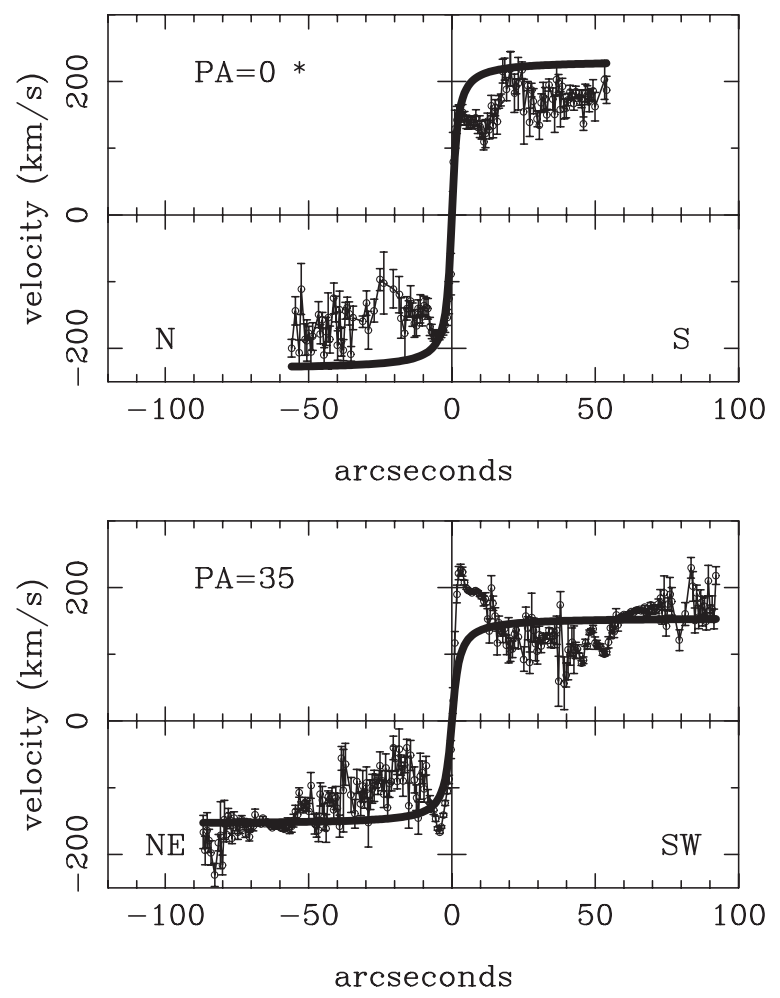

Fig. 22. Velocity distributions for NGC 5728.

observed PAs hint for the structure in these inner \pm 4 arcsec to be due to the inner bar (see Fig. 28).

NGC 6890 (Fig. 29) shows quite a regular circular gas rotation except along its minor axis $\left(\mathrm{PA}=62^{\circ}\right)$ where a wide velocity dip is observed $\mathrm{NE}$ of the nucleus, corresponding to one of the sides of an asymmetric S-like feature which is present along the other PAs (this is more clearly seen in the residuals, Fig. 30). The photometric and kinematical major axes are the same for this galaxy.

\subsection{Non-active galaxies: New data}

NGC 151 (Fig. 31) shows overall regular circular rotation along its major axis at $\mathrm{PA}=75^{\circ}$ (coincident with the photometric axis) but there are velocity dips along $\mathrm{PA}=151^{\circ}$ and $165^{\circ}$ just NW of the nucleus. These dips correspond to an asymmetric $\mathrm{S}$-shaped structure, with a maximum amplitude of almost $\pm 200 \mathrm{~km} \mathrm{~s}^{-1}$ along PA $=151^{\circ}$, which is seen in the residuals for all the PAs (see Fig. 32). Such kinematical behavior could be created by a secondary bar $2^{\prime \prime} .5$ long at $\mathrm{PA}=68^{\circ}$ visible in the HST image (see Fig. 34 in Márquez et al. 1999).

NGC 1357, an optically unbarred galaxy as classified in the RC3, shows an overall smooth gas rotation along PA = $85^{\circ}$, corresponding to the photometric major axis (Fig. 33). A S-like feature is visible mostly in the left side of the curve (see Fig. 34).

IC 454, for which we determine a kinematical major axis coincident with the photometric one ( $\mathrm{PA}=140^{\circ}$ as in the $\left.\mathrm{RC} 3\right)$, shows somewhat wiggly gas rotation curves along all the four
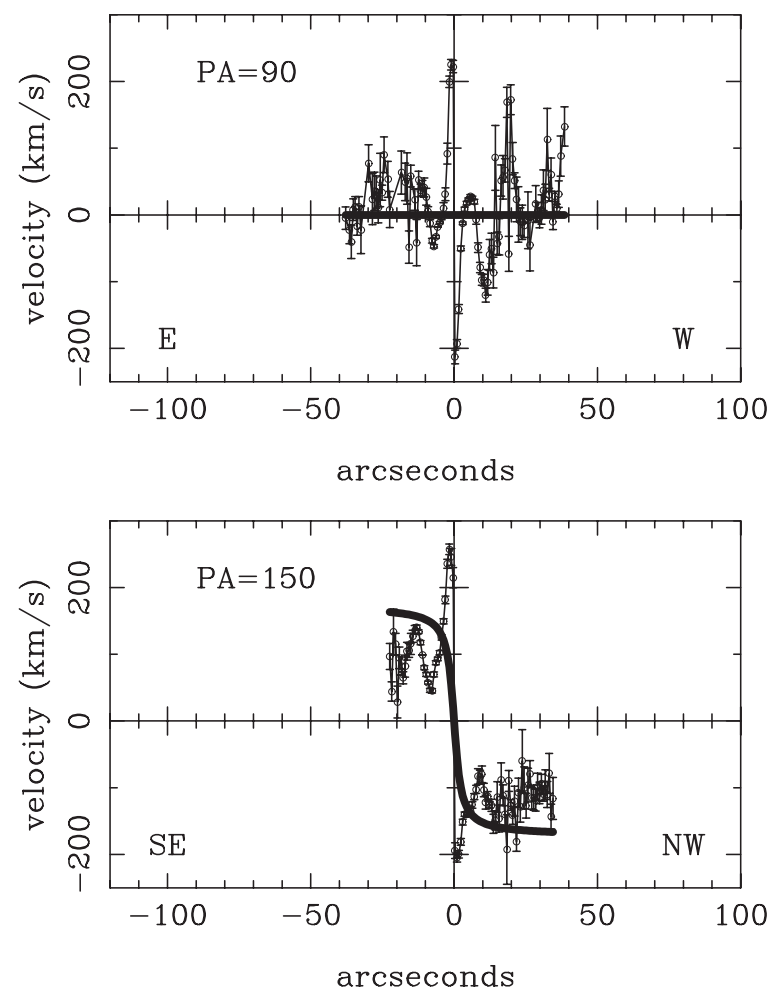

observed PA (Fig. 35). An asymmetric S-like feature is also present in this galaxy, most clearly visible along $\mathrm{PA}=110^{\circ}$ in Fig. 35 but present in the residuals for all PAs (see Fig. 36). It produces a dip of about $100 \mathrm{~km} \mathrm{~s}^{-1}$ in the velocity distribution, $\mathrm{SW}$ of the nucleus along the minor axis $\left(\mathrm{PA}=50^{\circ}\right)$. The residuals seem to indicate that this feature is due to the nuclear bar.

NGC 2811 shows weak $\mathrm{H} \alpha$ in absorption and [NII] in emission in the very central region, and no emission lines further out, so it is not possible to analyze its kinematics.

NGC 3571 (Fig. 37) shows weak extended emission, only detectable along $\mathrm{PA}=94^{\circ}$ (its photometrical major axis) with an overall regular gas rotation. Departures from the model are too noisy to extract any conclusion (Fig. 38).

For NGC 6012 the best model (Fig. 39) requires the kinematical major axis to be at $\mathrm{PA}=150^{\circ}$, as determined from near-infrared data (Márquez et al. 1999), whereas a value of $\mathrm{PA}=168^{\circ}$ is given in the RC3. The gas kinematics are compatible with overall circular rotation along $\mathrm{PA}=168^{\circ}$ except for a velocity drop about $30 \operatorname{arcsec} \mathrm{S}$ of the nucleus. However, the velocity distribution is less regular along $\mathrm{PA}=78^{\circ}$, with an S-shape structure giving rise to a strong and broad dip $0-15 \operatorname{arcsec} \mathrm{W}$ of the nucleus. The residuals seem to hint for a nuclear disk as the origin of such motions (see Fig. 40). The simple rotation model used here does not account well for the data along this PA.

NGC 7328 (Fig. 41) shows regular circular gas rotation along the major axis $\left(\mathrm{PA}=88^{\circ}\right.$, both kinematical and photometric). Along the minor axis $\left(\mathrm{PA}=178^{\circ}\right)$ and at $\mathrm{PA}=118^{\circ} \mathrm{a}$ 

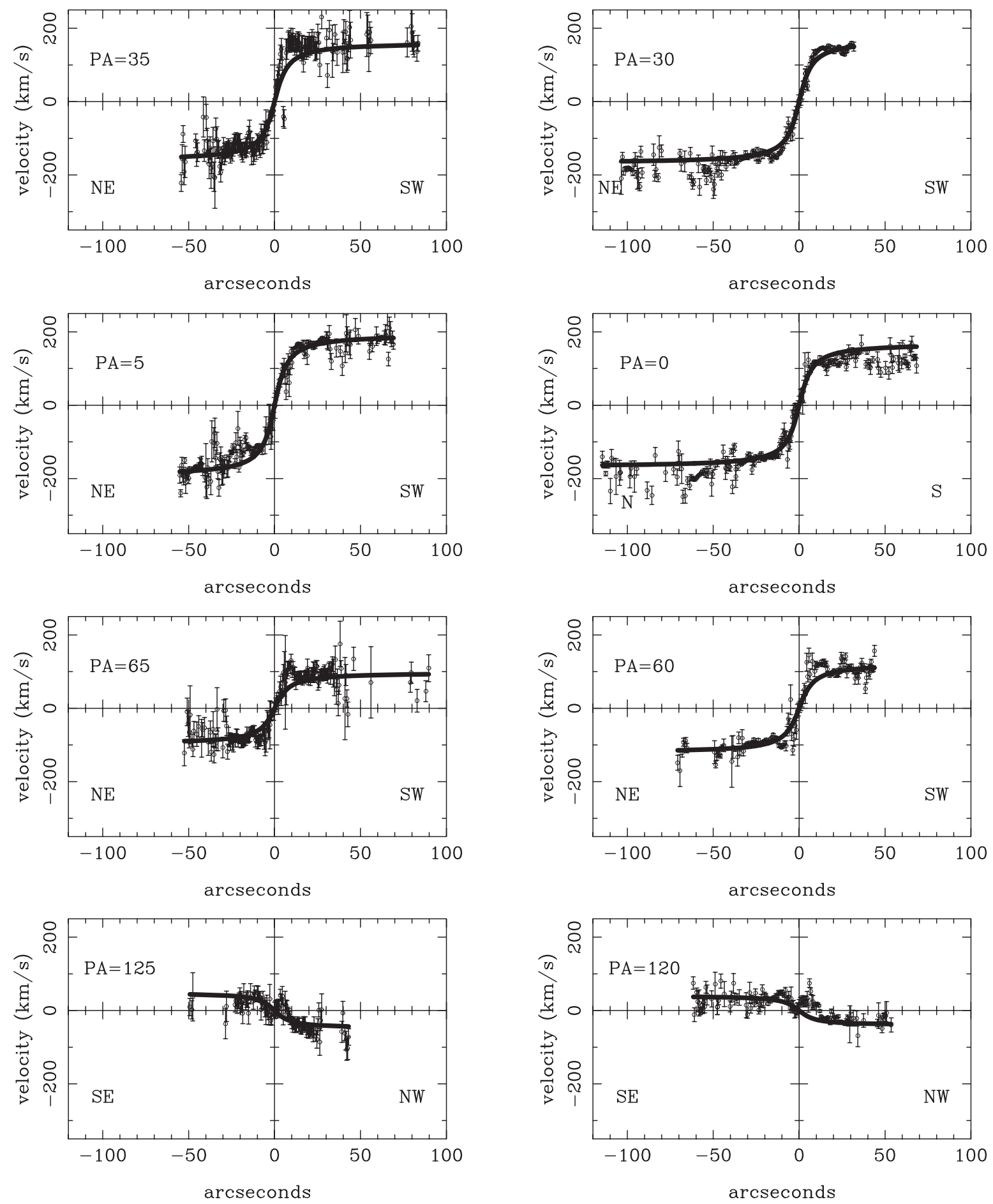

Fig. 24. Velocity distributions for ESO 139-12.

velocity dip is observed $0-12 \operatorname{arcsec} \mathrm{N}$ of the nucleus, corresponding to the most conspicuous side of an asymmetric S-like feature in the central \pm 10 arcsec. The inspection of the residuals favour the case of a nuclear disk with a minor axis close to $58^{\circ}$ (see Fig. 42 ).

\subsection{Non-active galaxies: Previous data}

The gas kinematics of five galaxies from the DEGAS control sample have previously been observed along their major axis by Márquez \& Moles (1996). These are: NGC 2712, NGC 3835, NGC 4162, NGC 4290 and NGC 6155. We made a 


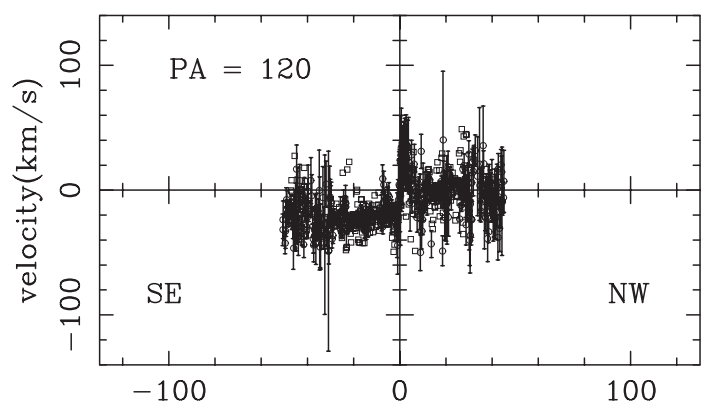

arcseconds

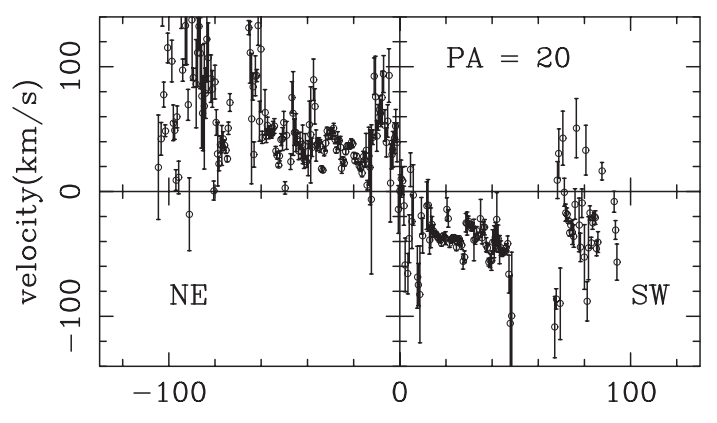

arcseconds

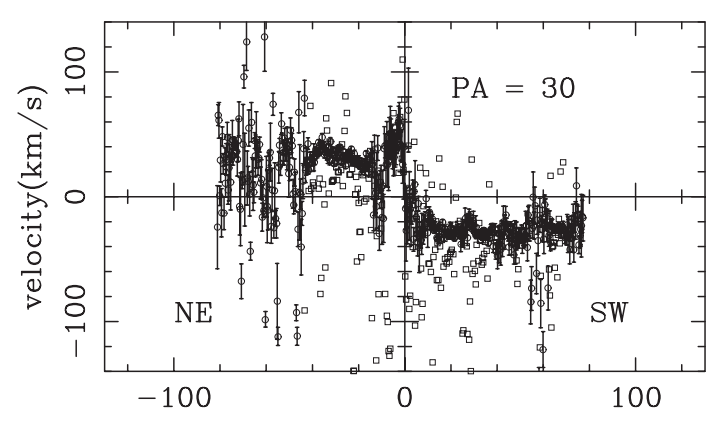

arcseconds

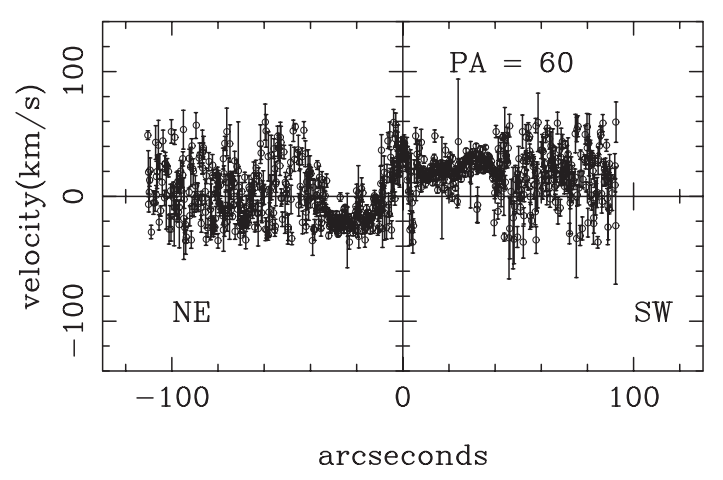

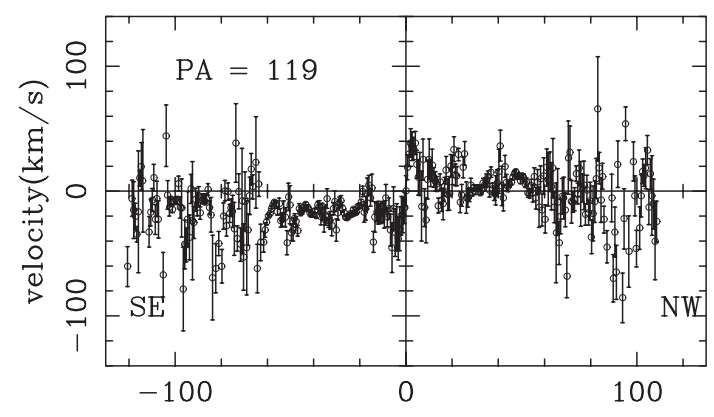

arcseconds
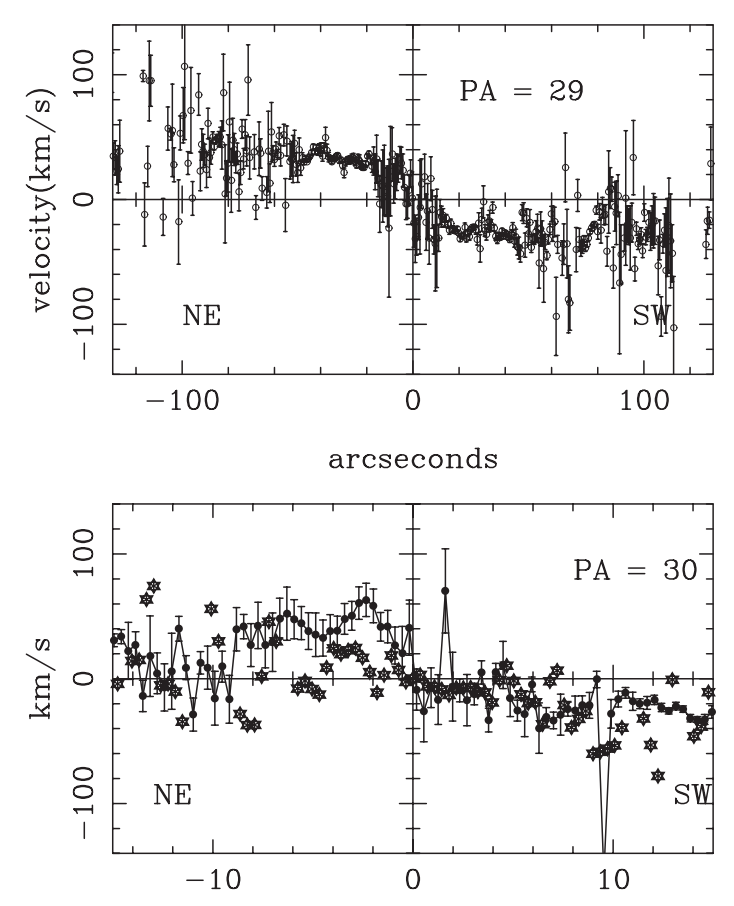

$\operatorname{arcsec}$

Fig. 26. Velocity distributions for NGC 6814 . An enlargement of the central $\pm 15 \operatorname{arcsec}$ is given for $\mathrm{PA}=30^{\circ}$ with the stellar velocity distribution as stars.

rotation model similar to that described above and give the results for these five galaxies in Table 4. Their rotation curves are given in Figs. 43-51. For NGC 2712, the rotation curve agrees with that of Héraudeau \& Simien (1998). The residuals with respect to the model are given in Figs. 44-52. In all of them evidences of the presence of S-shaped central structures can be seen. 
NGC 6860

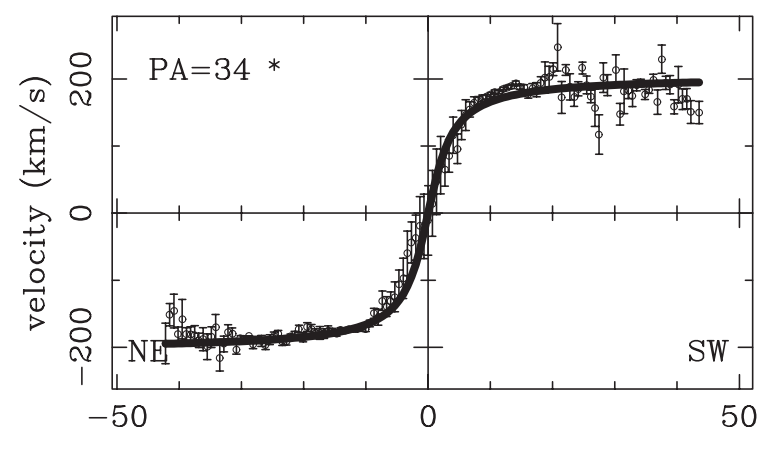

arcseconds
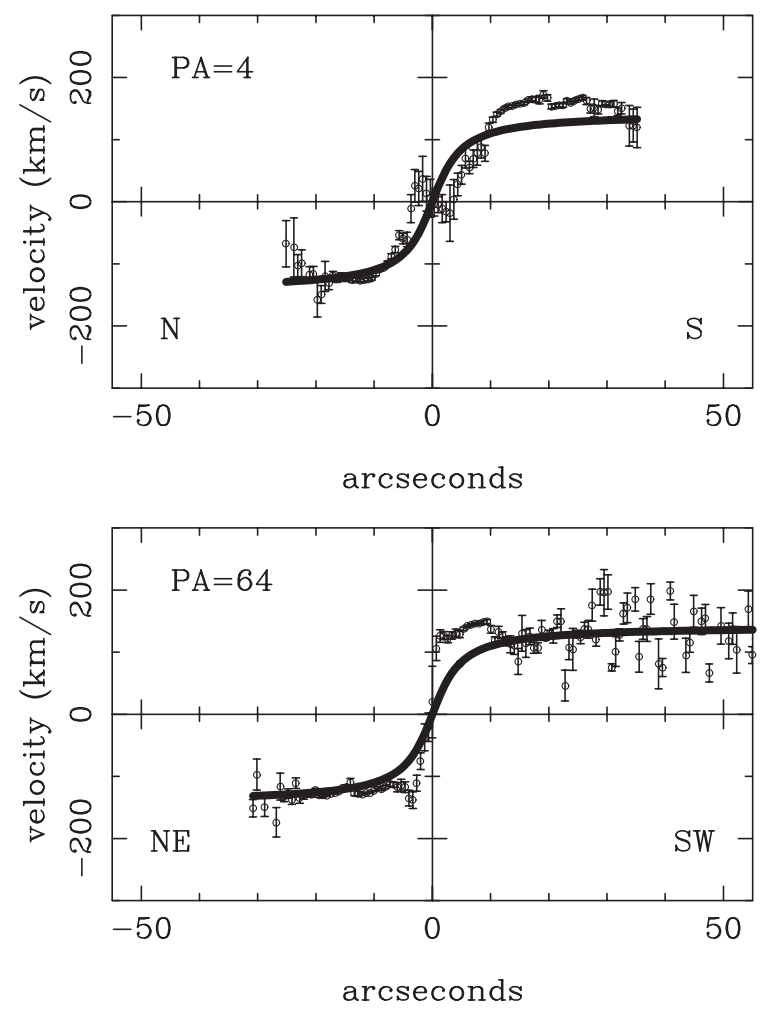

Fig. 27. Velocity distributions for NGC 6860.

\section{Nuclear and disk spectral characteristics}

The spectra for the different HII regions along the slit have been extracted and the emission lines measured as explained in M111, including only the 224 HII regions with $\mathrm{H} \alpha$ equivalent widths larger than $10 \AA$ (see below), and producing the data given in Table 5. This table is available in electronic form at the CDS via anonymous ftp to cdsarc.u-strasbg.fr $(130.79 .128 .5)$ or via http://cdsweb.u-strasbg.fr/cgi-bin/qcat?]/A+A/416/475. The columns of this table are: 1. galaxy name; 2 . slit position angle, anti-clockwise from north; 3 . distance to the nucleus along the slit in $\operatorname{arcsec} ; 4 . \mathrm{H} \alpha$ equivalent width; 5 and 6. $[\mathrm{OI}] \lambda 6300 / \mathrm{H} \alpha$ line intensity ratio and corresponding error; 7 and 8. [NII] $\lambda 6584 / \mathrm{H} \alpha$ intensity ratio and corresponding error; 9 and 10. [SII] $\lambda 6717+6731 / \mathrm{H} \alpha$ intensity ratio and

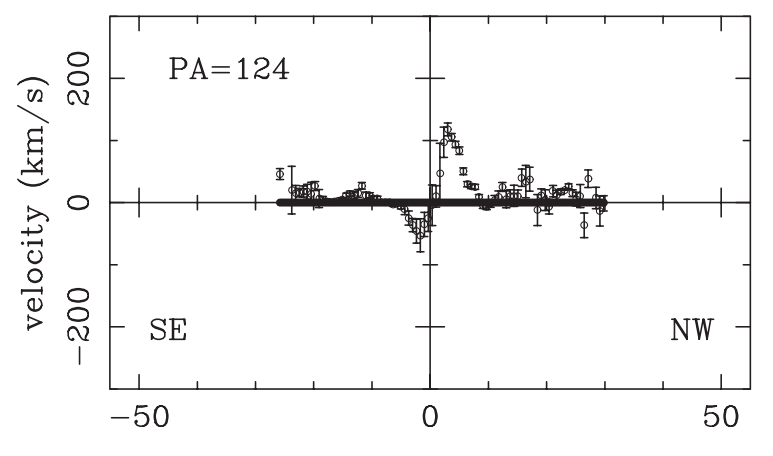

arcseconds

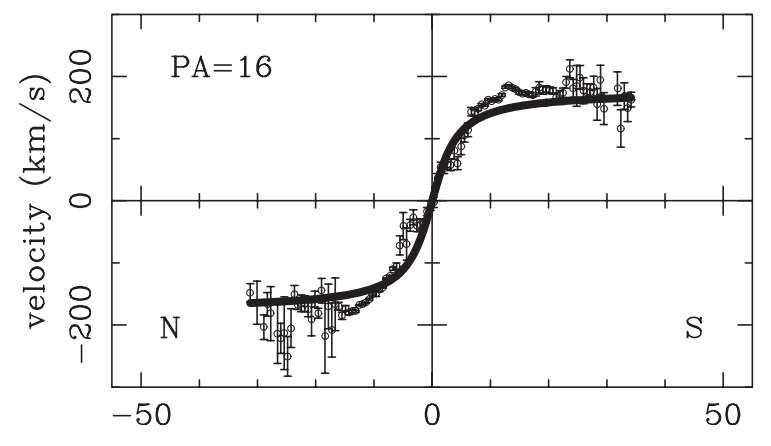

arcseconds corresponding error; 11. [SII] $] 6717 /[\mathrm{SII}] \lambda 6731$ intensity ratio.

Plots of the nuclear spectra for all the galaxies of the DEGAS sample are displayed in Figs. 53a-c. The spectra of active nuclei in general agree with the AGN classification of the Véron-Cetty \& Véron (2001) catalogue. Regarding the control galaxies, we find that 5 out of 12 have HII-like nuclei, a fraction that is lower than in M111. This could be due to the differences in morphological content between the two samples. We remember here that the control subsample was defined to match the morphological distribution of the active galaxies, which are preferentially seen in early type galaxies (Melnick et al. 1987). For the remaining 7 galaxies in the control sample, NGC 6012 has been previously classified as a LINER, and the others present a too strong $\mathrm{H} \alpha$ absorption for a reliable classification. In any case, given the strength of the [NII] lines seen 
NGC 6890
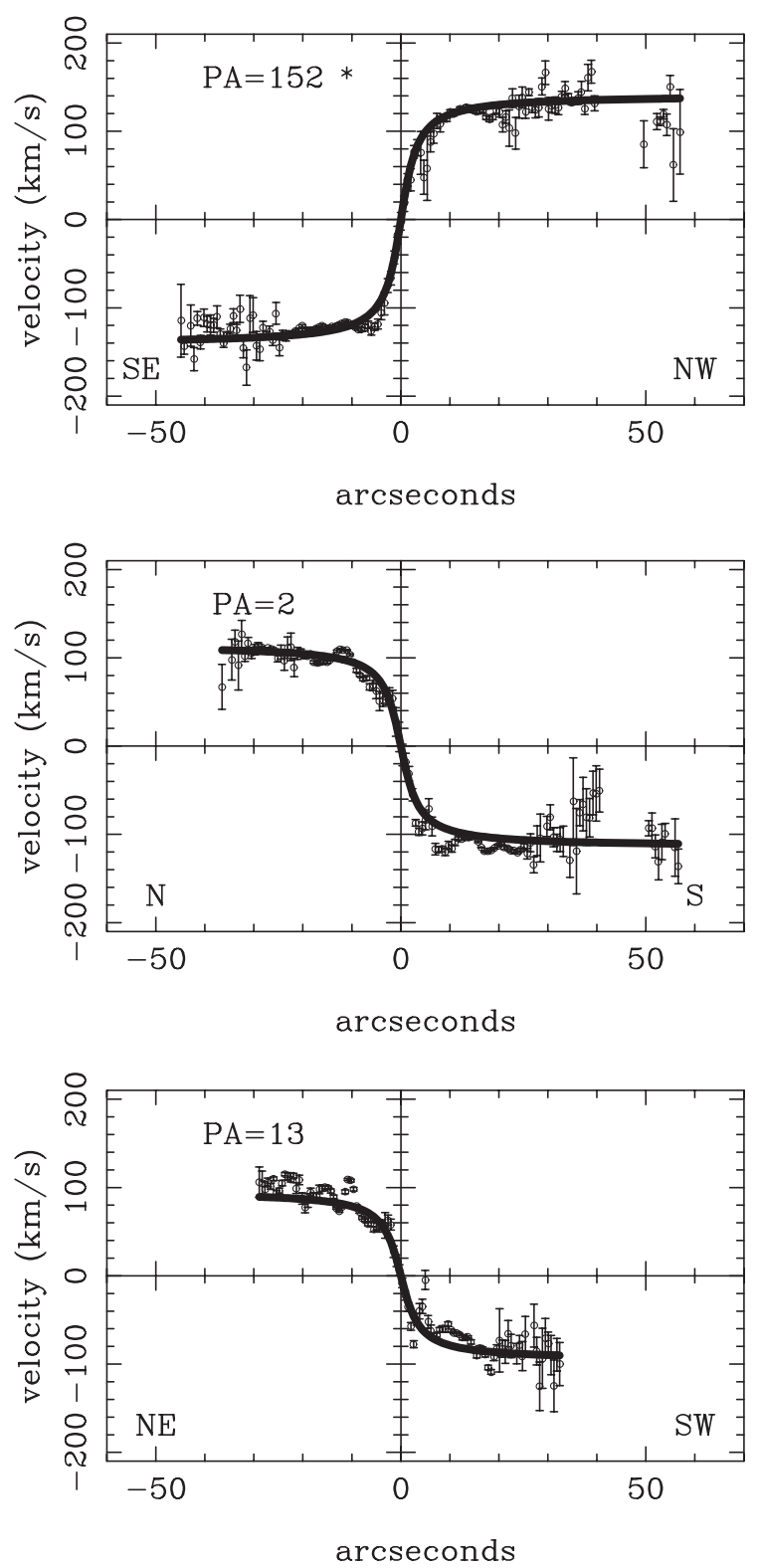

Fig. 29. Velocity distributions for NGC 6890.

in some nuclei, the possibility of some of them hosting dwarfAGN nuclei cannot be discarded. Here too, the fact that most of the galaxies are early type spirals could be relevant since, as a matter of fact, a higher percentage of low AGN activity is expected for early type spiral galaxies (Ho et al. 1997).

An estimation of the metallicities of the disk HII regions and their possible gradients were obtained from the $[\mathrm{NII}] / \mathrm{H} \alpha$ line ratios only. Denicoló et al. (2002) obtained an empirical calibration of the oxygen abundances based on this ratio, which they showed to be powerful when analyzing large survey data to rank their metallicities, but with quite large uncertainties on individual objects, mainly due to $\mathrm{O} / \mathrm{N}$ abundance ratio and ionization degree variations. Here we only consider it to study global trends of $Z$ from the spectra of HII regions
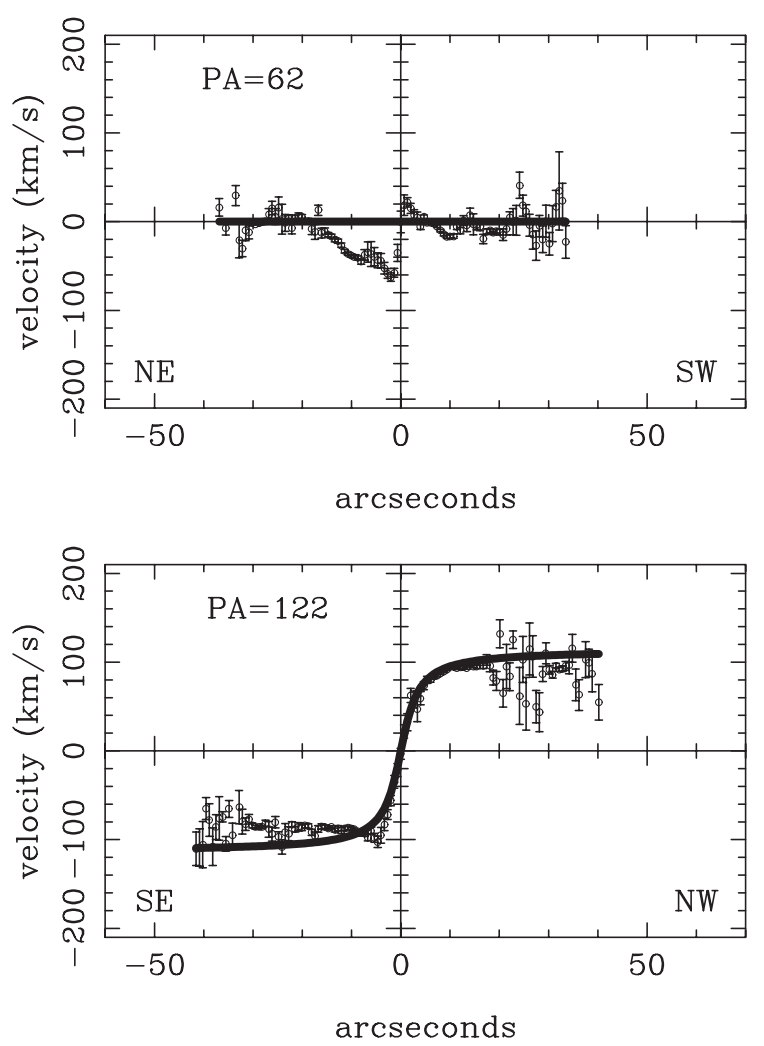

arcseconds in our sample ${ }^{5}$, along the same lines discussed in M111. The data don't allow us to conclude on each individual galaxy, but can be used to look for general trends when the population of the disk HII regions is considered as a whole. To be able to combine data from different galaxies, we have normalized the galactocentric distances of the HII regions to $r_{25}$ (as given in the $\mathrm{RC} 3)^{6}$. A plot of the $[\mathrm{NII}] / \mathrm{H} \alpha$ ratios as a function of distance to the galaxy nucleus is displayed in Fig. 54 for the DEGAS sample, together with the values taken from the

\footnotetext{
${ }^{5}$ Note that we do not refer to the regions very close to the AGN in active galaxies.

${ }^{6}$ In the analysis we only include $\mathrm{HII}$ regions with $\mathrm{H} \alpha$ equivalent widths larger than $10 \AA$, as in M111. In that way we avoid including regions with strong Balmer absorption, that could induce inconsistencies in the estimation of the metallicity.
} 
NGC 151
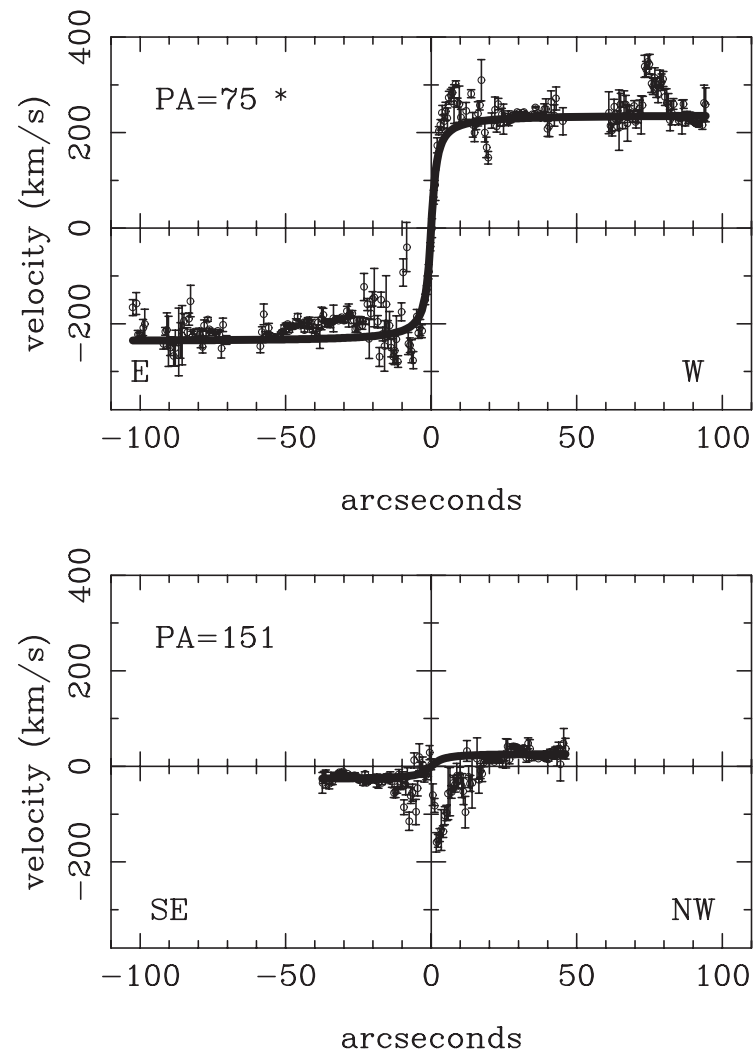

Fig. 31. Velocity distributions for NGC 151.

NGC 1357

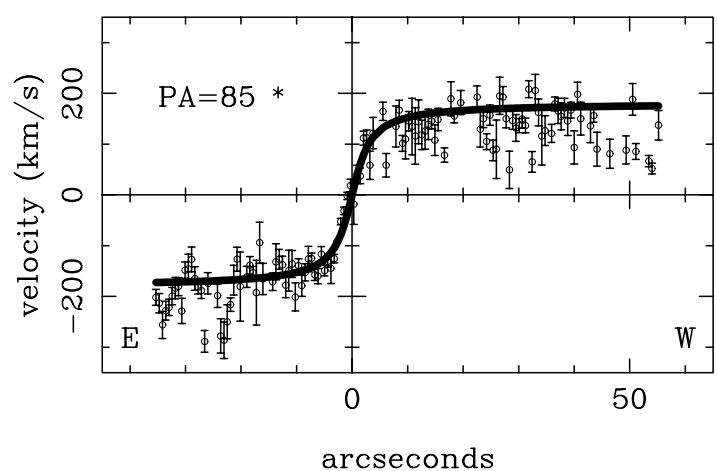

Fig. 33. Velocity distribution for NGC 1357.

isolated spirals (24 galaxies) of the M111 sample. It should be noticed that even if the total number of HII regions involved is large (224), they correspond to 15 active galaxies and 10 non-active ones, with unequal weighting for the number of position angles per galaxy. In order to give the same weight to each galaxy, independently of the number of position angles observed, we consider hereafter only the HII regions along the major axes, in such a way that they can be directly compared to the isolated galaxies in M111. Our main result is that disk metallicities of active and control galaxies are the same, since

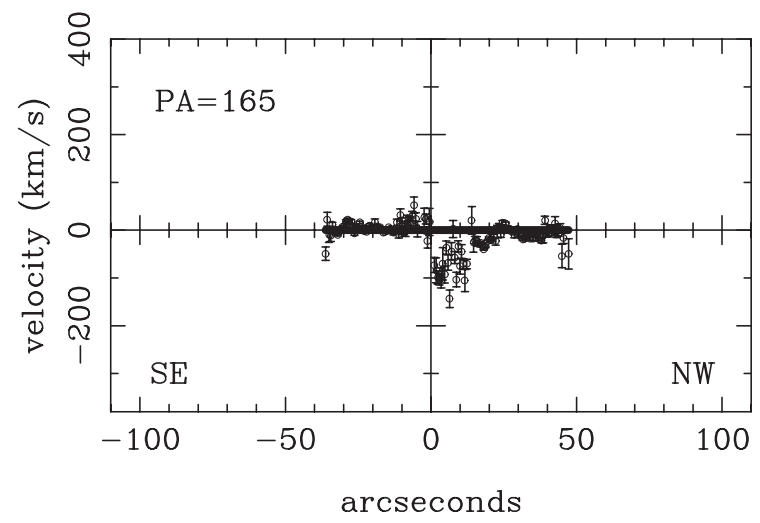

the differences are found only at a $1 \sigma$ level: the median values are $[\mathrm{NII}] / \mathrm{H} \alpha=0.46 \pm 0.07$ for active galaxies and $0.39 \pm 0.07$ for non-active ones, to be compared with $[\mathrm{NII}] / \mathrm{H} \alpha=0.31 \pm$ 0.07 for the isolated galaxies in M111 covering the same absolute magnitude range, i.e., $M_{B}$ between -19 and -22 .

We find that there is a deficiency of low [NII]/H $\alpha$ values for the two DEGAS subsamples. If we now consider two families per subsample, early (Sa to Sb) and late (Sbc to Scd) spirals, we see that the deficiency is specially clear for late types. However, the DEGAS sample is obviously biased towards early type spirals in the case of active galaxies (see for instance Moles et al. 1995) and by construction for the control sample, and therefore no firm conclusions can be reached.

\section{Summary and conclusions}

We have presented and discussed the properties derived from the analysis of long slit spectra along several position angles of a sample of isolated active and non-active galaxies. A simple model has been used to trace overall rotation curve properties and obtain the parameters for Tully-Fisher (TF) analysis. The active and non-active spirals define very similar TF relations, populating the region occupied by isolated spirals of early types. In general, the rotation curves broadly agree with circular rotation, but the pure rotation model fails to account 
IC 454
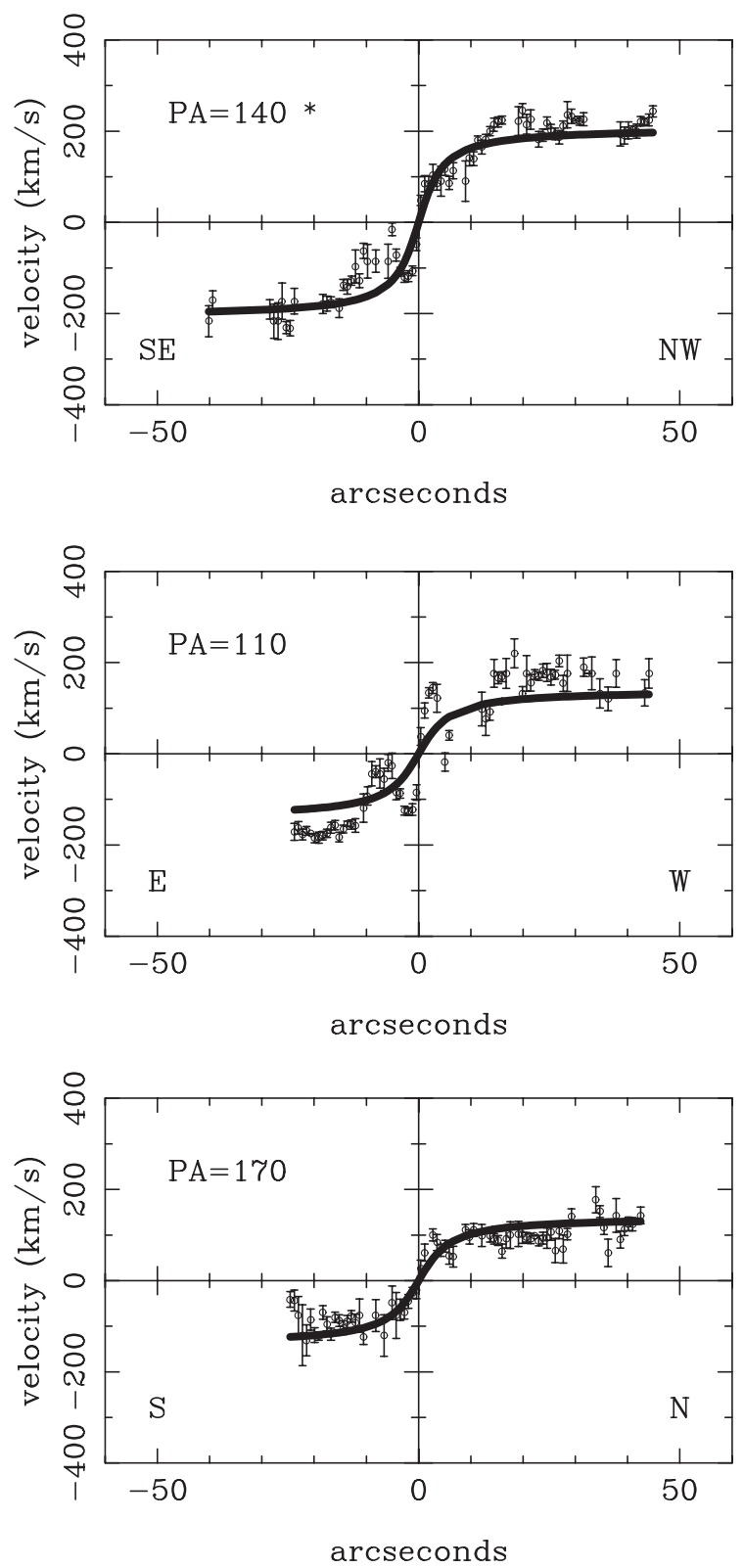
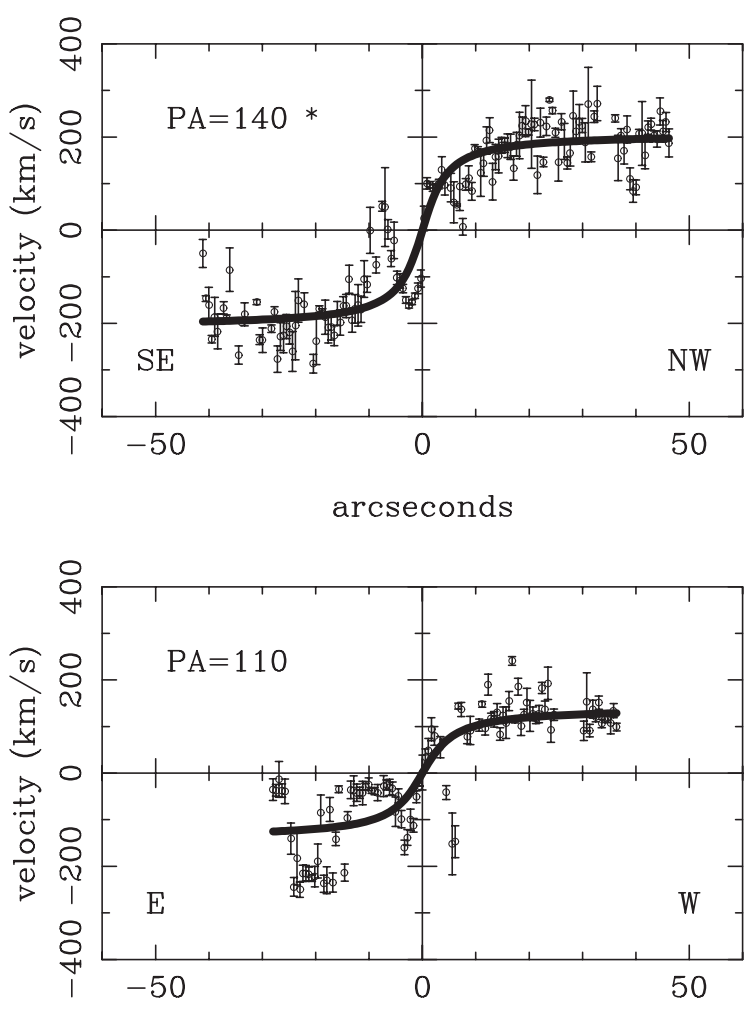

arcseconds

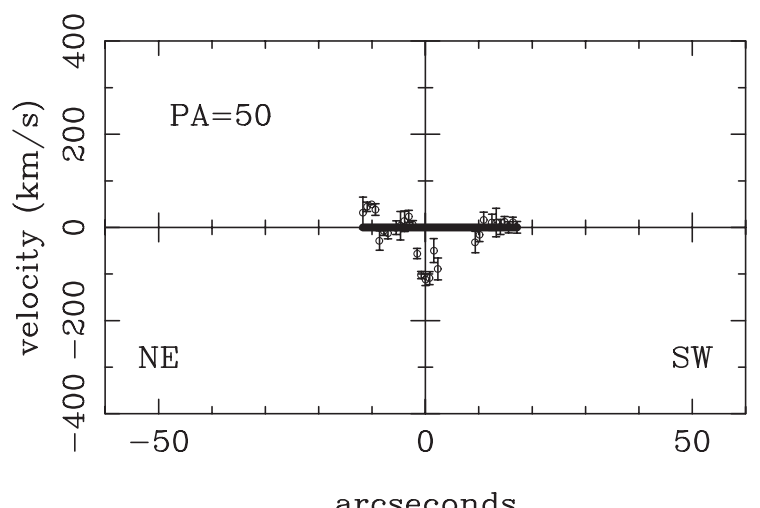

Fig. 35. Velocity distributions for IC 454 . The two sets of curves along PA $=140^{\circ}$ and $\mathrm{PA}=110^{\circ}$ correspond to ESO (left) and CAHA (right) data.

NGC 3571

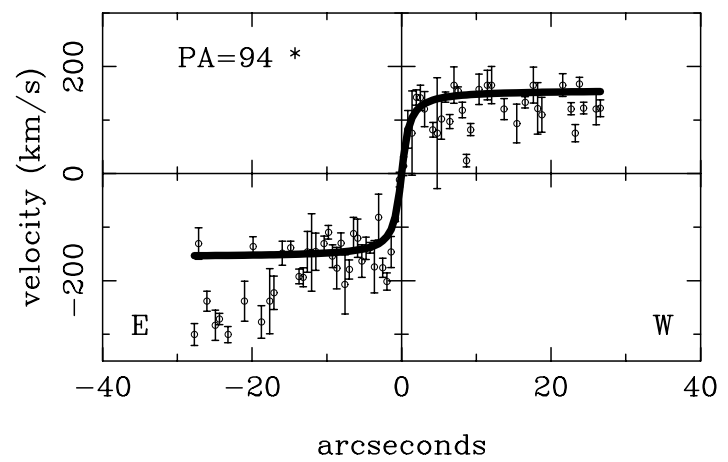

for large scale velocity distribution peculiarities, that can be attributed to the presence of a large scale bar, spiral-arm components, or small-scale central deviations indicative of the presence of non-circular motions and/or decoupled disks. Note that the same kind of small scale central S-shaped deviations from the rotation curve model are found in both active (whether they have a single or double barred host, or are of Sy 1 or Sy 2 type) and non-active galaxies. When enough position angles are available, a better indication can be obtained of whether an inner bar (most cases) or a nuclear disk (see IC 184, NGC 3660, NGC 5728, ESO 139 and NGC 6012) are at the origin of such features.

Fig. 37. Velocity distributions for NGC 3571. 
NGC 6012

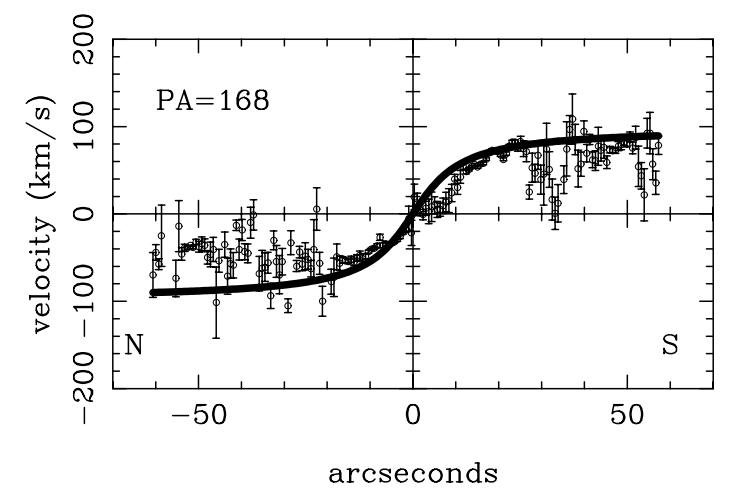

Fig. 39. Velocity distributions for NGC 6012.

NGC 7328

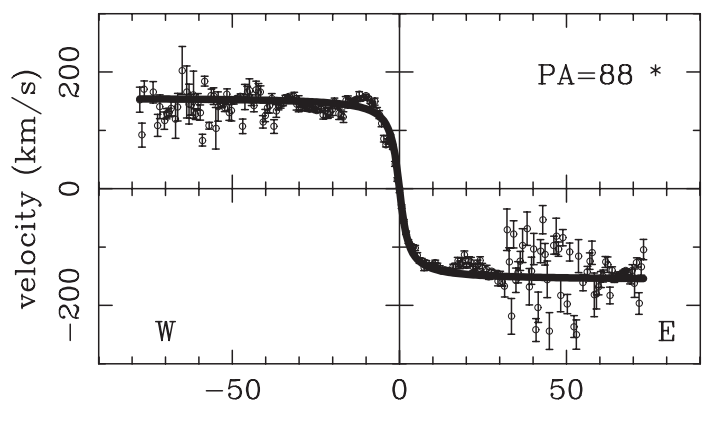

arcseconds

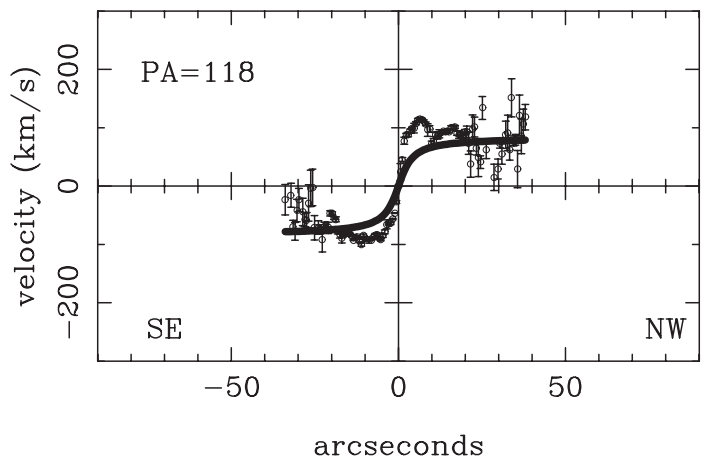

Fig. 41. Velocity distributions for NGC 7328.

NGC 2712

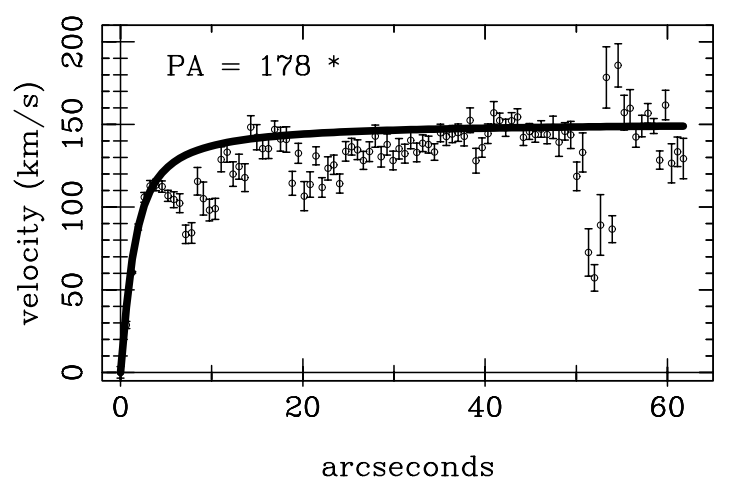

Fig. 43. Average velocity distribution for NGC 2712.
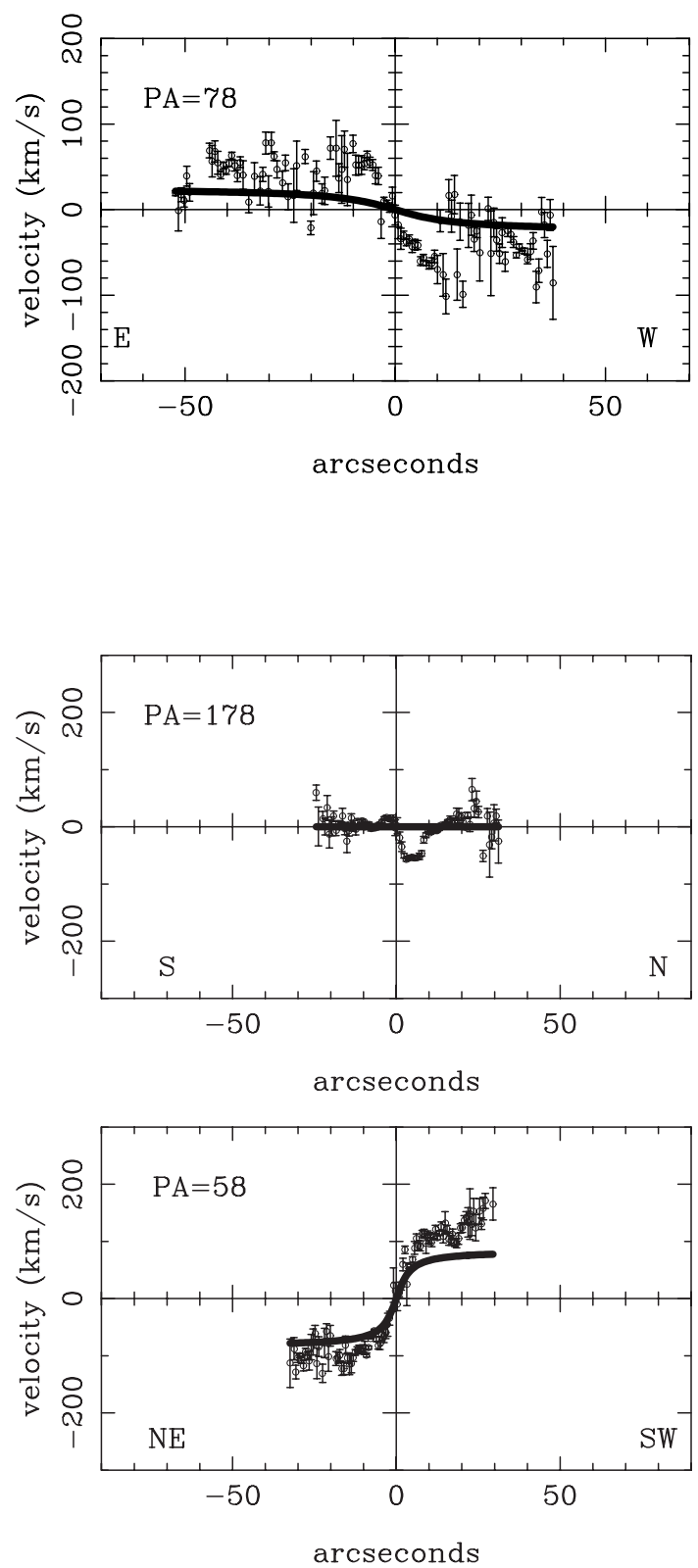

NGC 3835

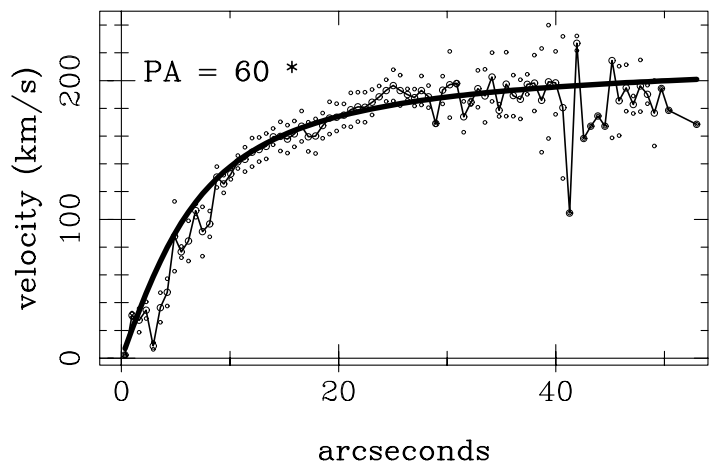

Fig. 45. Average velocity distribution for NGC 3835 . The individual points correspond to the data on either side of the nucleus. 
NGC 4162

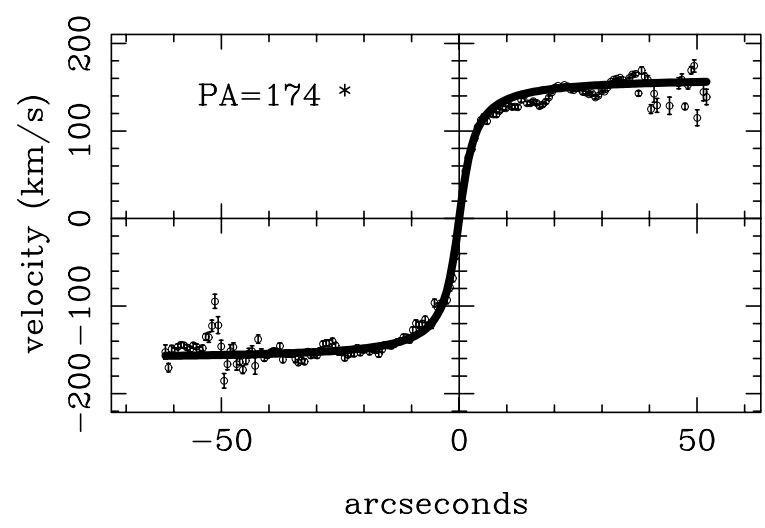

Fig. 47. Velocity distribution for NGC 4162.

NGC 4290

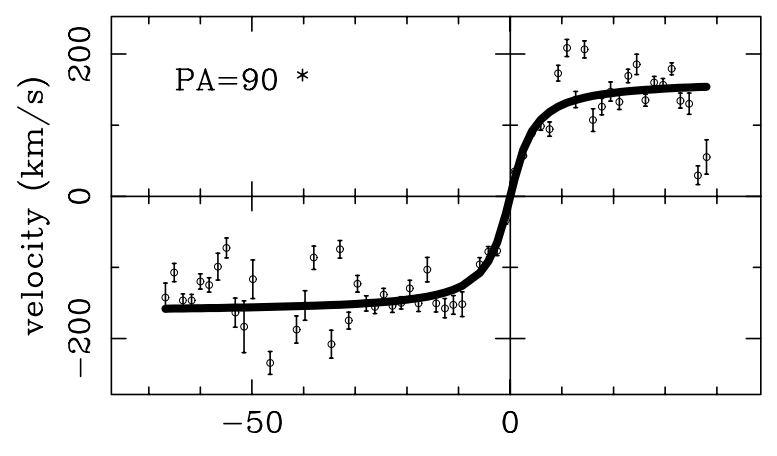

arcseconds

Fig. 49. Velocity distribution for NGC 4290.

NGC 6155

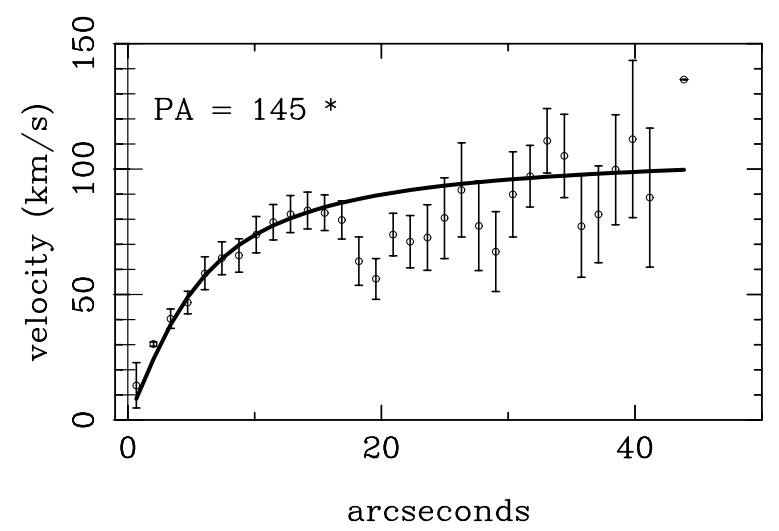

Fig. 51. Average velocity distribution for NGC 6155.
Disk HII region metallicities, estimated from the $[\mathrm{NII}] / \mathrm{H} \alpha$ ratios, are also very similar for the active and non-active sub-samples.

The analysis of the photometric properties of the DEGAS sample obtained from NIR imaging has already shown that active and non-active isolated spiral galaxies share the same disk, bulge and primary bar properties, and that they are indistinguishable at least down to spatial scales of about $300 \mathrm{pc}$ (Márquez et al. 1999, 2000). These results are reinforced here, in the sense that both kinematical properties and disk metallicities lead to the same conclusion. Thus, whether or not they have an AGN, the spiral galaxies in the DEGAS sample are equivalent in all respects (a general discussion based on the DEGAS data will be presented in a forthcoming paper). Therefore, the differences claimed to be related to the mechanisms driving AGN activity should be found in details related to circumnuclear regions still unresolved in our observations. But, interestingly, no significant morphological differences were found, even in the innermost few arcseconds, for a sample of $123 \mathrm{ac}-$ tive and non-active galaxies imaged with the HST (Martini et al. 2003). Besides, the AGN activity can involve such different time scales from those of the dynamical processes under study, that any direct connection would be out of scope (see e.g. Hunt \& Malkan 1999). Finally, and considering the growing evidence that all galaxies above a certain luminosity host central, massive compact objects (McLure \& Dunlop 2002), our results are fully compatible with the idea that the AGN activity could take place recurrently in a given galaxy; in this way, the AGN itself would be seen when fuel is made available in the most internal regions. Note however that our sample is small and that differences between the active and non-active samples may be lost due to the poor statistics; in the recent works by the SLOAN project, for example, several thousand galaxies were required to statistically identify clear differences between active and non active galaxies (Kauffmann et al. 2003).

Acknowledgements. I.M. acknowledges financial support from the IAA and the Spanish Ministerio de Ciencia y Tecnología through a Ramón y Cajal fellowship. This work is financed by DGICyT grants PB93-0139, PB96-0921, PB98-0521, PB98-0684, ESP98-1351, AYA2001-2089, AYA2001-3939-C0301 and the Junta de Andalucía grant TIC-144. We acknowledge financial support from the Picasso Programme d'Actions Integrées of the French and Spanish Ministries of Foreign Affairs. F.D. acknowledges financial support from CNRS-INSU for several observing trips. J. Maza gratefully acknowledges support from the Chilean Centro de Astrofísica FONDAP 15010003 . We thank Ignacio Marrero for his collaboration in some data acquisition and calibration. This research has made use of the NASA/IPAC extragalactic database (NED), which is operated by the Jet Propulsion Laboratory under contract with the National Aeronautics and Space Administration. Finally, we are grateful to the referee for a number of interesting comments that helped us improve the presentation. 
UGC1395

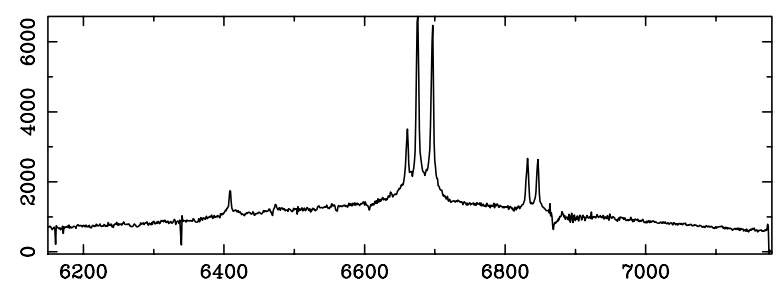

IC1816

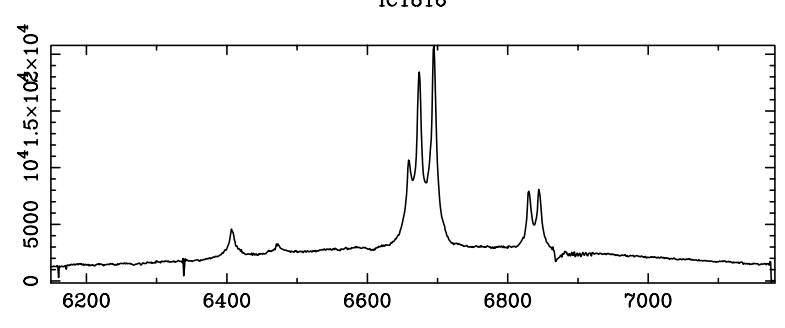

NGC2639

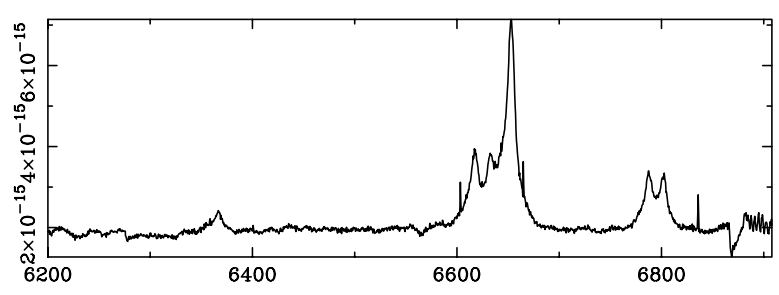

NGC3660

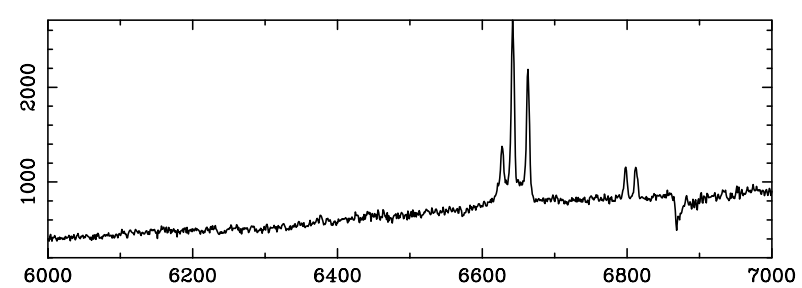

NGC4785

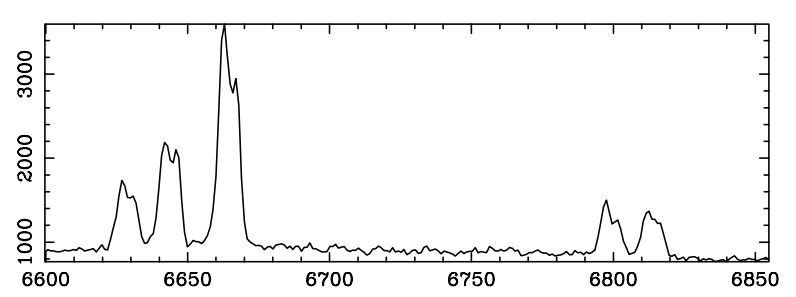

NGC5728

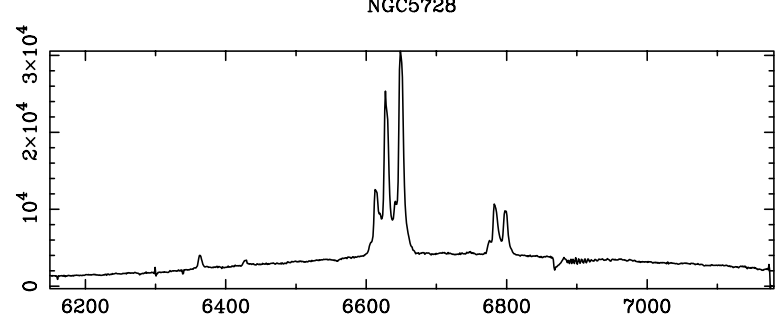

IC184

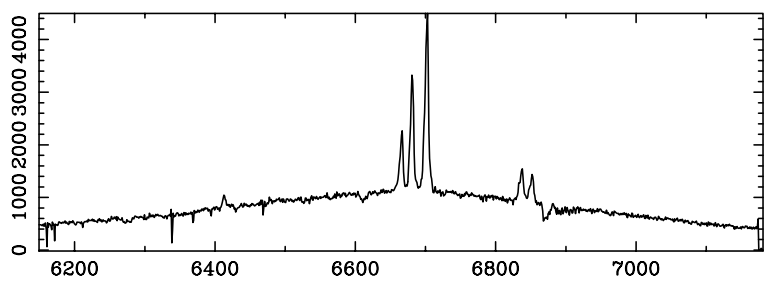

UGC3223

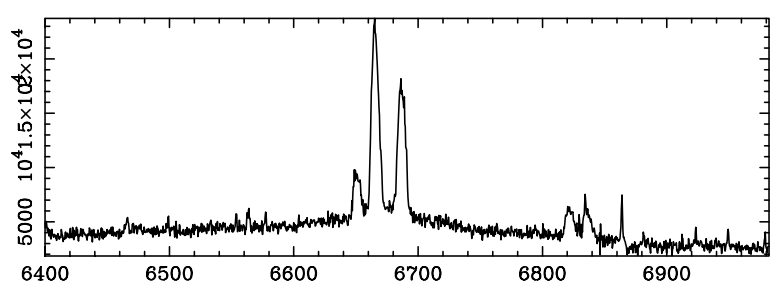

IC2510

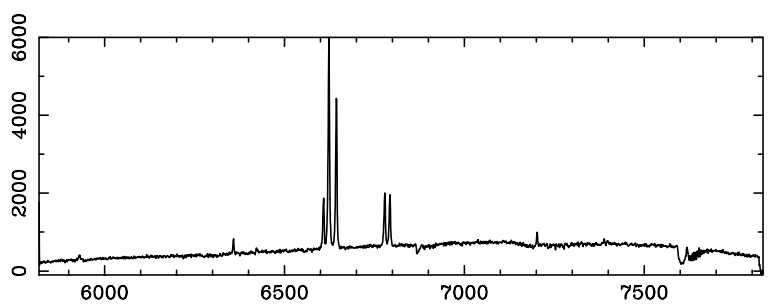

NGC4507

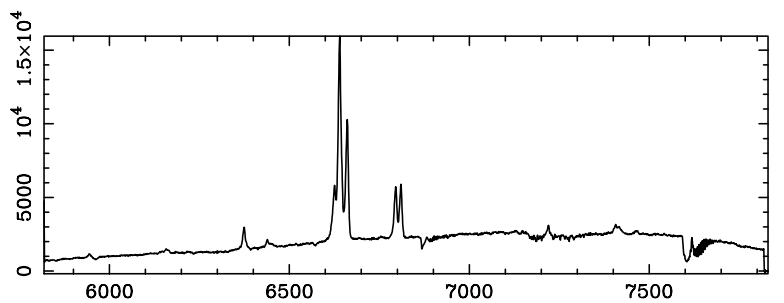

NGC5347

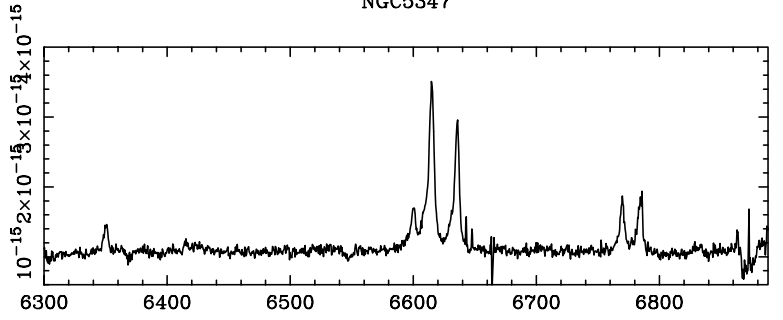

ESO139---

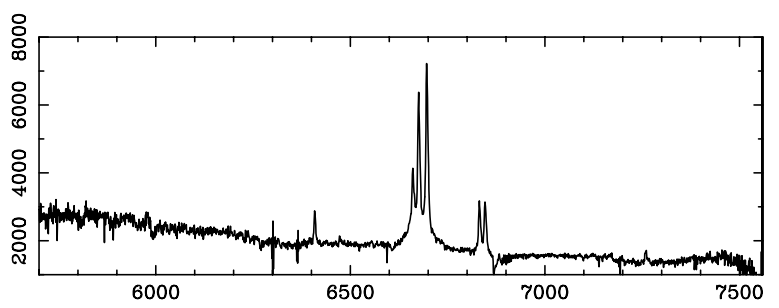

Fig. 53. a. Nuclear spectra, plotted as non calibrated flux vs. observed wavelength (in $\AA$ ), except for NGC 2639 and NGC 5347 which are flux calibrated (in erg s${ }^{-1} \mathrm{~cm}^{-2} \AA^{-1}$ ). 
NGC6814

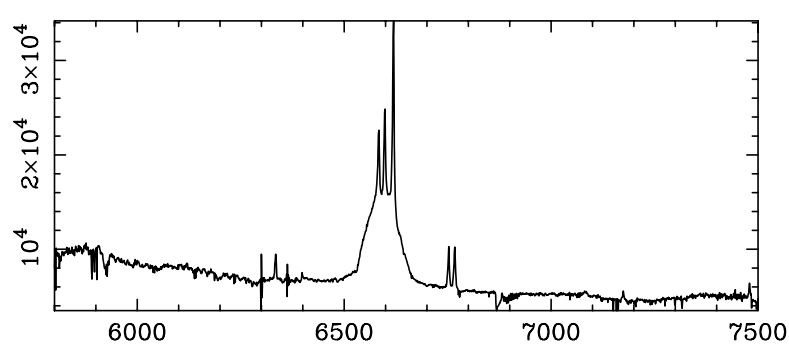

NGC6890

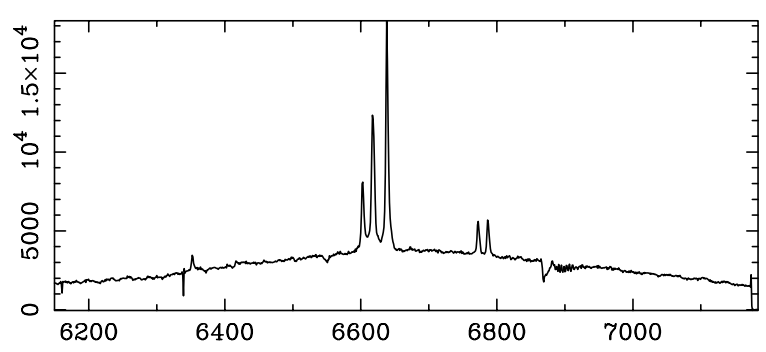

NGC1357

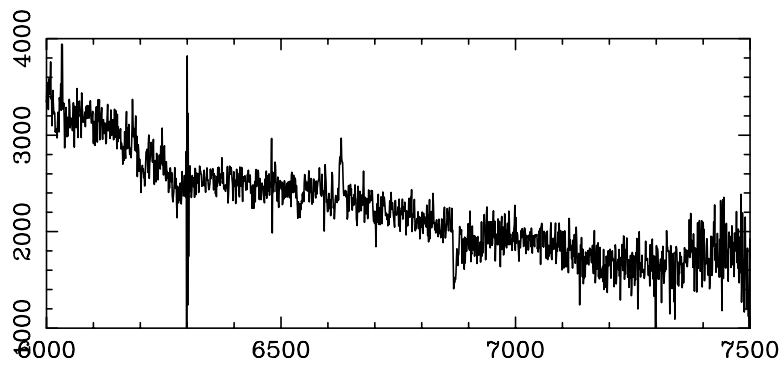

NGC2811

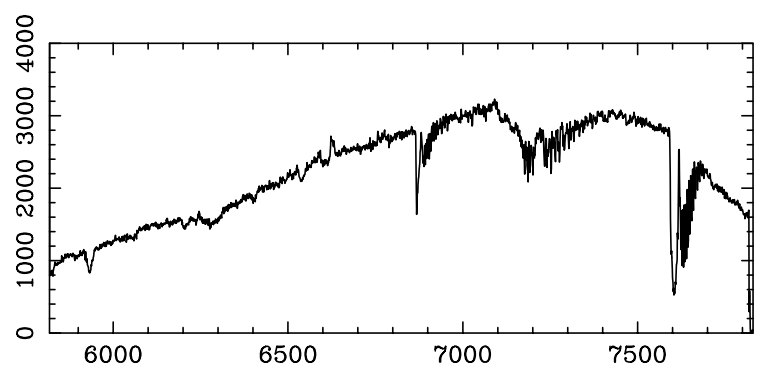

NGC6012

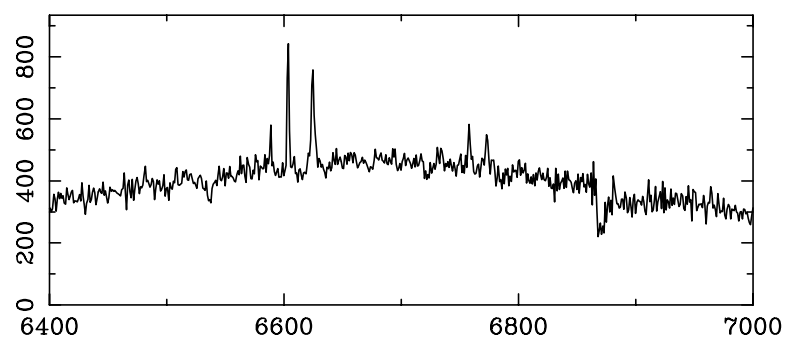

NGC6860

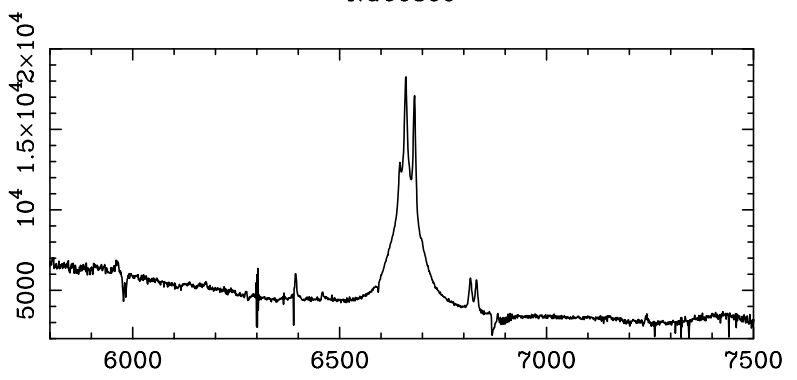

NGC151

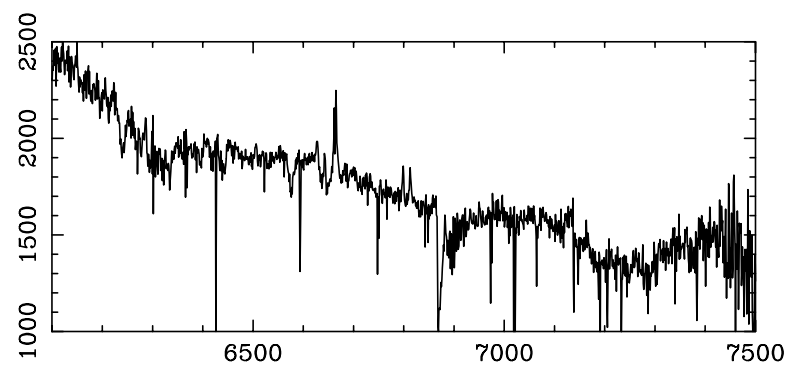

IC454

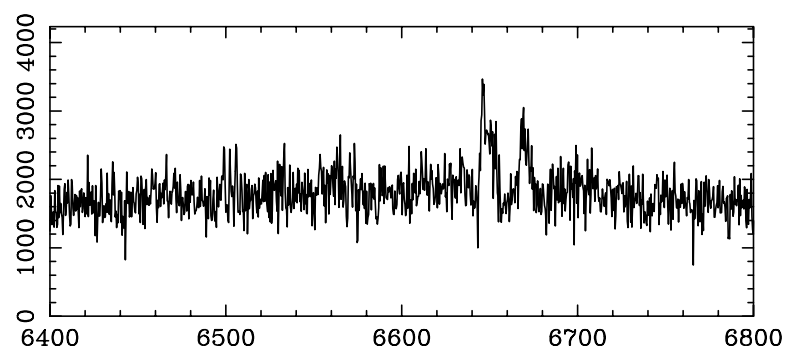

NGC3571

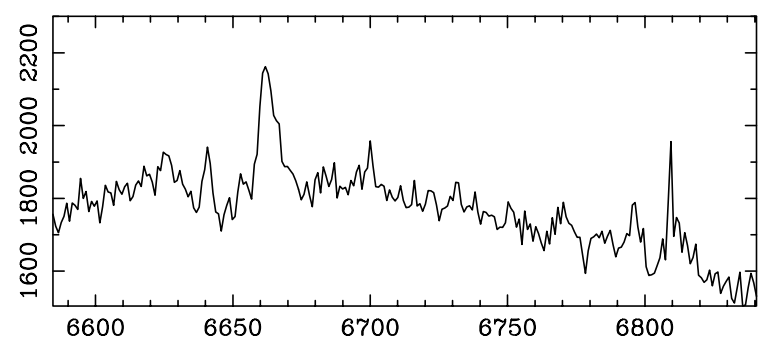

NGC7328

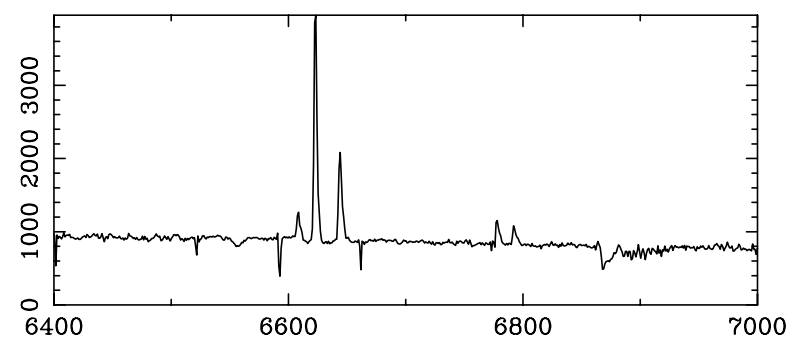

Fig. 53. b. Nuclear spectra, plotted as non calibrated flux vs. observed wavelength (in $\AA$ ). 

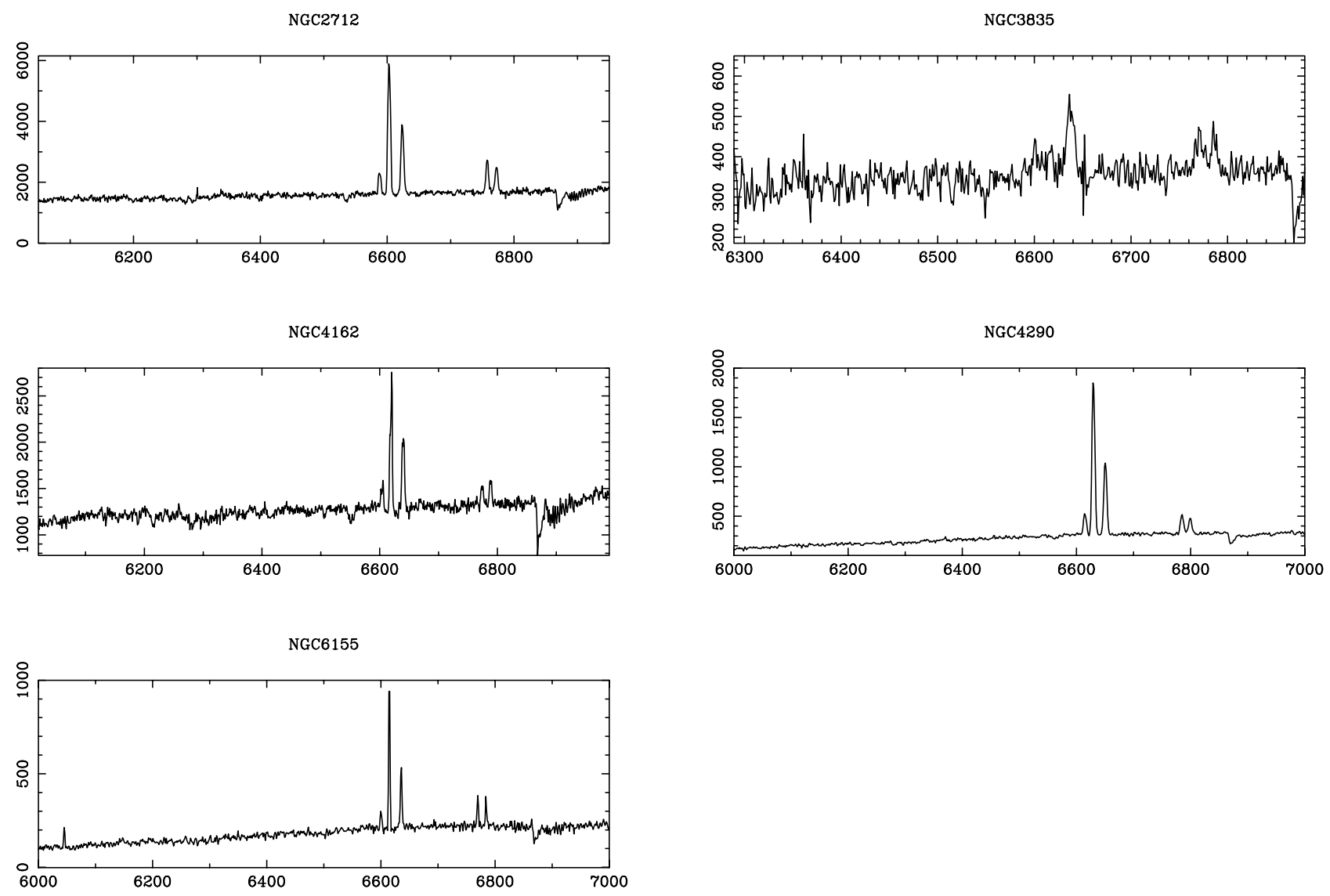

Fig. 53. c. Nuclear spectra, plotted as non calibrated flux vs. observed wavelength (in $\AA$ ).

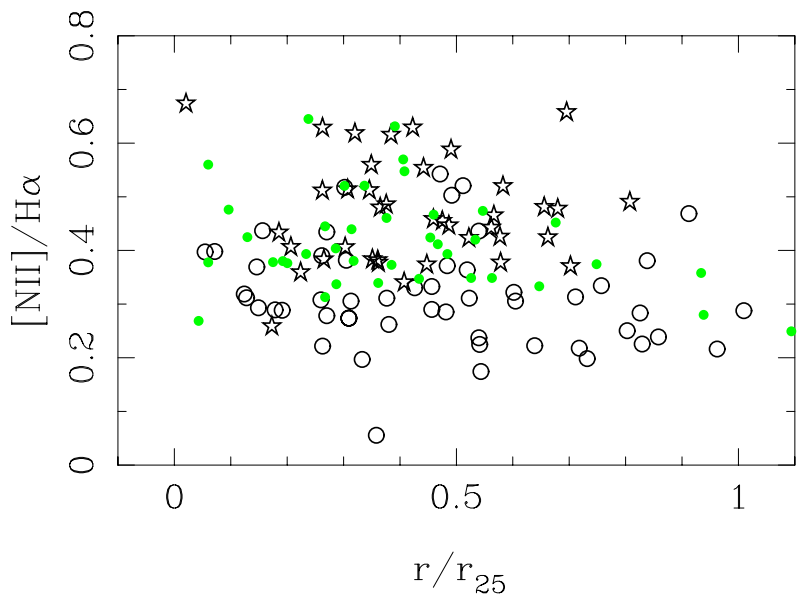

Fig. 54. $[\mathrm{NII}] / \mathrm{H} \alpha$ intensity ratio as a function of distance of HII region to the nucleus, normalized to $r_{25}$ for the DEGAS sample (with stars for active galaxies and filled circles for non-active objects) and for the isolated spirals in M111 (empty circles) having the same absolute magnitude distribution $\left(-22<M_{B}<-19\right)$ (see text).

\section{References}

Courteau, S. 1997, AJ, 114, 2402

Emsellem, E., Greusard, D., Combes, F., et al. 2001, A\&A, 368, 52 Héraudeau, P., \& Simien, F. 1998, A\&AS, 133, 317

Ho, L. C., Filippenko, A. V., \& Sargent, W. L. W. 1997, ApJS, 112, 315
Hunt, L. K., \& Malkan, M. A. 1999, ApJ, 516, 660

Kauffmann, G., Heckman, T. M., Tremonti, C., et al. 2003, MNRAS, 346,1055

Kollatschny, W., \& Fricke, K. J. 1987, A\&A, 183, 9

Márquez, I., \& Moles, M. 1996, A\&AS, 120, 1

Márquez, I., Durret, F., González Delgado, R. M., et al. 1999, A\&AS, 140,1

Márquez, I., Durret, F., Masegosa, J., et al. 2000, A\&A, 360, 431

Márquez, I., Masegosa, J., Moles, M., et al. 2002, A\&A, 393, 389

Márquez, I., Masegosa, J., Durret, F., et al. 2003, A\&A, 409, 459

Martini, P., Regan, M. W., Mulchaey, J. S., \& Pogge, R. W. 2003, ApJ, 589,774

McLure, R. J., \& Dunlop, J. S. 2002, MNRAS, 331, 795

Melnick, J., Terlevich, R., \& Moles, M. 1986, in Luminous Stars and Associations in Galaxies, ed. C. W. H. De Loore, A. Wilkis, \& P. Laskarides (Dordrecht: Reidel), 505

Moles, M., Márquez, I., \& Pérez, E. 1995, ApJ, 438, 604

Pérez, E., Márquez, I., Marrero, I., et al. 2000, A\&A, 353, 893

Persic, M., \& Salucci, P. 1995, ApJS, 99, 501

Pogge, R. W., \& Martini, P. 2002, ApJ, 569, 624

Prada, F., \& Gutiérrez, C. M. 1999, ApJ, 517, 123

Schommer, R. A., Caldwell, N., Wilson, A. S., et al. 1988, ApJ, 324, 154

de Vaucouleurs, G., de Vaucouleurs, A., Corwin, H. G. Jr, et al. 1991, Third Reference Catalogue of Bright Galaxies, XII (1-3), 2069 (Springer-Verlag) (RC3)

Véron-Cetty, M. P., \& Véron, P. 2001, A\&A, 375, 791 


\section{Online Material}


I. Márquez et al.: Long slit spectroscopy of spirals with and without AGN, Online Material p 2

Table 3. Detailed log of the spectra.

\begin{tabular}{|c|c|c|c|c|c|c|c|}
\hline $\begin{array}{l}\text { Galaxy } \\
\text { (Active) }\end{array}$ & $\overline{\text { Run }}$ & $\begin{array}{l}\mathrm{PA} \\
\left({ }^{\circ}\right)\end{array}$ & $\begin{array}{l}\text { Exp. Time } \\
\text { (s) }\end{array}$ & $\begin{array}{l}\text { Galaxy } \\
\text { (Non-active) }\end{array}$ & $\overline{\text { Run }}$ & $\begin{array}{l}\mathrm{PA} \\
\left({ }^{\circ}\right)\end{array}$ & $\begin{array}{l}\text { Exp. Time } \\
\text { (s) }\end{array}$ \\
\hline \multirow[t]{6}{*}{ UGC 1395} & WHT 96 & 35 & 1800,1800 & NGC 151 & LC 898 & 75 & 2400 \\
\hline & & 125 & 0,3600 & & & 151 & 2400 \\
\hline & WHT 99 & 80 & 3600,1800 & & & 165 & 2400 \\
\hline & LC 896 & 135 & 3600 & NGC 1357 & LC 898 & 85 & 1860 \\
\hline & LC 898 & 45 & 2400 & IC 454 & CAHA 00 & 110 & 3600,1800 \\
\hline & LC 898 & 165 & 2400 & & & 140 & 3600,1800 \\
\hline \multirow[t]{6}{*}{ IC 184} & WHT 99 & 7 & 3600,3600 & & ESO 96 & 50 & 3600 \\
\hline & & 97 & 3600,1800 & & & 110 & 3600 \\
\hline & LC 896 & 7 & 3600 & & & 140 & 3600 \\
\hline & & 37 & 2400 & & & 170 & 3000 \\
\hline & & 97 & 3600 & NGC 2811 & ESO 96 & 20 & 3000 \\
\hline & & 157 & 2400 & & & 50 & 2400 \\
\hline \multirow[t]{2}{*}{ IC 1816} & LC 896 & 0 & 2400 & & & 110 & 3600 \\
\hline & & 90 & 2400 & & & 170 & 2400 \\
\hline \multirow[t]{7}{*}{ UGC 3223} & WHT 99 & 80 & 3600,1800 & NGC 3571 & LC 596 & 94 & 3600 \\
\hline & ESO 96 & 50 & 3600 & & & 64 & 3600 \\
\hline & & 80 & 3600 & & & 4 & 3600 \\
\hline & & 110 & 2400 & NGC 6012 & LC 896 & 78 & 2400 \\
\hline & & 170 & 3600 & & & 168 & 2400 \\
\hline & CAHA 00 & 20 & 3600,3600 & NGC 7328 & LC 896 & 88 & 2400 \\
\hline & & 170 & 3600,1800 & & & 178 & 2400 \\
\hline \multirow[t]{3}{*}{ NGC 2639} & WHT 99 & 0 & 1800,1800 & & LC 898 & 58 & 3600 \\
\hline & & 45 & 3600,1800 & & & 118 & 2400 \\
\hline & & 315 & 3600,1800 & & & & \\
\hline \multirow[t]{4}{*}{ IC 2510} & ESO 96 & 58 & 3600 & & & & \\
\hline & & 118 & 3600 & & & & \\
\hline & & 148 & 3600 & & & & \\
\hline & & 178 & 3000 & & & & \\
\hline \multirow[t]{7}{*}{ NGC 3660} & WHT 99 & 115 & 3600,1800 & & & & \\
\hline & ESO 96 & 25 & 3600 & & & & \\
\hline & & 85 & 2400 & & & & \\
\hline & & 115 & 3600 & & & & \\
\hline & & 145 & 2400 & & & & \\
\hline & CAHA 00 & 25 & 3600,1800 & & & & \\
\hline & WHT 00 & 85 & 3600,1800 & & & & \\
\hline \multirow[t]{2}{*}{ NGC 4507} & ESO 96 & 51 & 3600 & & & & \\
\hline & & 141 & 3340 & & & & \\
\hline \multirow[t]{2}{*}{ NGC 4785} & LC 596 & 9 & 1650 & & & & \\
\hline & & 81 & 3600 & & & & \\
\hline NGC 5347 & WHT 00 & 310 & 3600,1800 & & & & \\
\hline \multirow[t]{4}{*}{ NGC 5728} & LC 896 & 0 & 2400 & & & & \\
\hline & & 35 & 2400 & & & & \\
\hline & & 90 & 3600 & & & & \\
\hline & & 150 & 3600 & & & & \\
\hline \multirow[t]{8}{*}{ ESO 139-12 } & LC 596 & 35 & 3600 & & & & \\
\hline & & 65 & 3600 & & & & \\
\hline & & 125 & 3600 & & & & \\
\hline & LC 896 & 0 & 3600 & & & & \\
\hline & & 30 & 3600 & & & & \\
\hline & & 60 & 2400 & & & & \\
\hline & & 120 & 3600 & & & & \\
\hline & LC 898 & 5 & 2400 & & & & \\
\hline NGC 6814 & WHT 96 & 30 & 1800,1800 & & & & \\
\hline & & 120 & 1800,3600 & & & & \\
\hline & WHT 00 & 60 & 1800,1800 & & & & \\
\hline & LC 896 & 29 & 3600 & & & & \\
\hline & & 119 & 2400 & & & & \\
\hline & LC 898 & 20 & 2400 & & & & \\
\hline NGC 6860 & LC 896 & 4 & 3600 & & & & \\
\hline & & 34 & 3600 & & & & \\
\hline & & 64 & 3600 & & & & \\
\hline & & 124 & 2400 & & & & \\
\hline & LC 898 & 16 & 2400 & & & & \\
\hline NGC 6890 & LC 896 & 2 & 2400 & & & & \\
\hline & & 62 & 3600 & & & & \\
\hline & & 122 & 2400 & & & & \\
\hline & & 152 & 3600 & & & & \\
\hline & LC 898 & 13 & 2400 & & & & \\
\hline
\end{tabular}

For the WHT and CAHA runs the two exposure times correspond to $\mathrm{H} \beta$ and $\mathrm{H} \alpha$ respectively, the exposure time for the CaT being the sum of the two. The CaT WHT00 data for NGC 6814 and all the CaT CAHA00 data did not have sufficient signal to noise ratio to allow us to derive the stellar velocity distributions. 
I. Márquez et al.: Long slit spectroscopy of spirals with and without AGN, Online Material p 3

UGC 1395
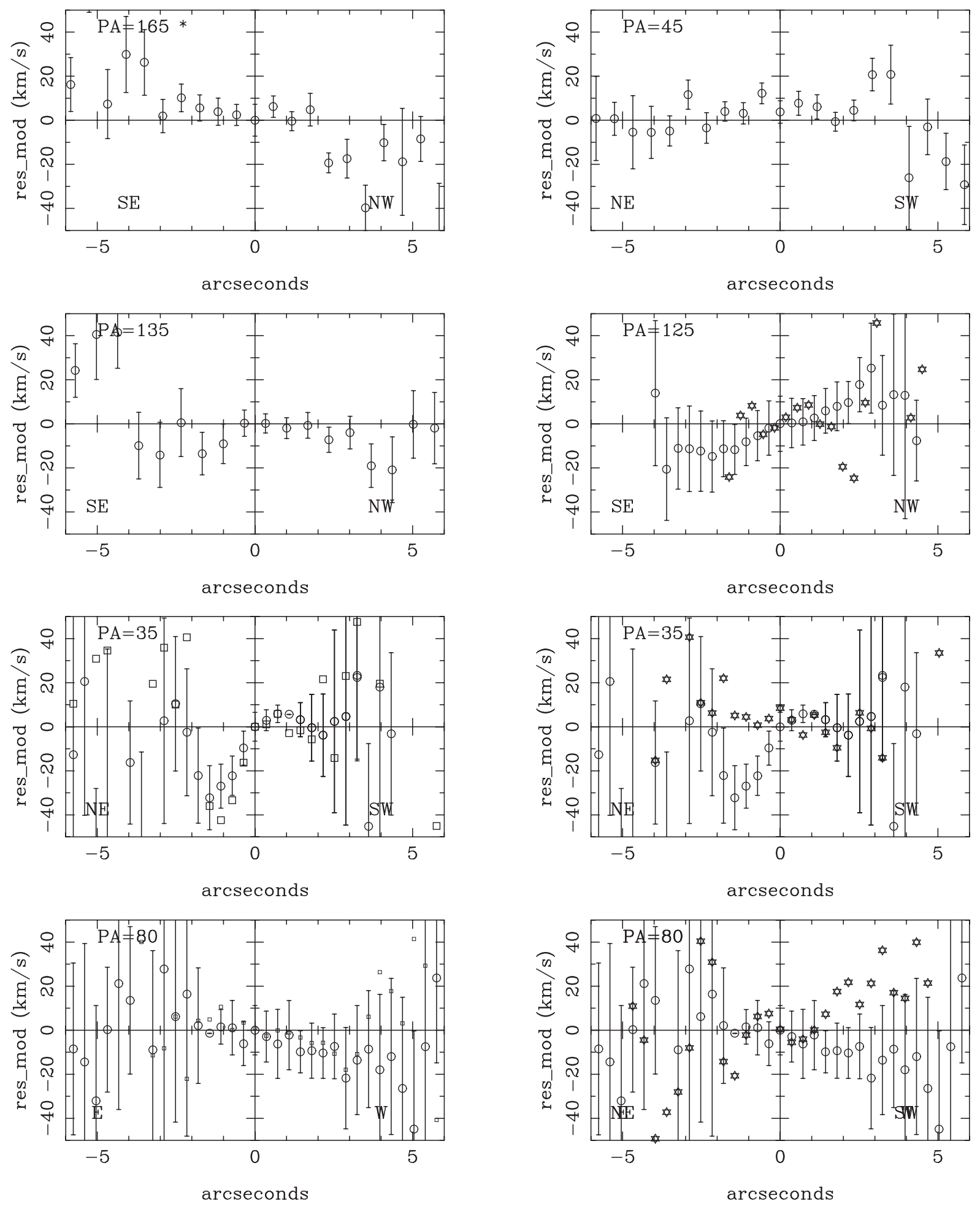

Fig. 3. Residuals from the model for UGC 1395. 
I. Márquez et al.: Long slit spectroscopy of spirals with and without AGN, Online Material p 4 IC 184
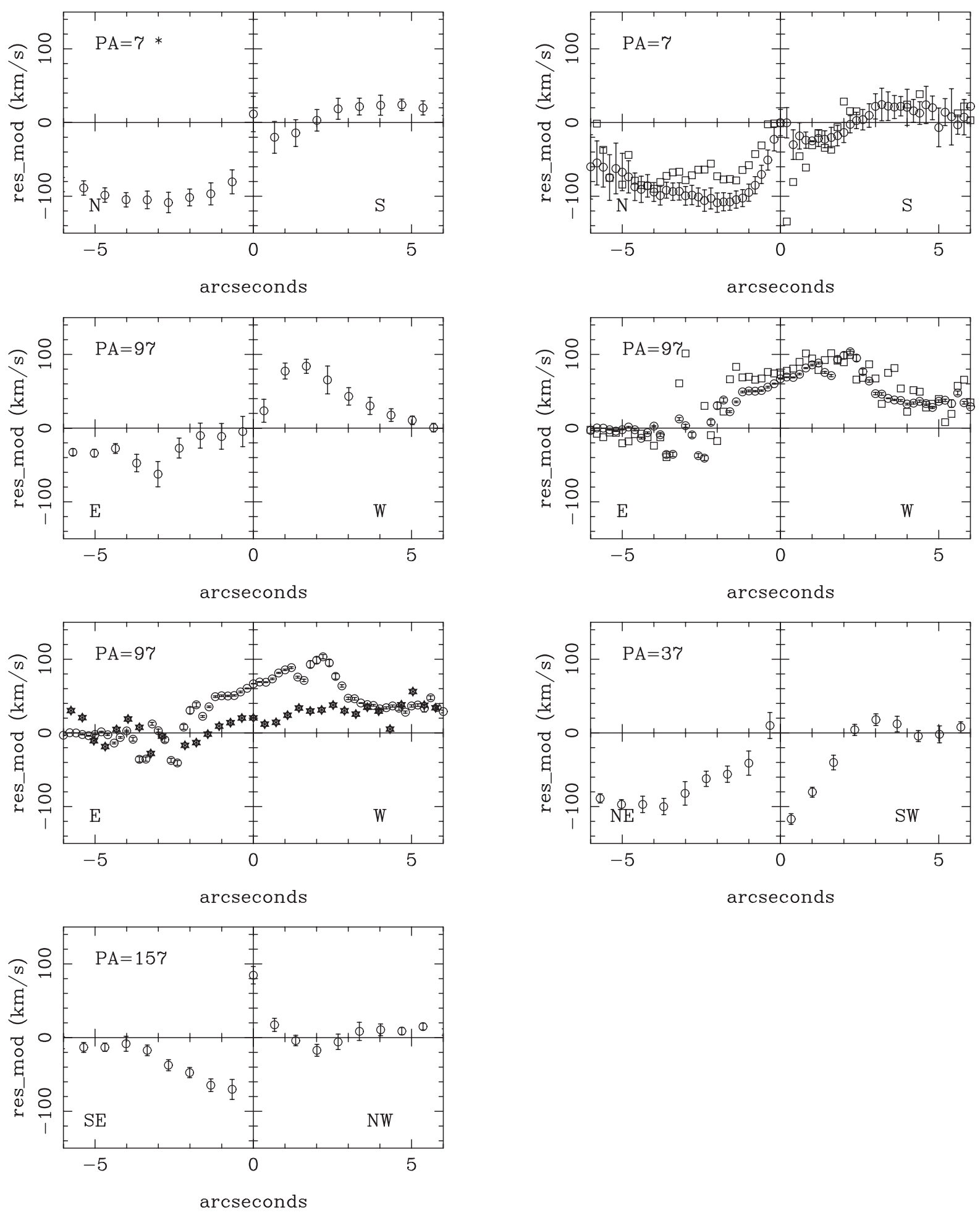

Fig. 5. Residuals from the model for IC 184 . 
I. Márquez et al.: Long slit spectroscopy of spirals with and without AGN, Online Material p 5

IC 1816
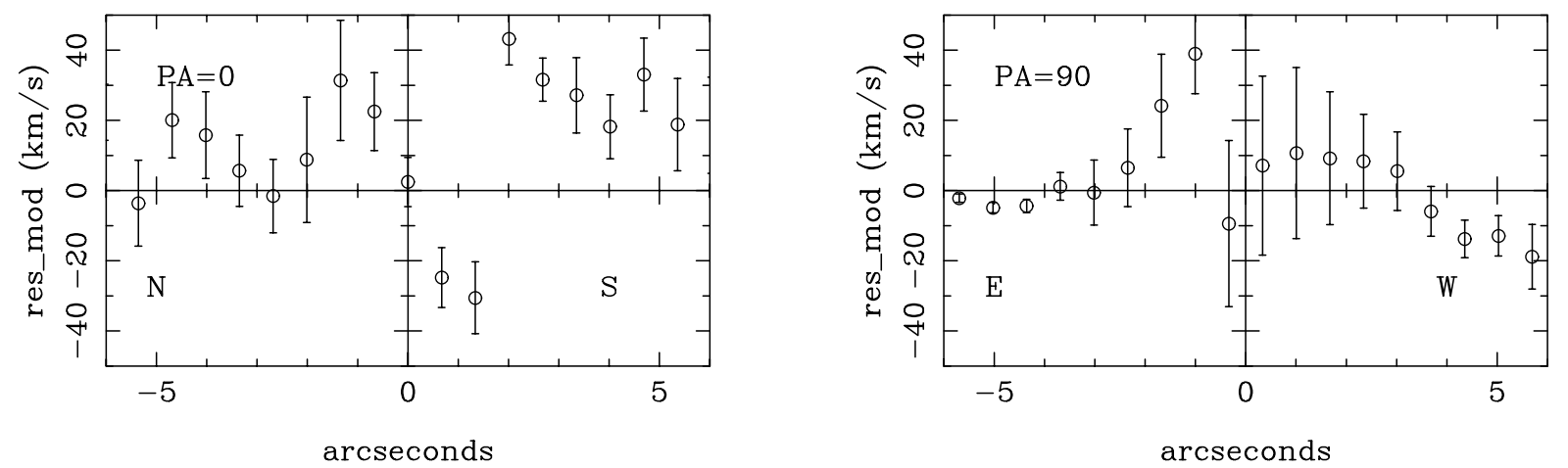

Fig. 7. Residuals from the model for IC 1816. 
I. Márquez et al.: Long slit spectroscopy of spirals with and without AGN, Online Material p 6

UGC 3223
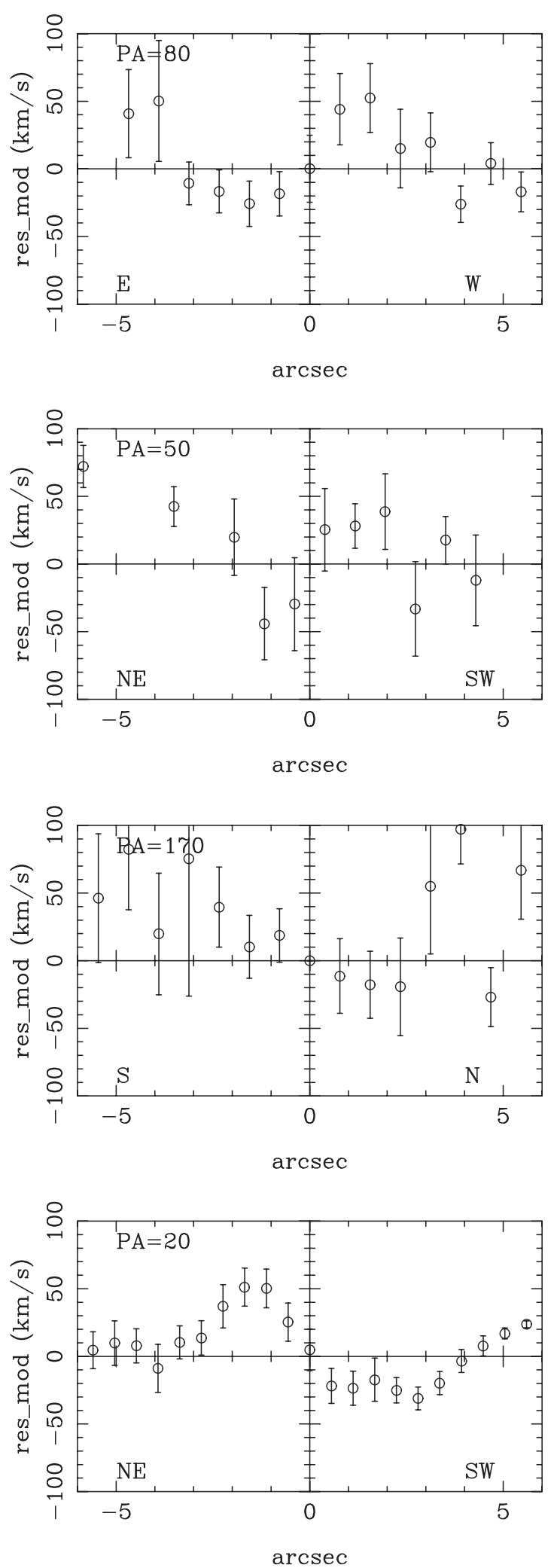

Fig. 9. Residuals from the model for UGC 3223.

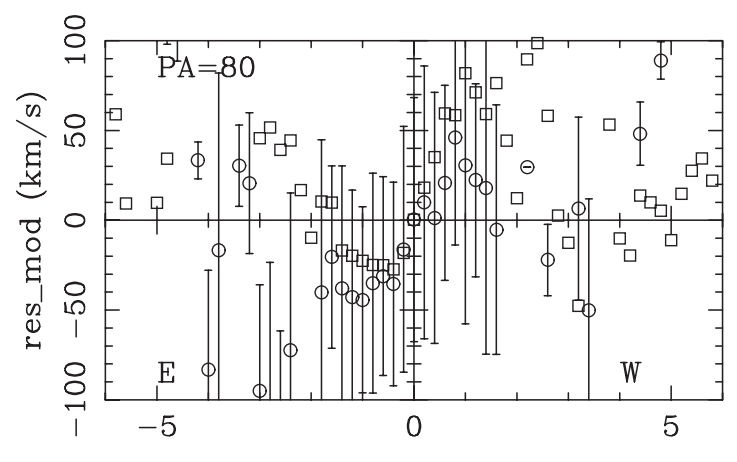

$\operatorname{arcsec}$
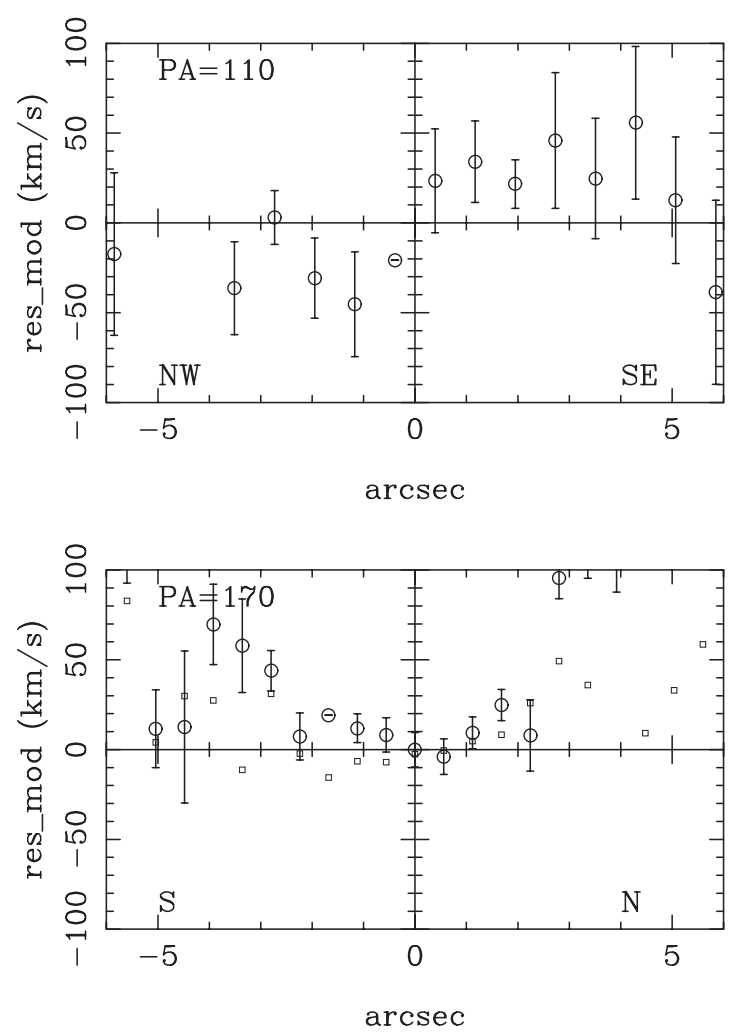
I. Márquez et al.: Long slit spectroscopy of spirals with and without AGN, Online Material p 7

NGC 2639
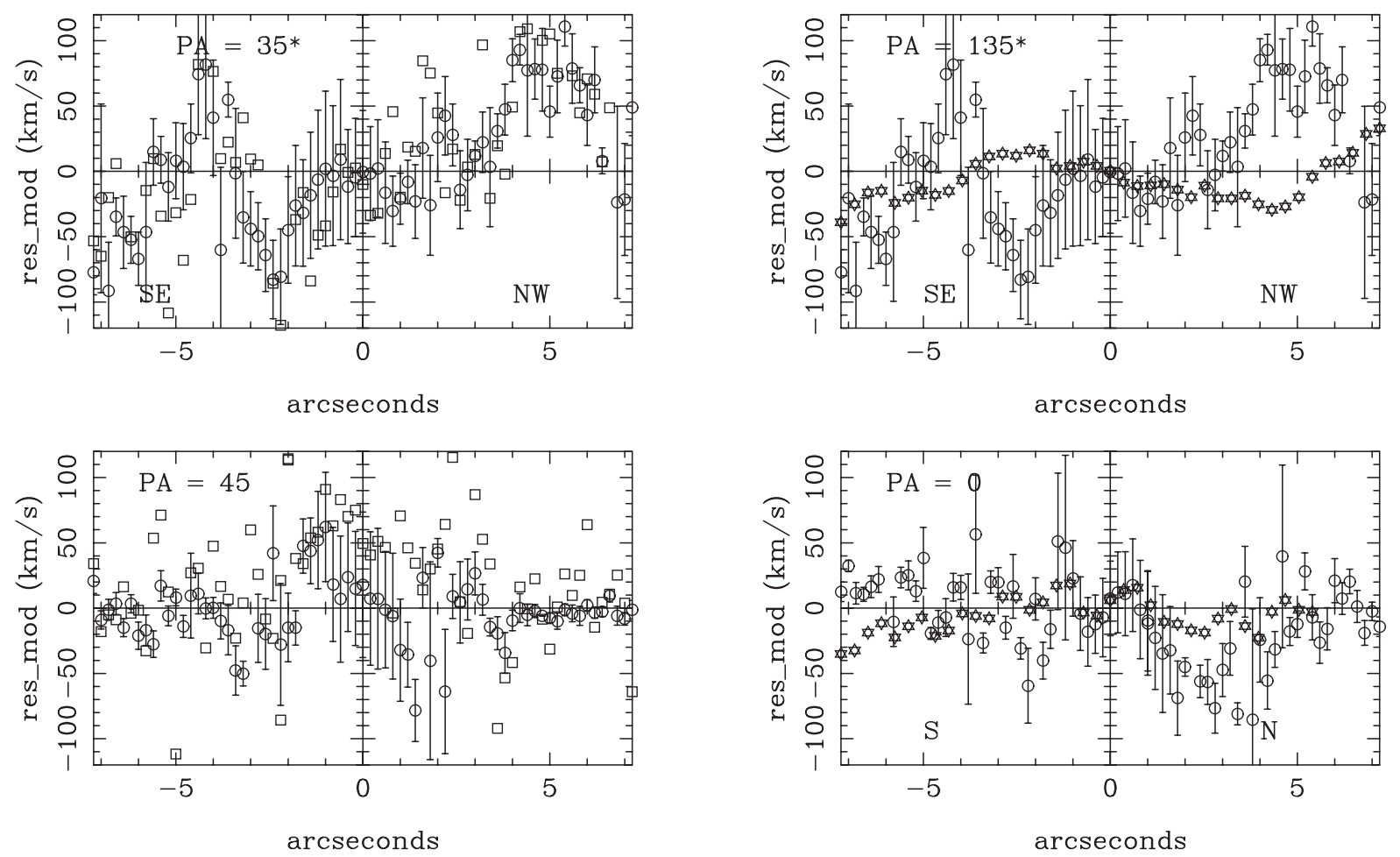

Fig. 11. Residuals from the model for NGC 2639.

IC 2510
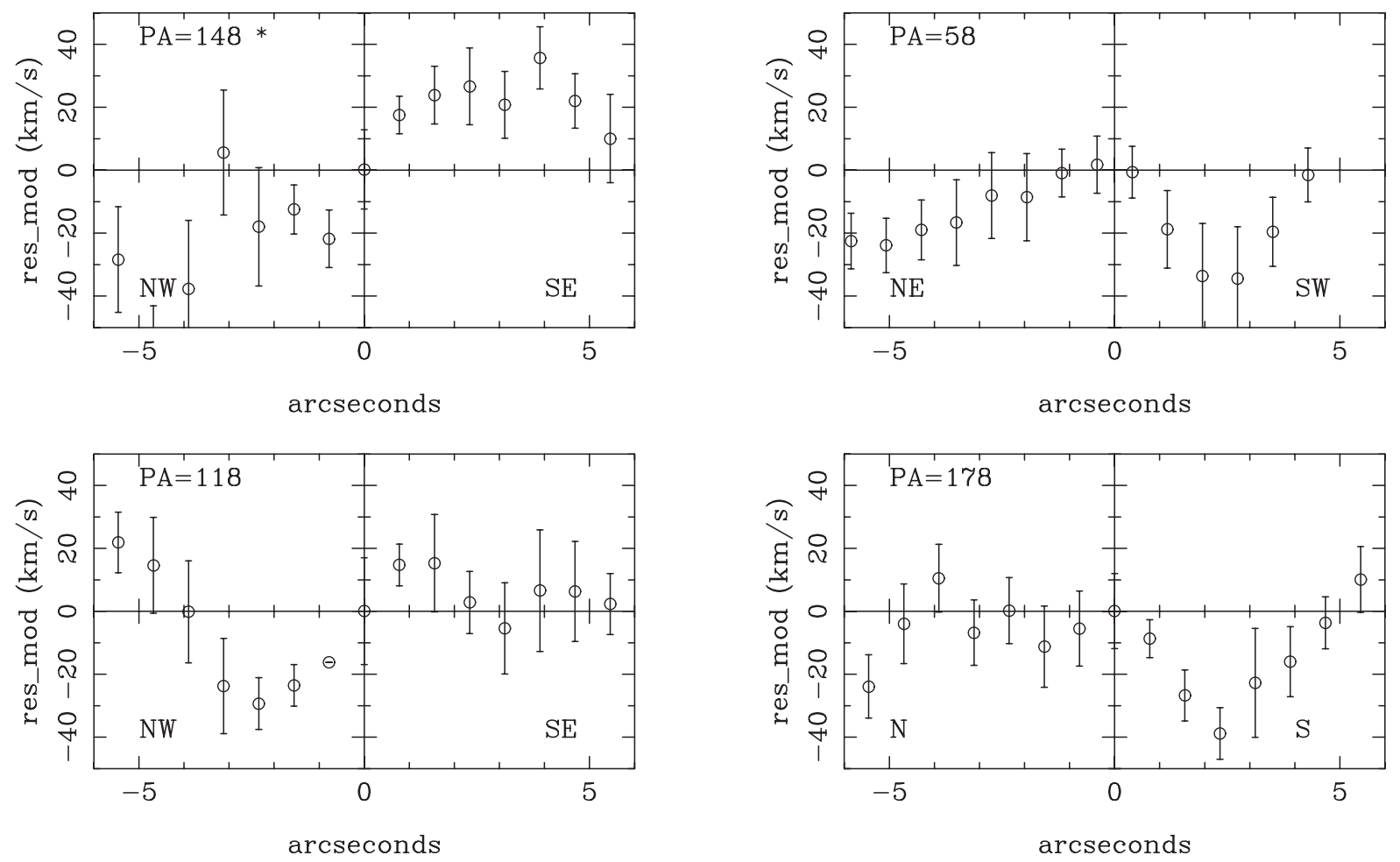

Fig. 13. Residuals from the model for IC 2510. 
I. Márquez et al.: Long slit spectroscopy of spirals with and without AGN, Online Material $p 8$

NGC 3660
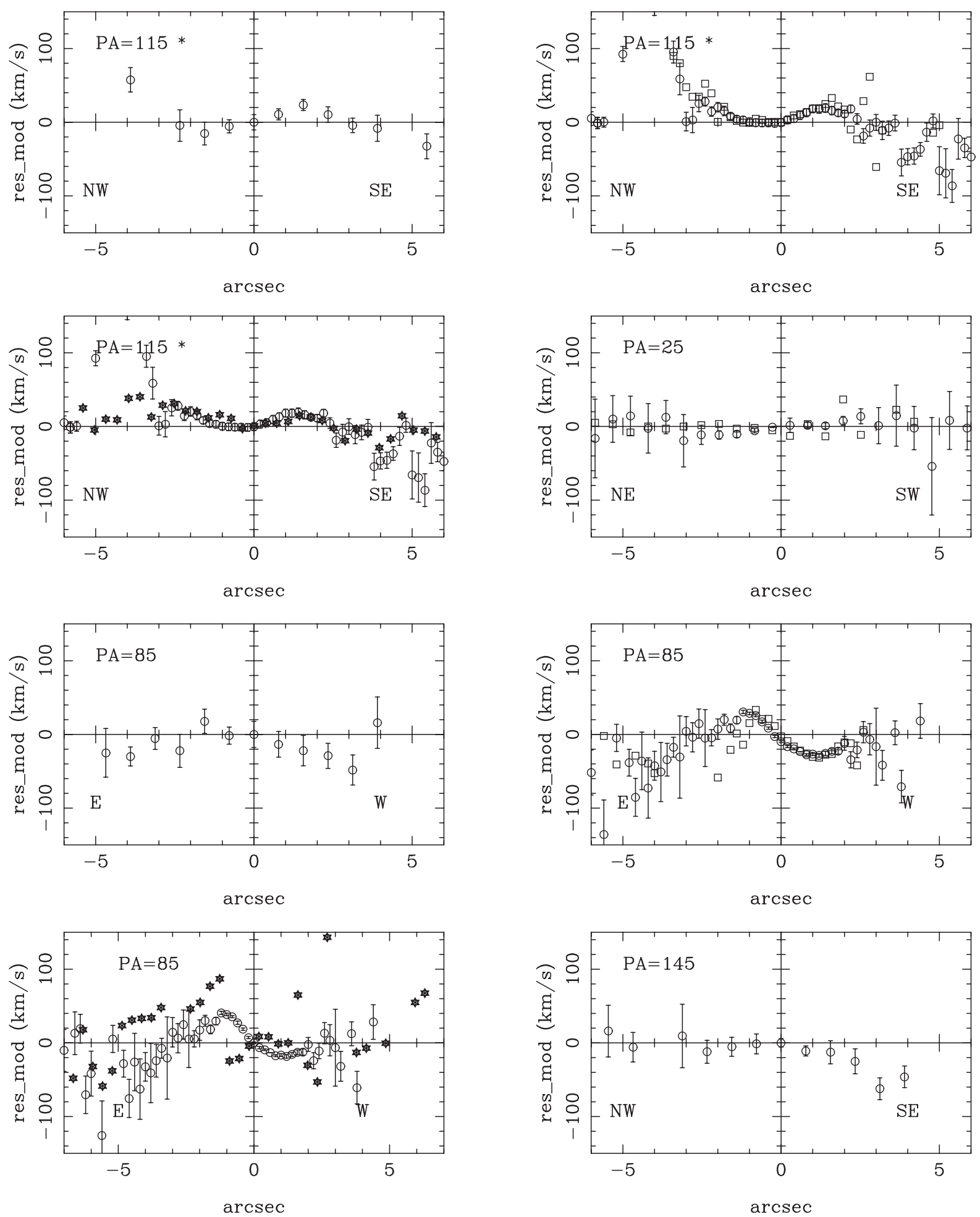

Fig. 15. Residuals from the model for NGC 3660. 
I. Márquez et al.: Long slit spectroscopy of spirals with and without AGN, Online Material p 9

NGC 4507

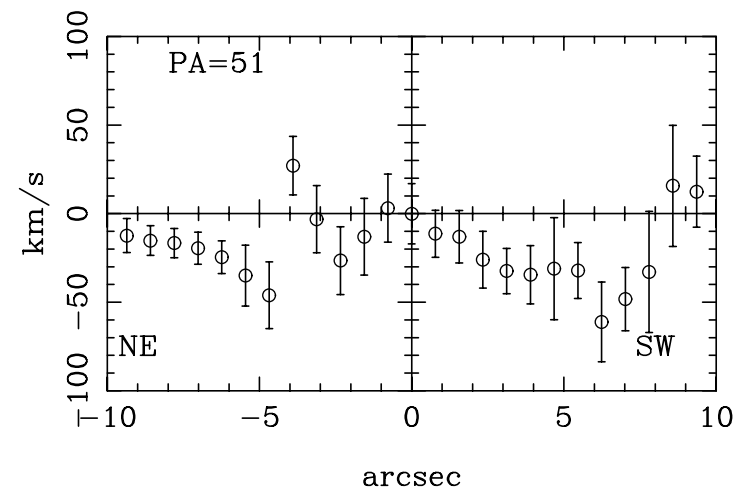

Fig. 17. Residuals from the model for NGC 4507.

NGC 4785

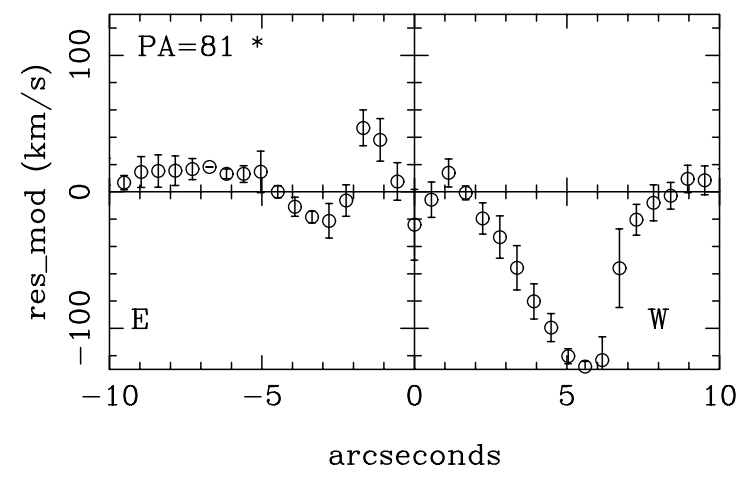

Fig. 19. Residuals from the model for NGC 4785.

NGC 5347

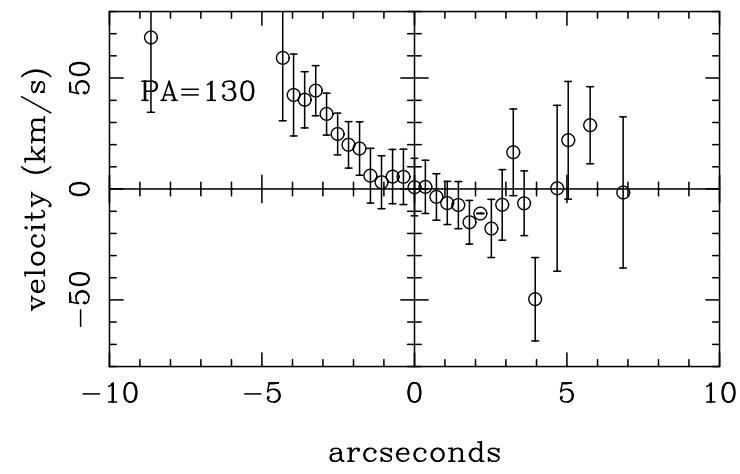

Fig. 21. Residuals from the model for NGC 5347.
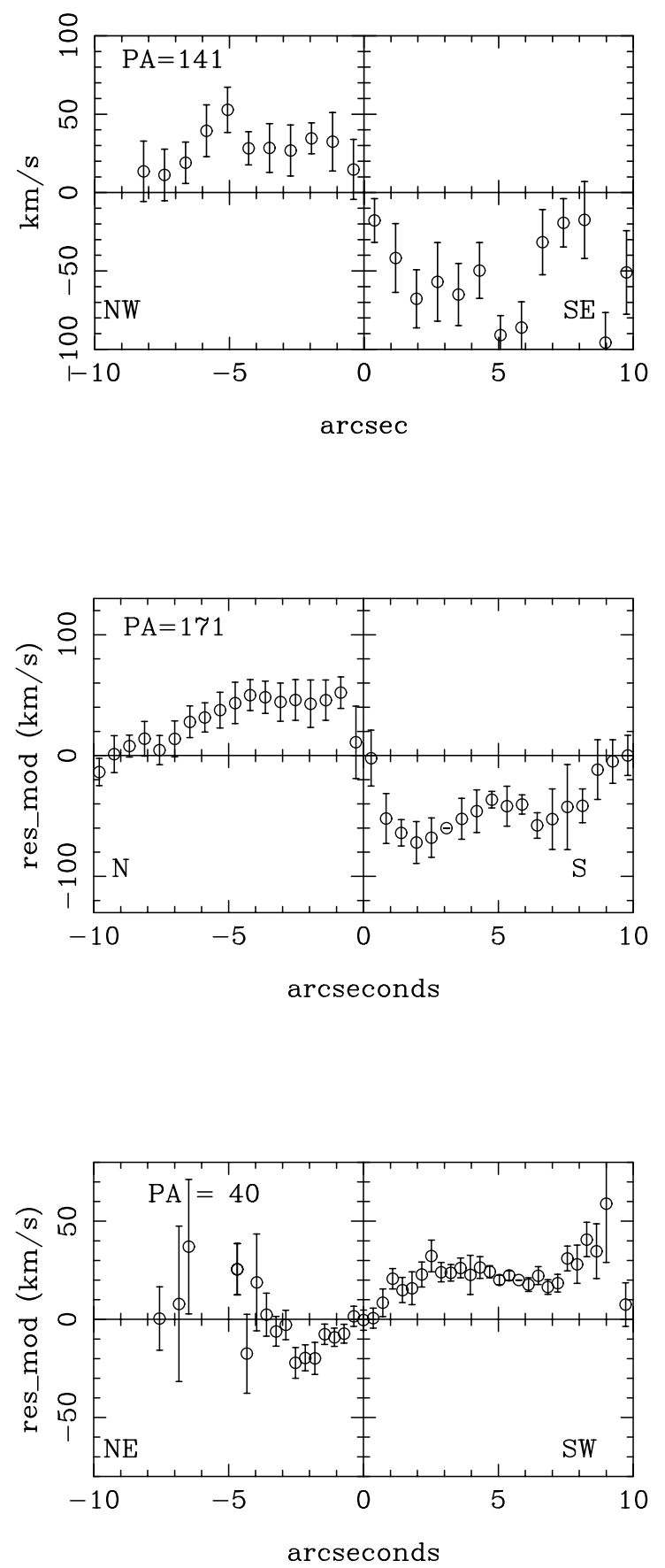
I. Márquez et al.: Long slit spectroscopy of spirals with and without AGN, Online Material p 10

NGC 5728
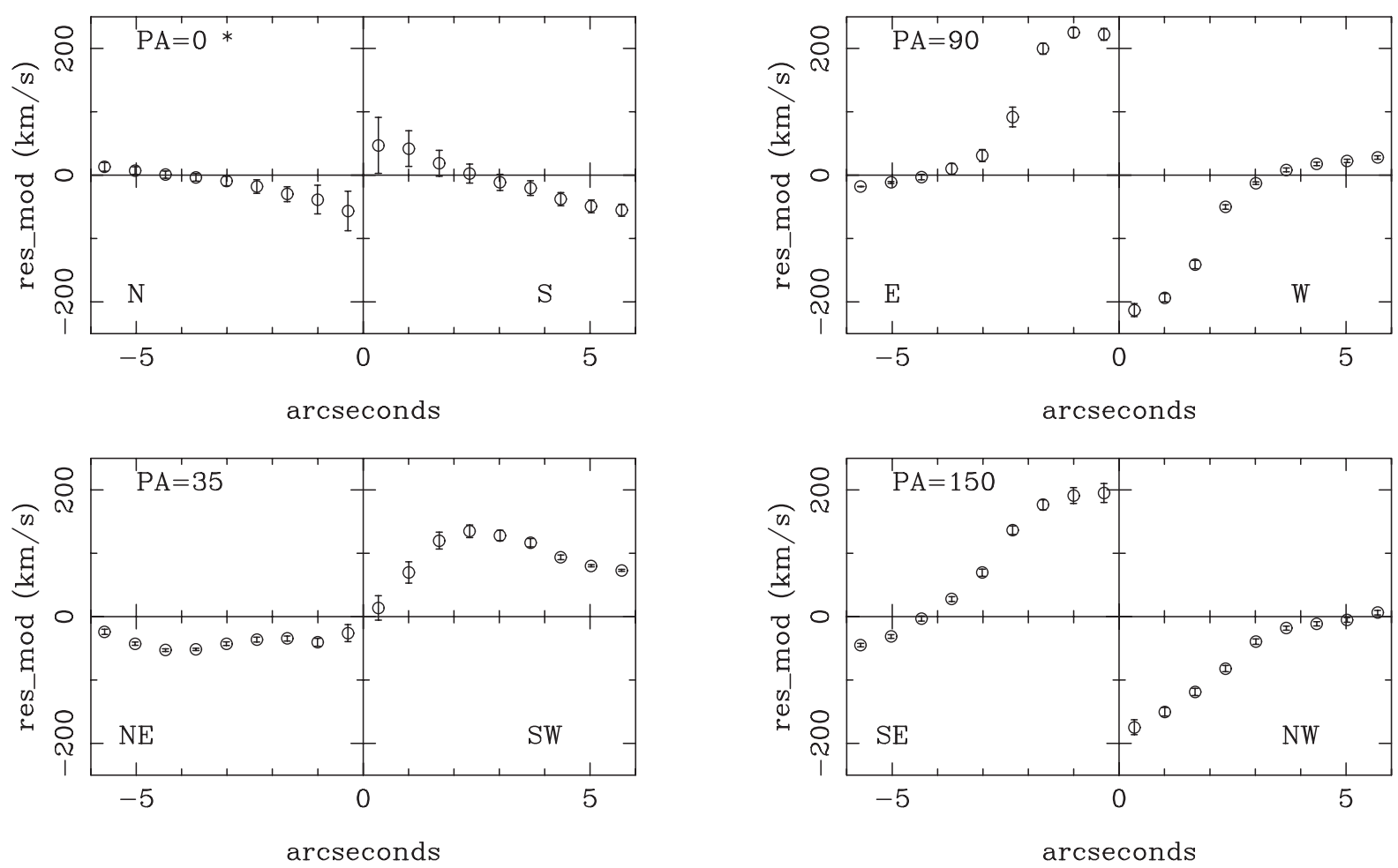

Fig. 23. Residuals from the model for NGC 5728. 
I. Márquez et al.: Long slit spectroscopy of spirals with and without AGN, Online Material $p 11$

ESO 139
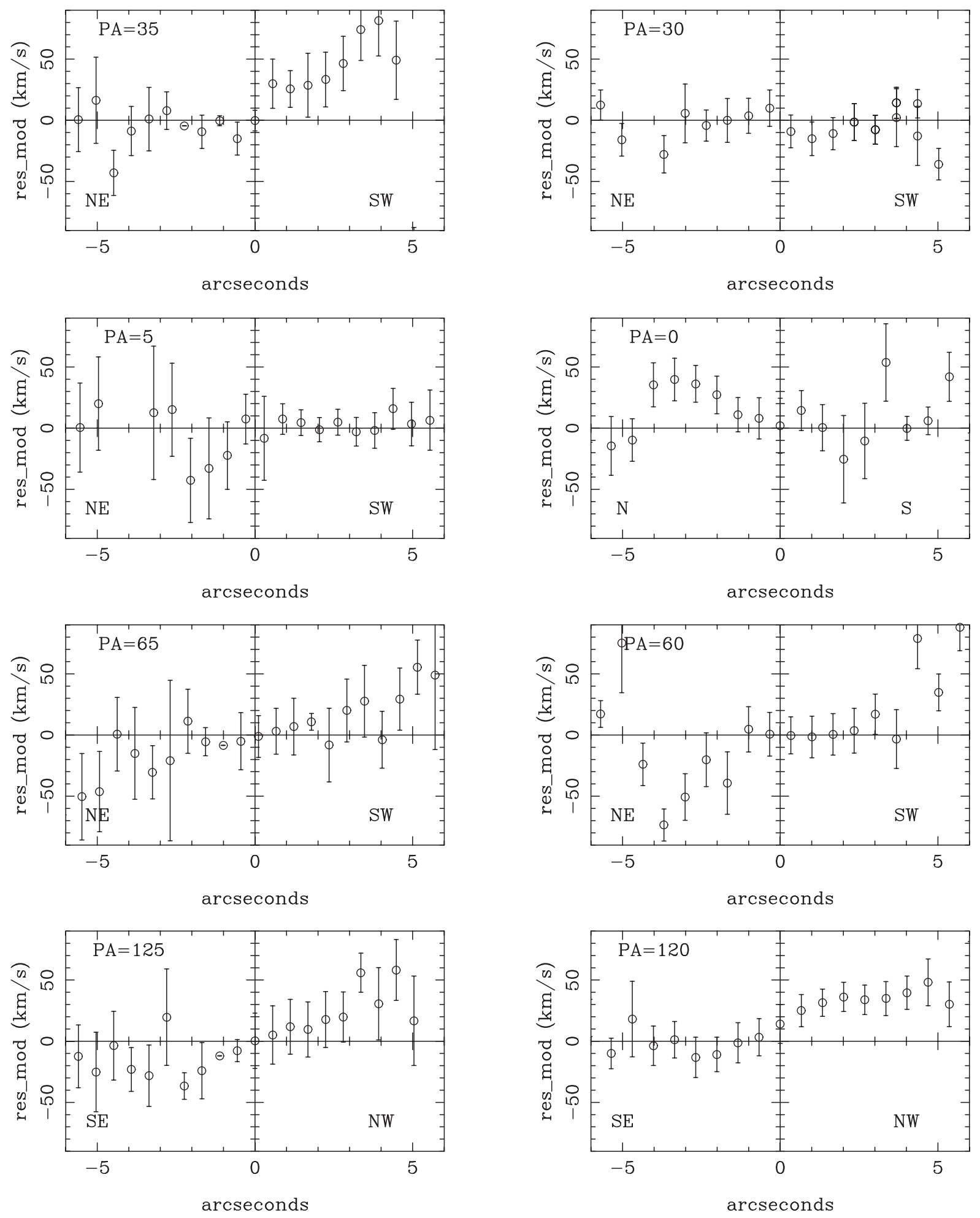

Fig. 25. Residuals from the model for ESO 139-G12. 
I. Márquez et al.: Long slit spectroscopy of spirals with and without AGN, Online Material p 12

NGC 6860
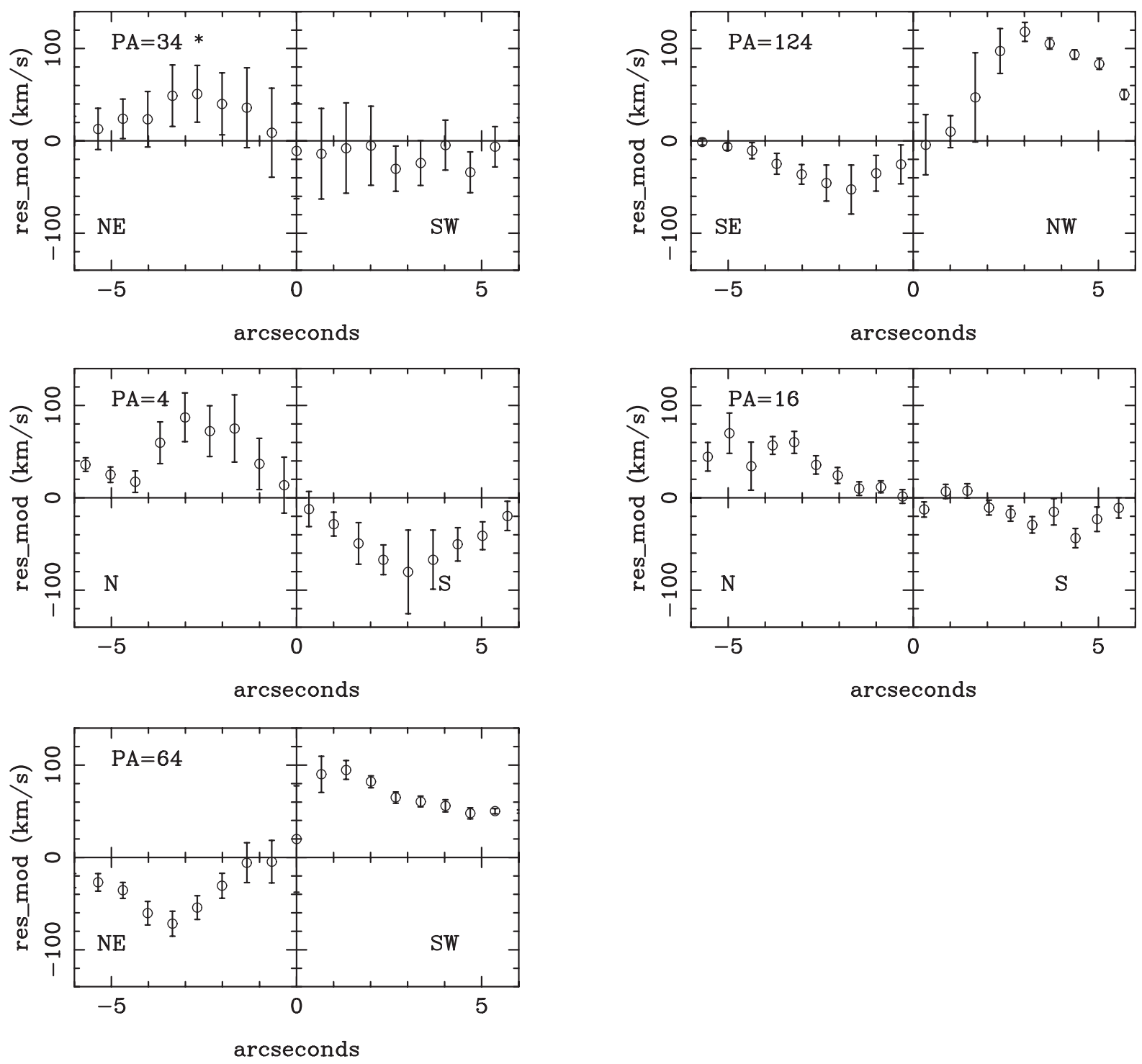

Fig. 28. Residuals from the model for NGC 6860. 
I. Márquez et al.: Long slit spectroscopy of spirals with and without AGN, Online Material p 13

NGC 6890
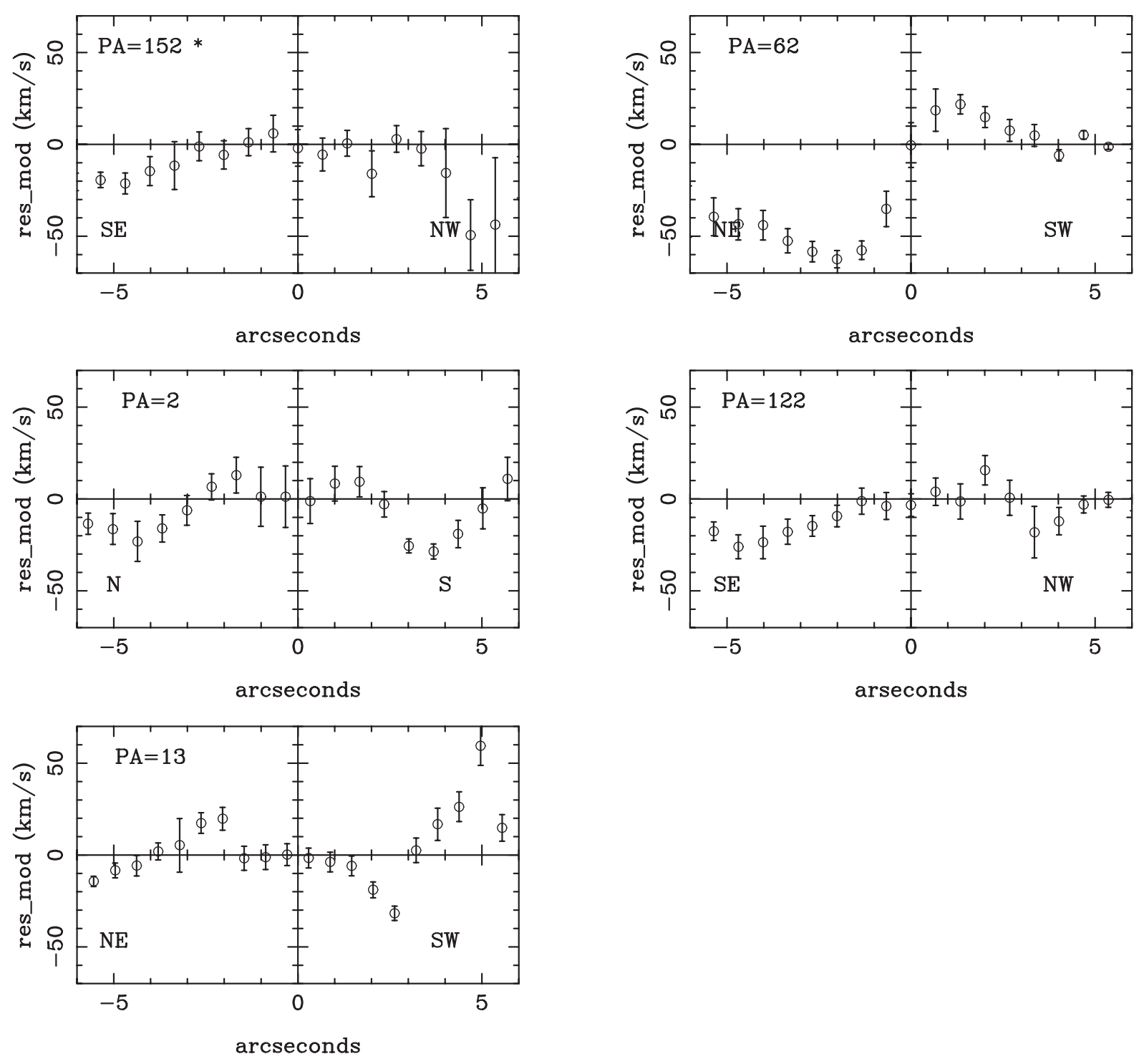

Fig. 30. Residuals from the model for NGC 6890. 
I. Márquez et al.: Long slit spectroscopy of spirals with and without AGN, Online Material p 14 NGC 151
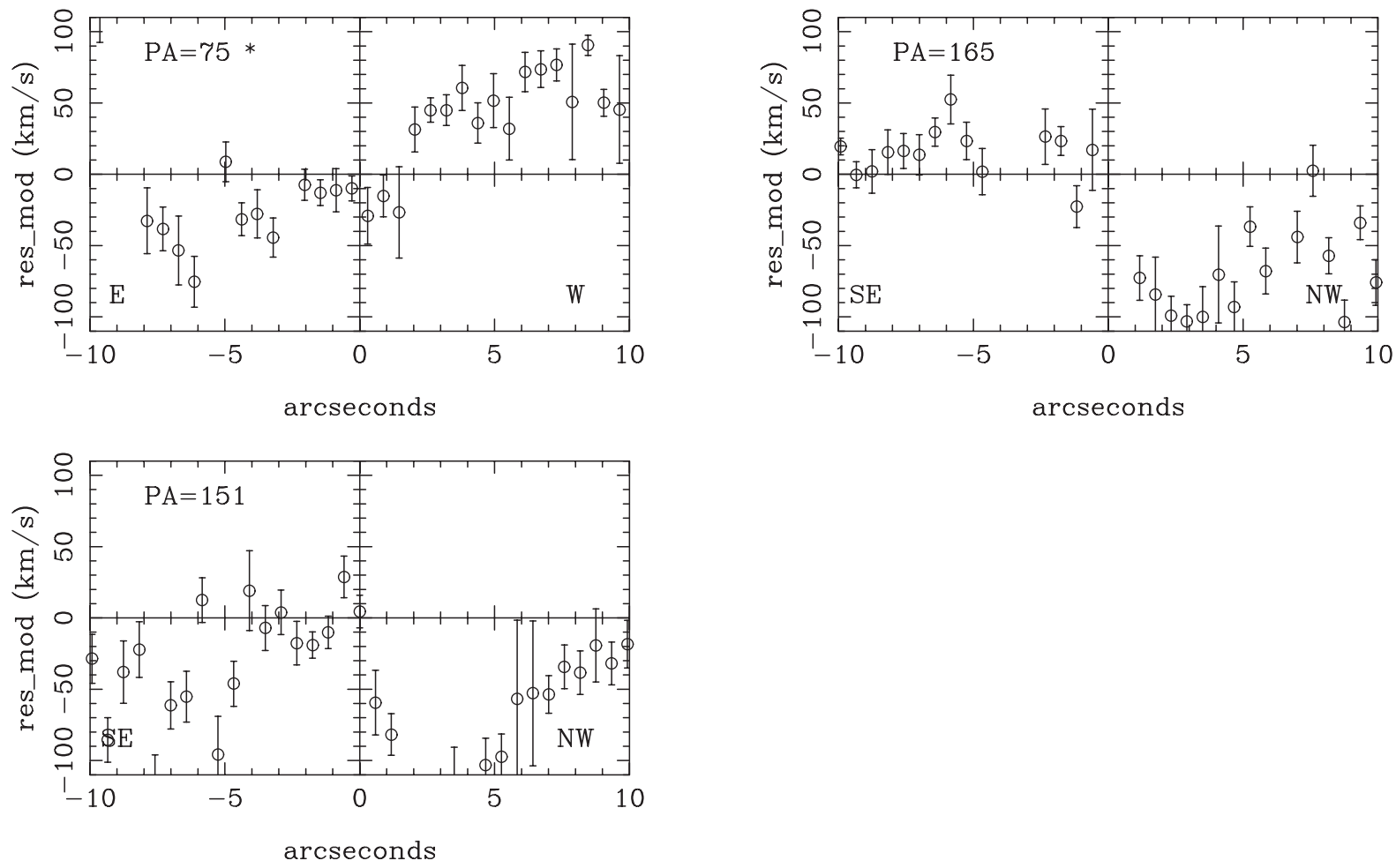

Fig. 32. Residuals from the model for NGC 151.

NGC 1357

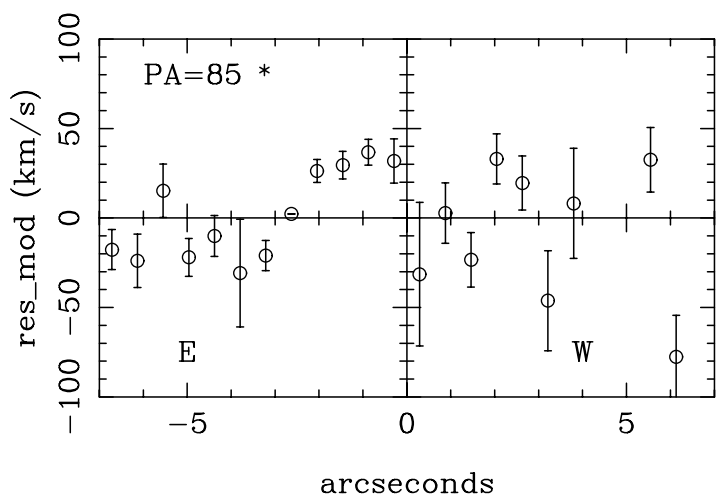

Fig. 34. Residuals from the model for NGC 1357. 
I. Márquez et al.: Long slit spectroscopy of spirals with and without AGN, Online Material p 15 IC 454
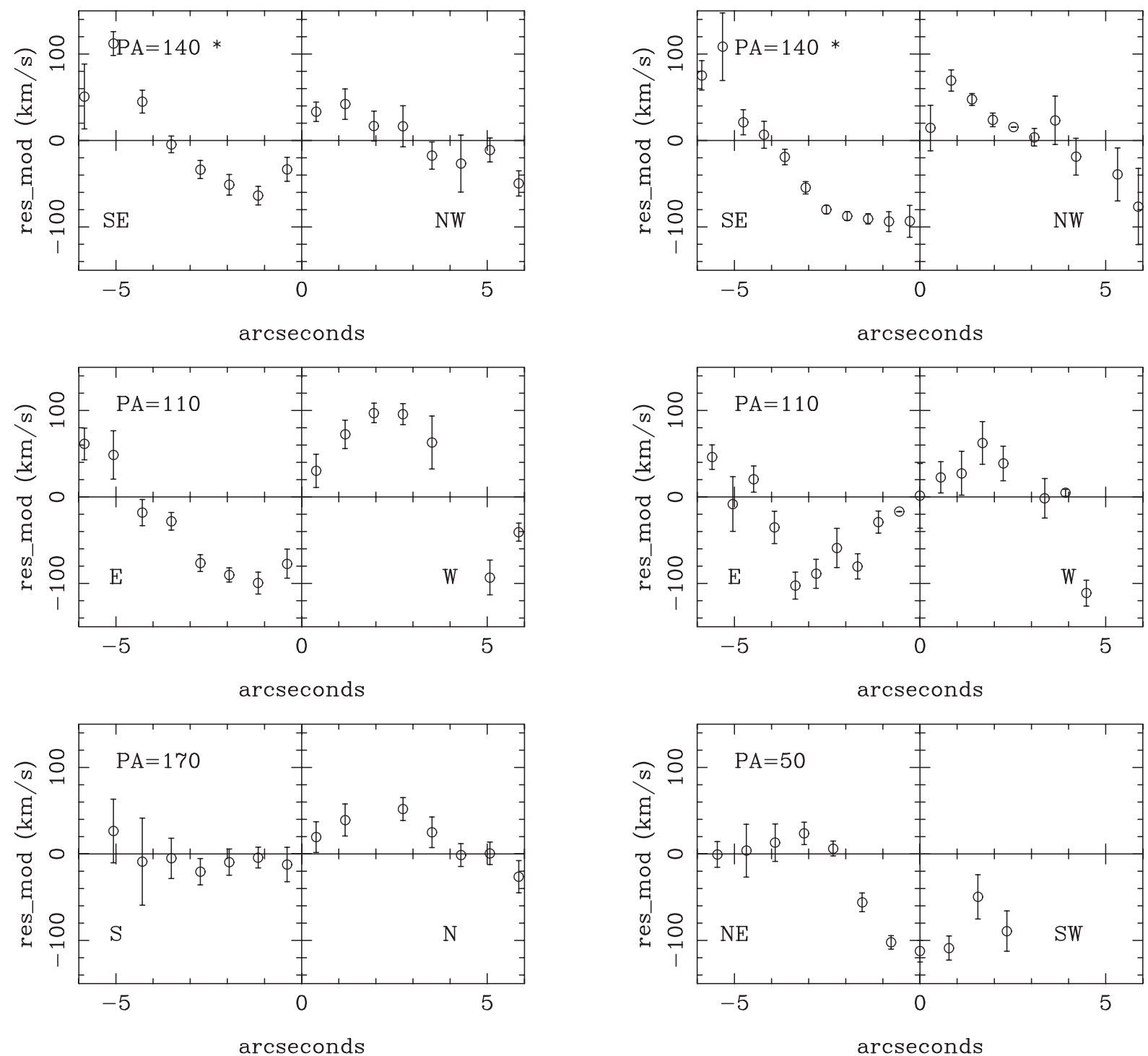

Fig. 36. Residuals from the model for IC 454.

NGC 3571

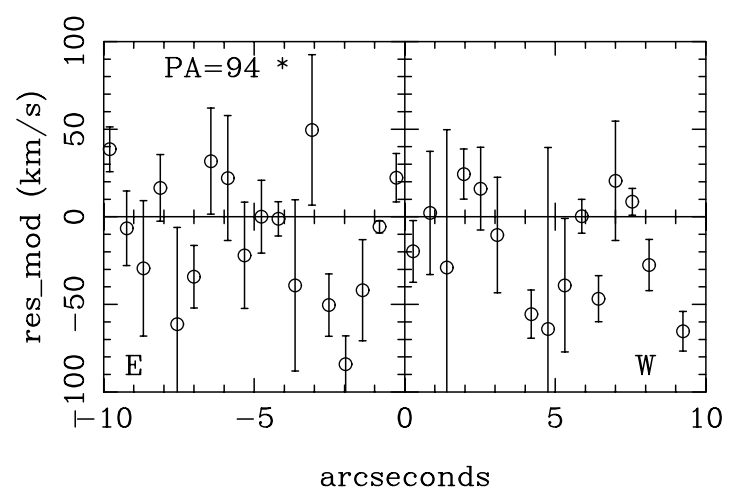

Fig. 38. Residuals from the model for NGC 3571. 
I. Márquez et al.: Long slit spectroscopy of spirals with and without AGN, Online Material p 16

NGC 6012
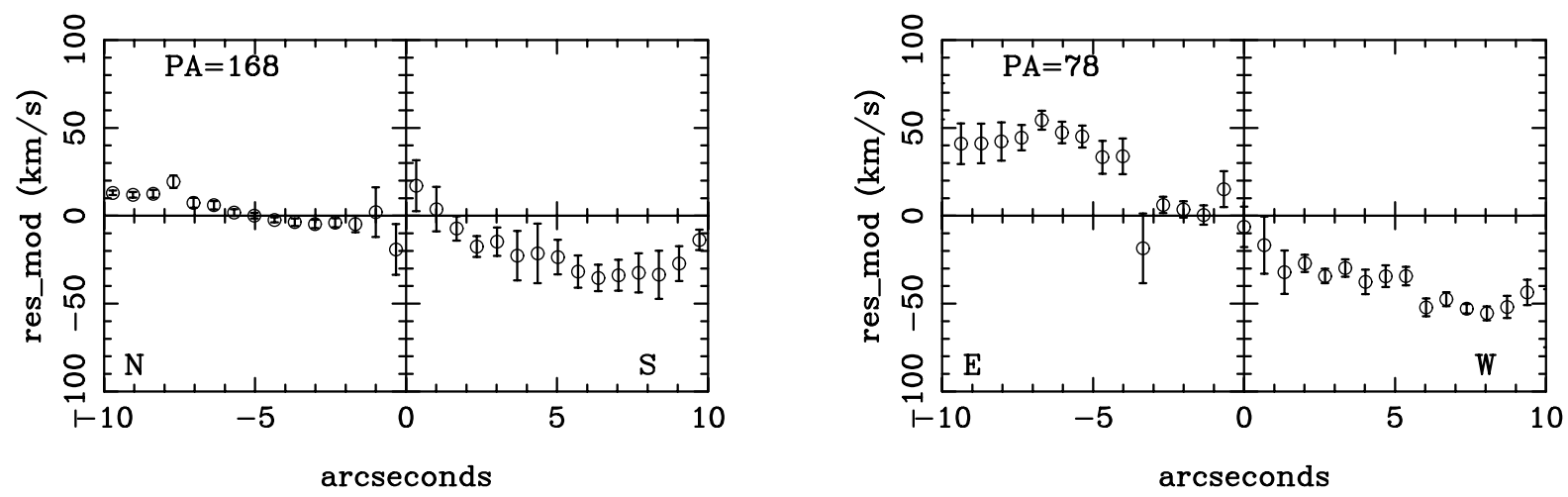

Fig. 40. Residuals from the model for NGC 6012.

NGC 7328
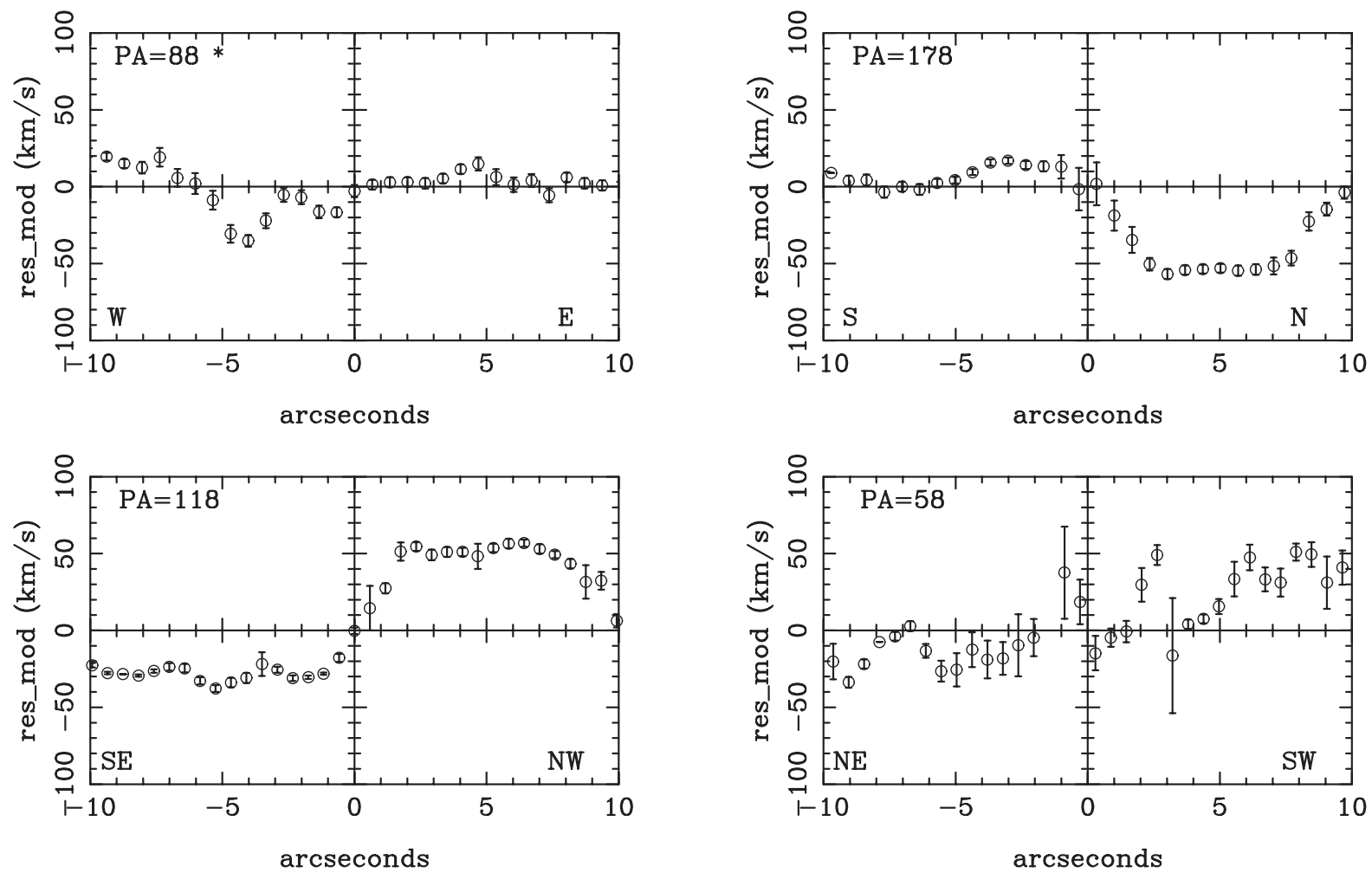

Fig. 42. Residuals from the model for NGC 7328. 
I. Márquez et al.: Long slit spectroscopy of spirals with and without AGN, Online Material p 17

NGC 2712

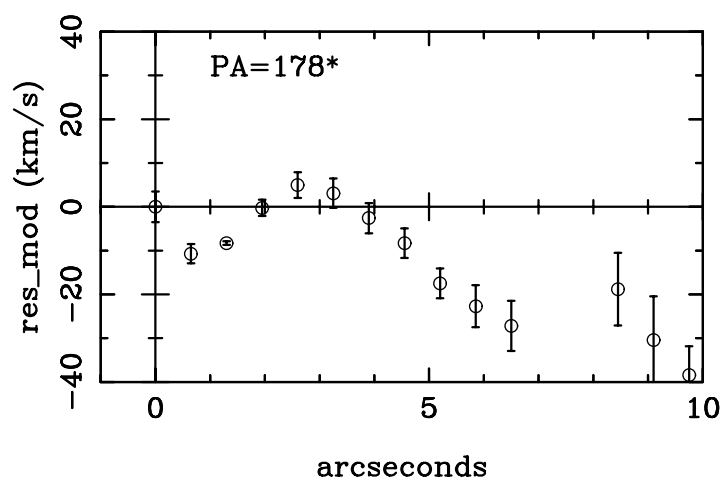

Fig. 44. Residuals from the model for NGC 2712.

NGC 3835

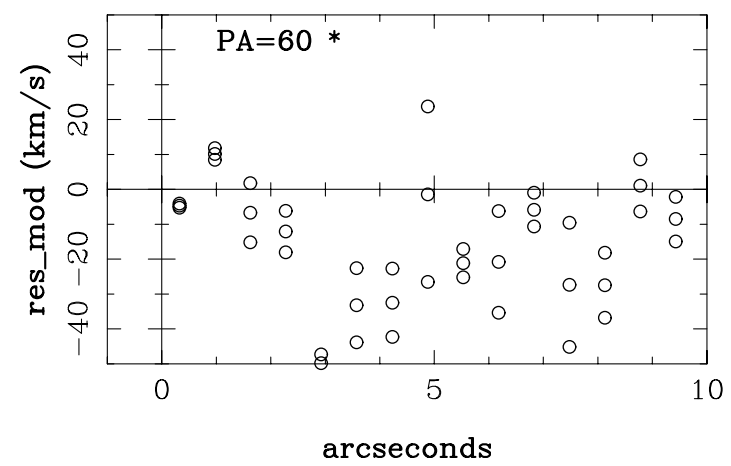

Fig. 46. Residuals from the model for NGC 3835.

NGC 4162

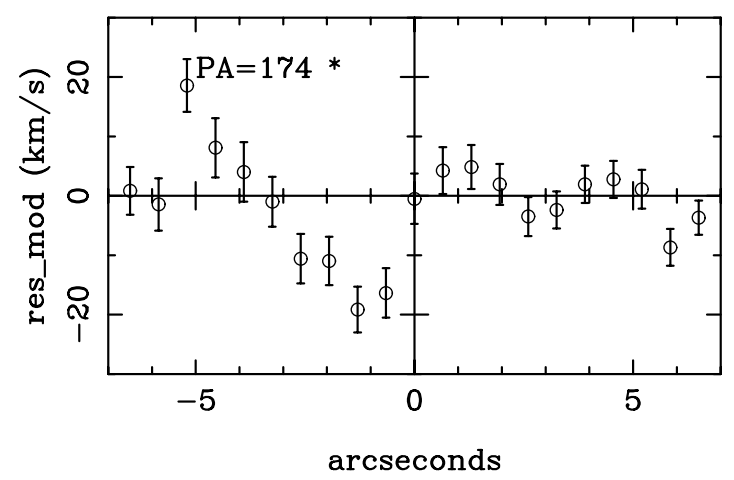

Fig. 48. Residuals from the model for NGC 4162.
NGC 4290

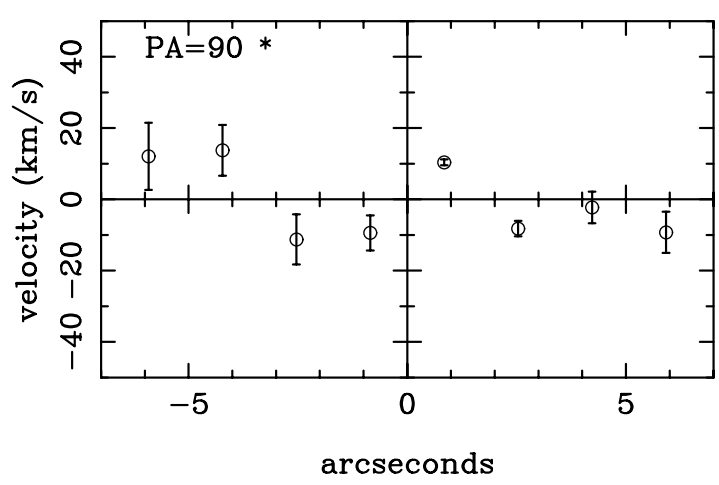

Fig. 50. Residuals from the model for NGC 4290.

NGC 6155

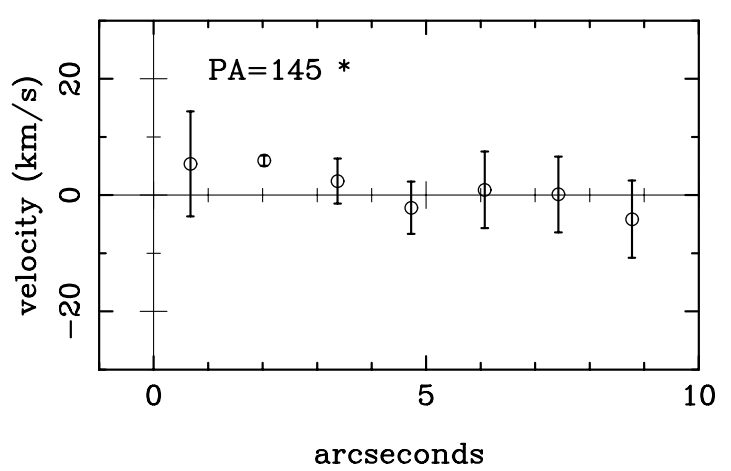

Fig. 52. Residuals from the model for NGC 6155. 